\title{
Ultrasonic Signal Processing for Continuous Measurements of Tissue Displacement and Thickness during Muscle Contraction
}

$$
\text { By }
$$

\section{Hisham Turkistani}

\author{
A thesis submitted to \\ The Faculty of Graduate Studies and Research \\ in partial fulfillment of the requirements for the degree of
}

\section{Master of Applied Science in Biomedical Engineering}

Ottawa-Carleton Institute of Biomedical Engineering (OCIBME)

Department of Systems and Computer Engineering

\author{
Carleton University \\ Ottawa Ontario, Canada
}

November 2013

Copyright (C) Hisham Turkistani, 2013 
The undersigned recommend to

The Faculty of Graduate Studies and Research

acceptance of the thesis

\section{Ultrasonic Signal Processing for Continuous Measurements of Tissue Displacement and Thickness during Muscle Contraction}

submitted by Hisham Turkistani

in partial fulfillment of the requirements for the degree of

Master of Applied Science in Biomedical Engineering

Yuu Ono

Thesis Supervisor

Roshdy H.M. Hafez

Chair, Department of System and Computer Engineering

Carleton University 


\begin{abstract}
Tissue displacement and thickness are useful parameters for quantifying muscle function. These parameters can be obtained using ultrasound with high frame rate and reasonable spatial resolution. However, a conventional hand-held ultrasonic probe that is bulky, rigid, and heavy may not be suitable for continuous muscle monitoring during physical activities. This research aimed to measure tissue displacement and thickness variation during muscle contraction using a wearable ultrasonic sensor. However, the energy of the transmitted ultrasonic waves using the wearable ultrasonic sensor is lower than that of a conventional probe. In order to overcome this issue, selected signal processing techniques were applied and compared. It was found in the numerical simulation experiments that the frequency-domain techniques, in particular LQ-factorization, had better tracking accuracy than the time-domain techniques. In the in-vivo experiment, ultrasonic signals were acquired at a forearm during isometric contraction. The tissue boundary displacements and thickness changes were successfully obtained.
\end{abstract}




\section{Statement of Originality}

The accompanying thesis submitted for the degree of M.A.Sc. in Biomedical Engineering at Carleton University is based on work conducted by the author. All the work recorded in this thesis is original unless otherwise acknowledged in the text or by references. Some of these results have been presented and appear in conference publication. A summary of the publication and the results of this research is provided below.

Hisham Turkistani, Ibrahim AlMohameed, and Yuu Ono "Continuous monitoring of muscle thickness changes during isometric contraction using a wearable ultrasonic sensor," Conference Proceeding of the Canadian Medical and Biological Engineering Society, Ottawa, Canada, May 21-24, 2013.

One of the signal processing techniques that used in this paper is the LQ-factorization which is mentioned in Chapter 3. Results of the experiment of the paper appear in Chapter 5 of this thesis. The tissues boundary displacement and thickness were obtained based on the LQ-factorization technique. The experiment was conducted using wearable ultrasonic sensor that was developed by our research group. This paper was presented as a poster presentation by the author at the 2013 CMBES/APIBQ joint conference on Canadian Medical and Biological Engineering Society in Ottawa, Canada on May ***, 2013. 


\section{Acknowledgments}

Foremost, I would like to express my sincere gratitude to my manager at Saudi Food \& Drug Authority, Eng. Abdullah AlRasheed, and to all personnel at Saudi Foods \& Drug Authority for giving me a scholarship and enabling me to pursue my dream.

I would like to acknowledge my supervisor Dr. Yuu Ono, Associate Professor of Systems and Computer Engineering, Carleton University, for his time, expertise, guidance, encouragement, support, and kindness. Besides my supervisor, I would also like to thank my colleagues at Carleton University Zhen Qu, Ibrahim AlMohameed, Andy Huang, Anwar Fallatah, Faisal Arafshah, and Justin Wagenaar. Without their knowledge and assistance this study would not have been successful.

My sincere thanks also go to the Saudi Arabian Cultural Bureau (SACB) who made my stay in Canada with my family affordable and enjoyable.

A special thanks to my wife Hanan Gabil, who made my efforts worthwhile by giving me the most precious gift, my daughter Reeman, during my work on this thesis. Also to my mother Maryam, my brothers, and all my relatives and friends, thank you for standing by my side when I needed you.

Finally, from my heart, I would like to dedicate this thesis to my father, Abdullah Turkistani, who gave me all his support in my educational life until the last day of his life. He would be so proud by my accomplishment. 


\section{Contents}

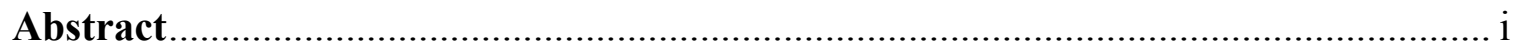

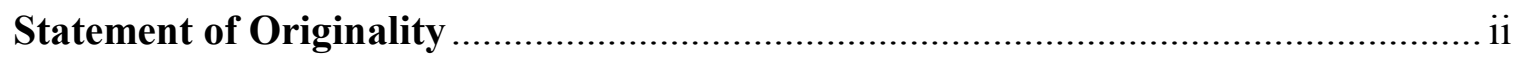

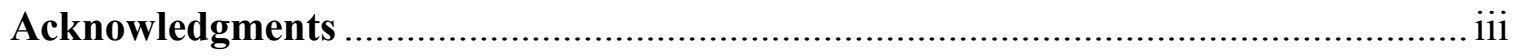

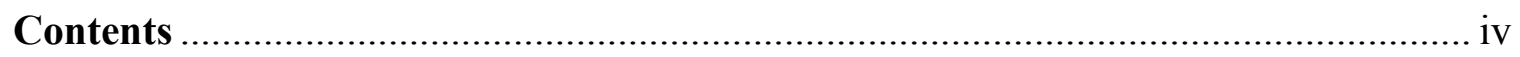

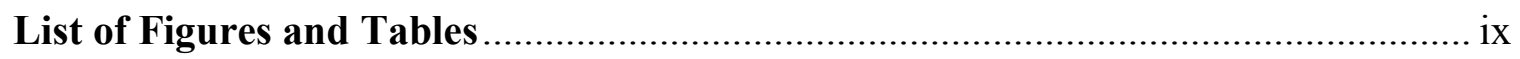

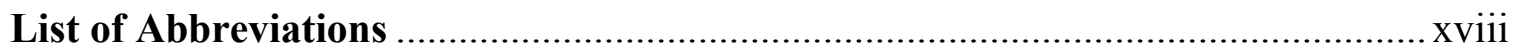

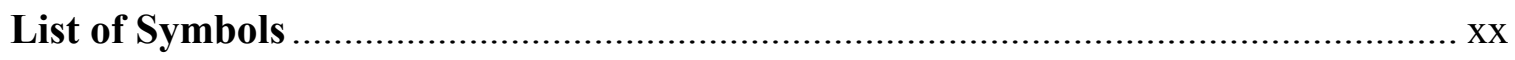

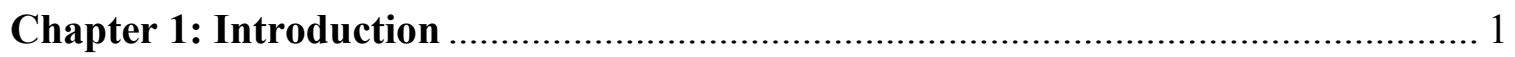

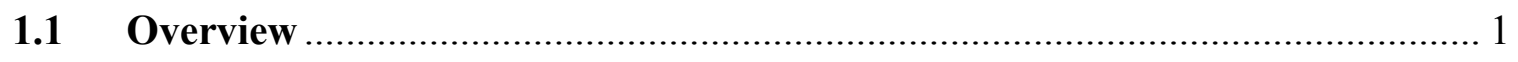

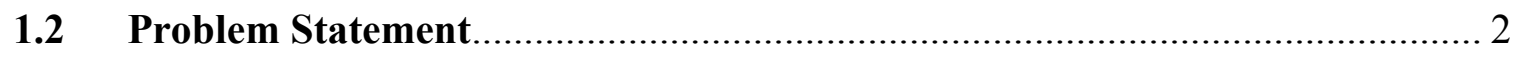

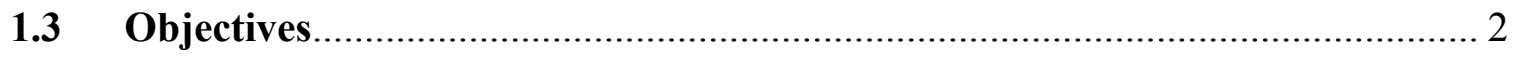

1.4 Thesis Contributions.............................................................................. 3

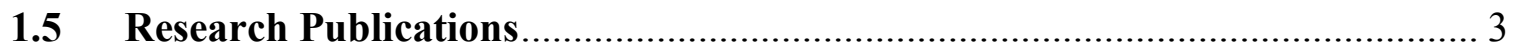

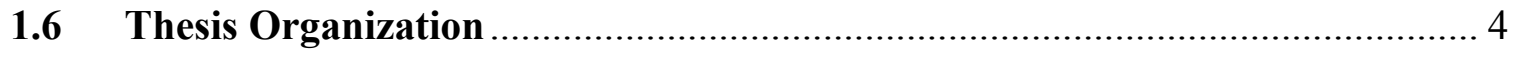

Chapter 2: Technical Background Review ...................................................... 5

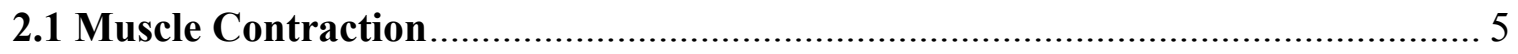




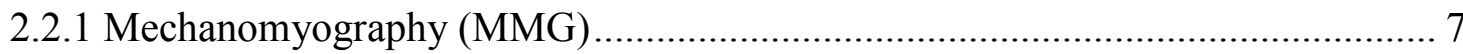

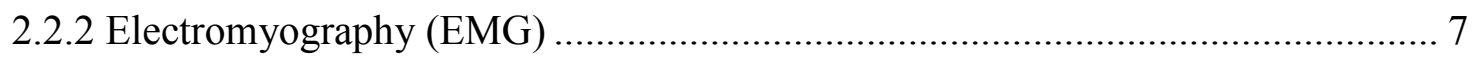

2.2.3 Magnetic Resonance Imaging (MRI)..................................................... 8

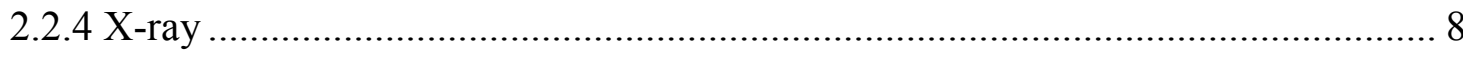

2.2.5 Computed Tomography $(\mathrm{CT})$................................................................. 9

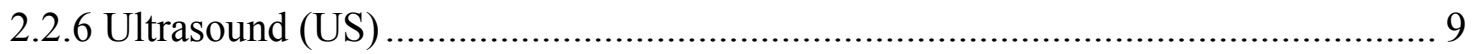

2.2.7 Current Research and Applications ............................................................ 10

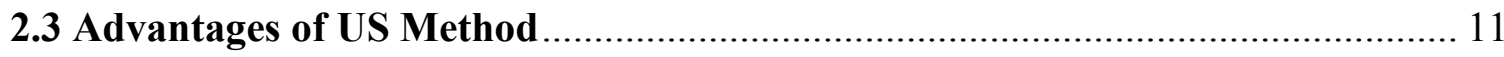

2.4 US for Muscle Displacement and Thickness Measurement ............................. 11

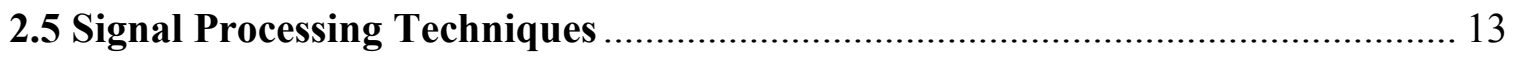

2.5.1 Echo Peak Detection .................................................................................... 13

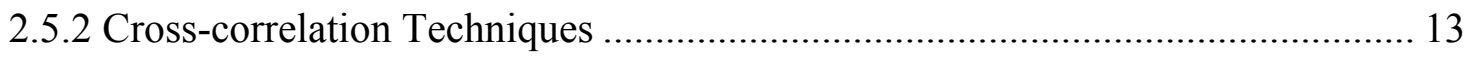

2.5.3 Phase Detection Techniques ........................................................................ 14

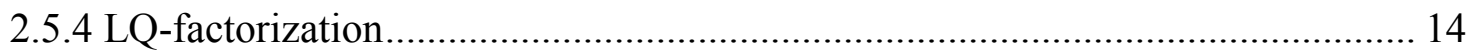

Chapter 3: Measurement Method of Tissue Displacement and Thickness .............. 15

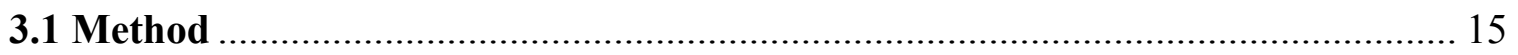

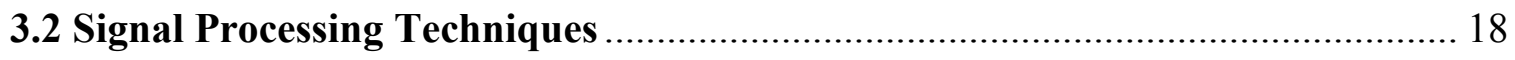

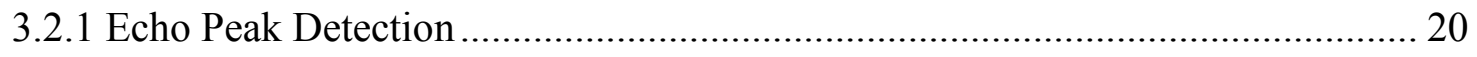




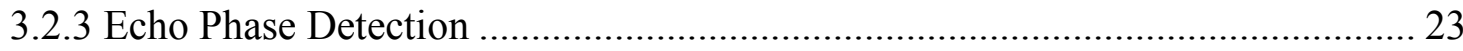

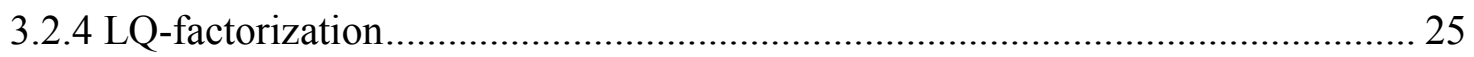

Chapter 4: Numerical Simulation Experiments............................................... 28

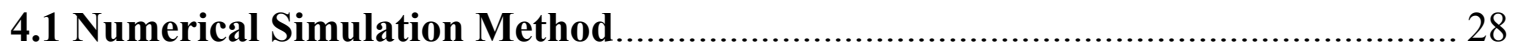

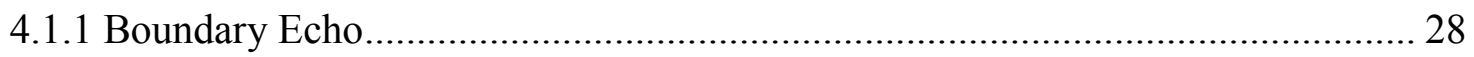

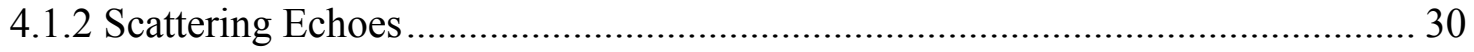

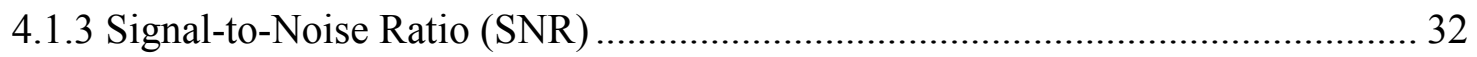

4.1.4 Tissue Boundary Motion............................................................................ 34

4.1.5 Root Mean Squared Error (RMSE) ....................................................... 37

4.1.6 Parameter Setting For Numerical Simulation .............................................. 37

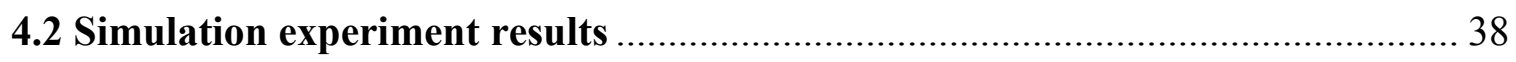

4.2.1 No Boundary Motion with Random Scattering Echoes.................................. 39

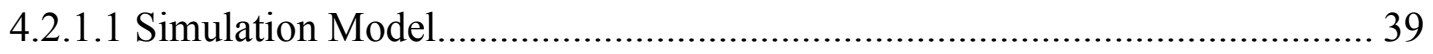

4.2.1.2 Displacement Estimation Results ...................................................... 43

4.2.1.3 Root Mean Squared Error (RMSE) .................................................. 46

4.2.2 Sinusoidal Boundary Motion with Fixed Scattering Echoes ............................ 49

4.2.2.1 Simulation Model............................................................................... 49

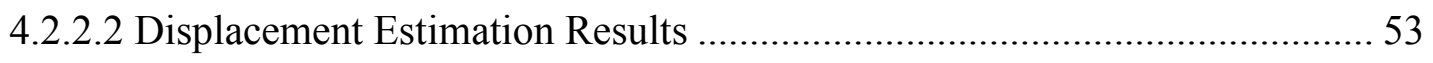


4.2.2.3 Root Mean Squared Error (RMSE)

4.2.3 Sinusoidal Boundary Motion with Random Scattering Echoes......................... 58

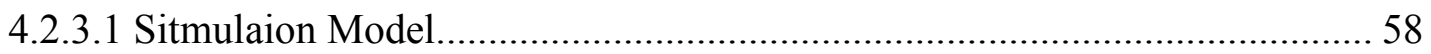

4.2.3.2 Displacement Estimation Results ..................................................... 61

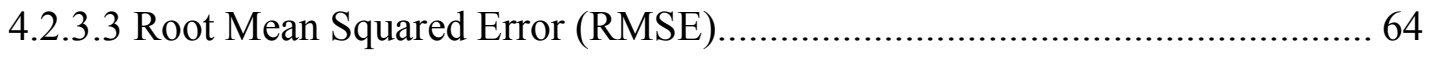

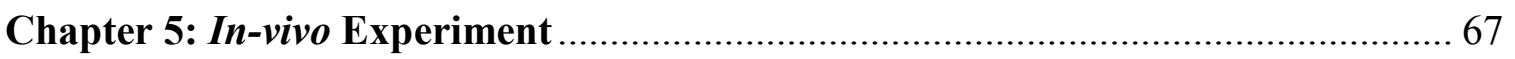

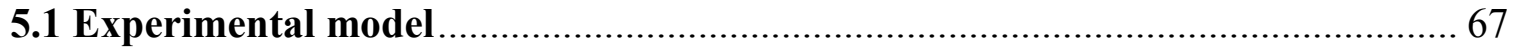

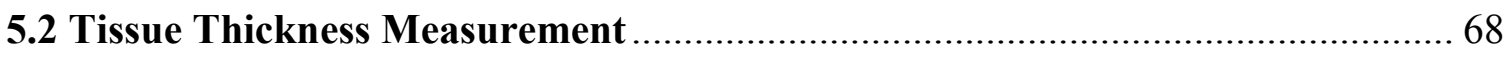

5.3 Forearm Anatomy and US Sensor Placement ................................................ 69

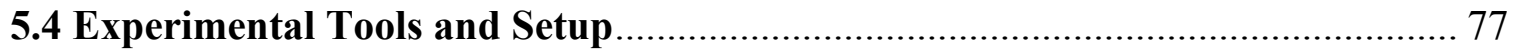

5.4.1 Wearable Ultrasonic Sensor................................................................... 77

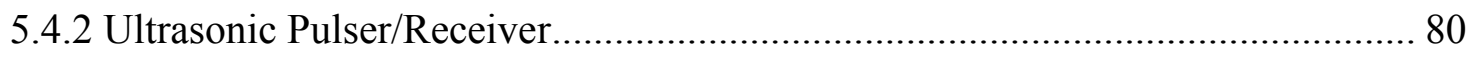

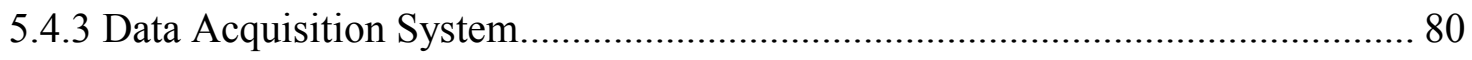

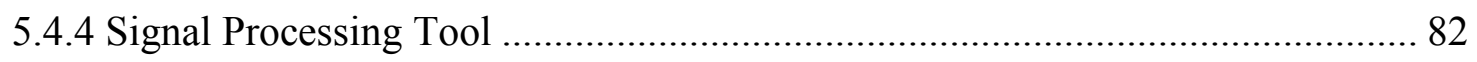

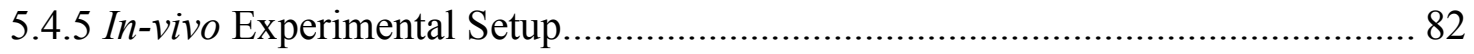

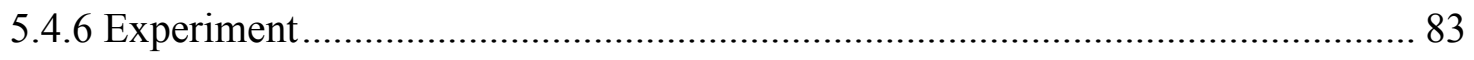

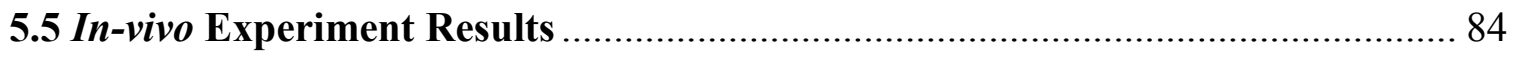

5.5.1 M-mode Ultrasonic Measurement Result .................................................... 84

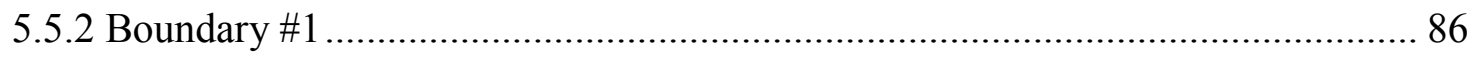


5.5.3 Boundary \#2

5.5.4 Boundary \#3 92

5.5.5 Boundary $\# 4$........................................................................................... 96

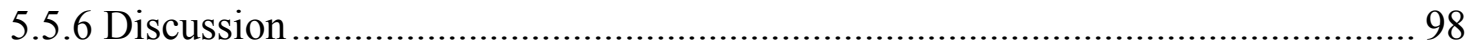

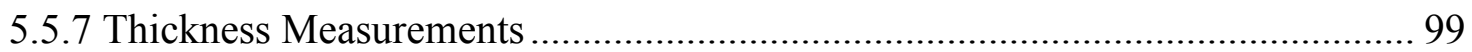

Chapter 6: Conclusions and Future Works ..................................................... 103

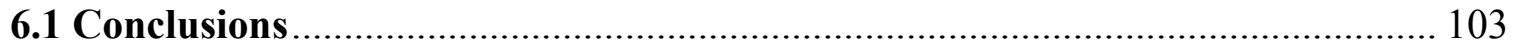

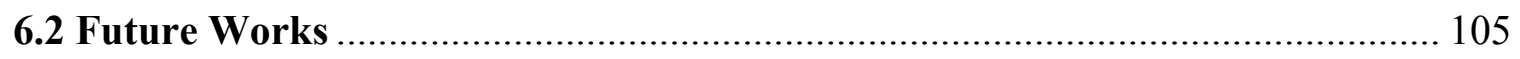

Appendix A: LQ-factorization Technique Frequency Spectrum ......................... 106

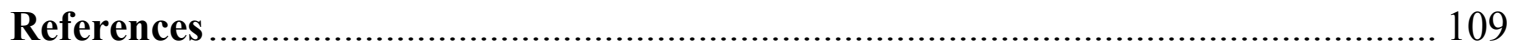




\section{List of Figures and Tables}

Figure 3.1: Ultrasonic pulse-echo measurement model. $T S_{i}$ denotes the $i^{\text {th }}$ tissue layer, and $v_{i}$ is the acoustic velocity in $T S_{i}, T H_{i, j}$ is the $\mathrm{TS}_{\mathrm{i}}$ thickness, and $E_{i, j}$ is the boundary

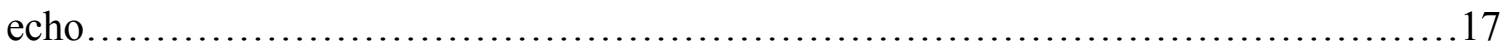

Figure 3.2: Schematic representation of two measurement frames $j$ and $j+1$. a) tissue boundary locations in space domain , b) tissue boundary echoes in time domain. $\mathrm{B}_{\mathrm{i}, \mathrm{j}}$ denotes the tissue boundary, and $E_{i, j}$ denotes the tissue boundary echo. $\mathrm{d}_{\mathrm{i}, \mathrm{j}}$ is the echo boundary $\left(B_{i, j}\right)$ depth, $T_{i, j}$ is the time delay of the boundary echo, $\Delta T d_{i, j}$ is the time delay difference between two consecutive boundary echoes in the same frame, $\Delta T c_{i, j}$ is the time delay change of the boundary echo between two consecutive frames, and $\Delta d_{i, j}$ is the

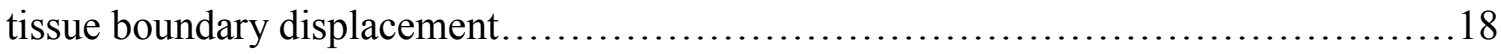

Figure 3.3: Scattering echoes model........................................ 19

Figure 3.4: Time window setup in signal processing to detect a selected boundary echo. $T W l_{i, j}$ is the lower window limit, $T W l_{i, j}$ is the higher window limit, for the boundary

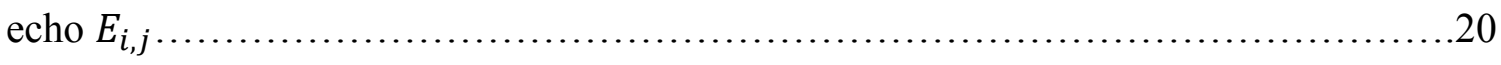

Figure 3.5: Echo peak indicates the tissue layer boundary $\ldots \ldots \ldots \ldots \ldots \ldots \ldots \ldots \ldots \ldots .21$

Figure 4.1: Simulated Gaussian rf pulse echo which represents the tissues boundary echo. .30

Figure 4.2: Simulated scattering echoes.................................... 32 
Figure 4.3: The SNR of the simulated data (Gaussian rf pulse echo + scattering echoes). .34

Figure 4.4: Sinusoidal boundary echo motion. The Gaussian rf pulses are shifted by the value of sinusoidal signal in each point sample... .36

Figure 4.5: The simulation model for the no displace of tissue boundary between two tissue layers $\left(\boldsymbol{T} \boldsymbol{S}_{\mathbf{1}}\right.$ and $\left.\boldsymbol{T} \boldsymbol{S}_{\mathbf{2}}\right)$ with the scattering echoes .39

Figure 4.6: All 100 frames (10 sec at $10 \mathrm{~Hz}$ frame rate) of simulated boundary echoes with no boundary motion having random scattering echoes with $30 \mathrm{~dB}$ SNR in M-mode format. . .40

Figure 4.7: All frames of simulated boundary echoes with no boundary motion having random scattering echoes with $30 \mathrm{~dB}$ SNR in A-mode format.

Figure 4.8: No motion with random scattering echoes simulation M-mode format (a: 25 $\mathrm{dB}, \mathrm{b}: 20 \mathrm{~dB}, \mathrm{c}: 15 \mathrm{~dB}, \mathrm{~d}: 10 \mathrm{~dB}$, e: $5 \mathrm{~dB}$, and f: $0 \mathrm{~dB}$ ).

Figure 4.9: Gaussian rf pulse boundary echo with scattering echoes in $30 \mathrm{~dB}$ SNR (left), and $10 \mathrm{~dB}$ SNR (right)....

Figure 4.10: Boundary track of each technique in seven different SNR values of 30, 25, 20, 15, 10, 5, and $0 \mathrm{~dB}$ (a: echo peak detection, b: cross-correlation between firs frame and all frames, c: cross-correlation between two consecutive frames, d: single frequency phase detection, e: mean of narrow band frequencies phase detection, and f: LQfactorization). .44

Figure 4.11: The comparisons between applying the techniques on raw signals and on signals absolute value for boundary depth estimation for simulation 1 where a) echo peak 
detection, b) cross-correlation between first frame and all frames, and c) cross-correlation between two consecutive frames..................................................

Figure 4.12: RMSE values of all techniques including the absolute value analysis for $\mathrm{A}, \mathrm{S}$ and $\mathrm{F}$ in $30,25,20,15,10,5$, and $0 \mathrm{~dB}$ SNR (A: echo peak detection, S: cross-correlation between first frame and all frames, F: cross-correlation between two consecutive frames, P: single frequency phase detection, MP: mean of narrow band frequencies phase detection, LQ: LQ-factorization, and ABS: absolute value).

Figure 4.13: The same results in Figure 4.11 but without the result of the crosscorrelation between two consecutive frames $(\mathrm{F})$ .48

Figure 4.14: The theoretical value (model) motion track of this simulation 50

Figure 4.15: The simulation model of the Sinusoidal boundary motion with fixed scattering echoes simulation and the Sinusoidal boundary motion with random scattering echoes. Tissue 2 (TS2) is the contracted muscle. (Right figure is confusing. Why there are two boundary echoes? Delete the one at $2.76 \mathrm{~mm}$.) .50

Figure 4.16: : All 100 frames (10 sec at $10 \mathrm{~Hz}$ frame rate) of simulated boundary echoes with sinusoidal boundary motion having fixed scattering echoes with $30 \mathrm{~dB}$ SNR in Mmode format .51

Figure 4.17: All frames of simulated boundary echoes with sinusoidal boundary motion having fixed scattering echoes with $30 \mathrm{~dB}$ SNR in A-mode format.

Figure 4.18: Sinusoidal boundary motion with fixed scattering echoes simulation Mmode format (a: $25 \mathrm{~dB}, \mathrm{~b}: 20 \mathrm{~dB}$, c: $15 \mathrm{~dB}$, d: $10 \mathrm{~dB}$, e: $5 \mathrm{~dB}$, and f: $0 \mathrm{~dB}$ ) .53 
Figure 4.19: Boundary track of each technique in seven different SNR values of 30, 25, 20, 15, 10, 5, and $0 \mathrm{~dB}$ (a: echo peak detection, b: cross-correlation between firs frame and all frames, c: cross-correlation between two consecutive frames, d: single frequency phase detection, e: mean of narrow band frequencies phase detection, and f: LQ-

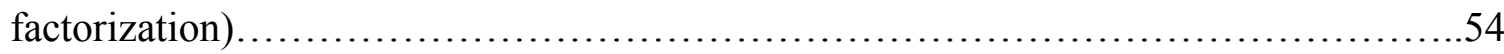

Figure 4.20: The comparisons between applying the techniques on raw signals and on signals absolute value for boundary depth estimation for simulation 2 where a) echo peak detection, b) cross-correlation between first frame and all frames, and c) cross-correlation

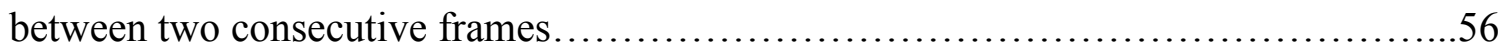

Figure 4.21: RMSE values of all techniques including the absolute value analysis for $\mathrm{A}, \mathrm{S}$ and $\mathrm{F}$ in $30,25,20,15,10,5$, and $0 \mathrm{~dB}$ SNR (A: echo peak detection, S: cross-correlation between first frame and all frames, F: cross-correlation between two consecutive frames, P: single frequency phase detection, MP: mean of narrow band frequencies phase detection, LQ: LQ-factorization, and ABS: absolute value)....

Figure 4.22: RMSE values of only single frequency phase detection $(\mathrm{P})$, mean of narrow

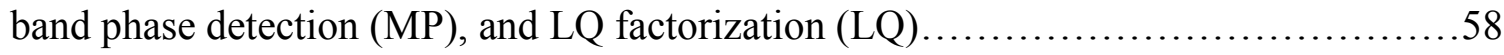

Figure 4.23: All 100 frames (10 sec at $10 \mathrm{~Hz}$ frame rate) of simulated boundary echoes with sinusoidal boundary motion having random scattering echoes with $30 \mathrm{~dB}$ SNR in Mmode format .59

Figure 4.24: All frames of simulated boundary echoes with sinusoidal boundary motion having random scattering echoes with $30 \mathrm{~dB}$ SNR in A-mode format....

Figure 4.25: Sinusoidal boundary motion with random scattering echoes simulation $\mathrm{M}$ mode format (a: $25 \mathrm{~dB}, \mathrm{~b}: 20 \mathrm{~dB}, \mathrm{c}: 15 \mathrm{~dB}, \mathrm{~d}: 10 \mathrm{~dB}$, e: $5 \mathrm{~dB}$, and f: $0 \mathrm{~dB}$ ) 
Figure 4.26: track of each technique in seven different SNR values of 30, 25, 20, 15, 10, 5, and $0 \mathrm{~dB}$ (a: echo peak detection, b: cross-correlation between firs frame and all frames, c: cross-correlation between two consecutive frames, d: single frequency phase detection, e: mean of narrow band frequencies phase detection, and f: LQfactorization) .62

Figure 4.27: The comparisons between applying the techniques on raw signals and on signals absolute value for boundary depth estimation for simulation 3 where a) echo peak detection, b) cross-correlation between first frame and all frames, and c) cross-correlation between two consecutive frames .64

Figure 4.28: RMSE values of all techniques and the absolute value analysis in 30, 25, 20, 15, 10, 5, and $0 \mathrm{~dB}$ SNR (A: echo peak detection, S: cross-correlation between first frame and all frames, F: cross-correlation between two consecutive frames, P: single frequency phase detection, MP: mean of narrow band frequencies phase detection, LQ: LQfactorization, and ABS: absolute value).

Figure 4.29: RMSE values of only single frequency phase detection $(\mathrm{P})$, mean of narrow band phase detection (MP), and LQ factorization (LQ) .66

Figure 5.1: Ultrasonic pulse-echo measurement model of in-vivo experiment for tracking tissue boundaries.

Figure 5.2: Supination and pronation position of right forearm [107] ................ 71

Figure 5.3: Muscle structures of the right forearm in posterior view $[107] \ldots \ldots \ldots \ldots . . .72$

Table 5.1: Forearm's component of tissues in anterior and posterior sides and tissue level [106-109].... 
Figure 5.4: a) 1- lateral epicondyle, 2- medial epicondyle, 3- supinator muscle, 4pronator teres muscle, 5- radius, 6- ulna, and 7- pronator quadrates muscle. b) 1- superior and inferior ulnar collateral branches of brachial artey, 2- middle collateral branch of deep brachial artery, 3- medial intermuscular septum, 4- lateral intermuscular septum, 5ulnar nerve, 6- brachioradialis muscle, 7- posterior ulnar recurrent artery, 8- lateral epicondyle of humerus, 9- medial epicondyle of humerus, 10- extensor carpi radialis longus muscle, 11- triceps brachii tendon (cut), 12- common extensor tendon (partially cut), 13- olecranon of ulna, 14- extensor carpi radialis brevis muscle, 15- anconeus muscle, 16- supinator muscle, 17- interosseous recurrent artery, 18- deep branch of radia nerve, 19- flexor carpi ulnaris muscle, 20- pronator teres muscle (slip of insertion), 21posterior interosseous artery, 22- radius, 23- ulna, 24- posterior interosseous nerve, 25extensor pollicis longus muscle, 26- abductor pollicis longus muscle, 27- extensor indicis muscle, 28- extensor pollicis brevis muscle, 29- anterior interosseous artery (termination), 30- extensor carpi radialis brevis tendon, 31- extensor carpi ulnaris tendon (cut), 32extensor carpi radialis longus tendon, 33- extensor digiti minimi tendon (cut), 34- radial artery, 35- extensor digitorum tendons (cut), 36- $1^{\text {st }}$ metacarpal bone, 37- extensor retinaculum, 38- $2^{\text {nd }}$ metacarpal bone, 39- $5^{\text {th }}$ metacarpal bone, and $40-1^{\text {st }}$ dorsal interosseous muscle. c) 1- triceps brachii muscle, 2- olecranon of ulna, 3-posterior ulnar recurrent artery, 4- brachioradialis muscle, 5- medial epicondyle of humerus, 6- extensor carpi radialis longus muscle, 7- ulnar nerve, 8- common extensor tendon, 9- anconeus muscle, 10- extensor carpi radialis brevis muscle, 11- flexor carpi ulnaris muscle, 12extensor digitorum muscle, 13- extensor carpi ulnaris muscle, 14- abductor pollicis longus muscle, 15- extensor digiti minimi muscle, 16- extensor pollicis brevis muscle, 17- extensor retinaculum, 18- extensor pollicis longus tendon, 19- dorsal branch of ulnar nerve, 20- extensor carpi radialis brevis tendon, 21- extensor carpi ulnaris tendon, 22extensor carpi radialis longus tendon, 23- extensor digiti minimi tendon, 24- superficial branch of radial nerve, 25- extensor digitorum tendons, 26- abductor pollicis longus tendon, 27- $5^{\text {th }}$ metacarpal bone, 28- extensor pollicis brevis tendon, 29- extensor indicis tendon, 30- anatomical snuffbox, and 31- extensor pollicis longus tendon [107].........76 
Figure 5.5: Cross-sectional MRI image anatomy of the proximal forearm: 1) radius, 2) ulna, 3) flexor digitorum profundus, 4) ulnar nerve, 5) flexor carpi ulnaris, 6) flexor digitorum superficialis, 7) Palmaris longus, 8) flexor carpi radialis, 9) pronator teres, 10) median nerve, 11) supinator, 12) radial nerve, superficial branch, 13) brachioradialis, 14) extensor carpi radialis longus, 15) extensor carpi radialis brevis, 16) radial nerve, deep branch, 17) extensor digitorum, 18) extensor carpi ulnaris, and 19) anconeus [106]......77

Figure 5.6: Schematic representation of the wearable ultrasonic sensor structure [111]..79

Figure 5.7: Photographs of the wearable ultrasonic sensor. A) top view, and b) side view while bending [111] 79

Figure 5.8: The used ultrasonic pulser/receiver device (NDT 5900PR)...............80

Figure 5.9: a) PCI Digitizer and b) acquisition program........................... 81

Figure 5.10: Wearable ultrasonic sensor attached on the right proximal forearm a) muscle

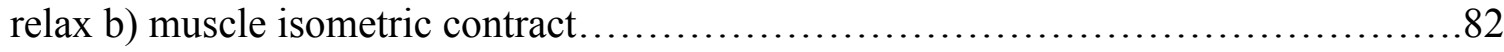

Figure 5.11: The experiment setup in the ultrasonic pulse-echo mode.................83

Figure 5.12: The experimental M-mode ultrasonic measurement image, where $T S_{i}$ is the

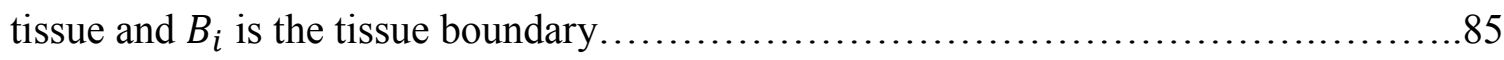

Figure 5.13: All frames raw signal showing the location of the tissue boundary echoes................................................................... 86

Figure 5.14: Boundary echo from $B_{1}$ in M-mode image.......................... 87 
Figure 5.15: Frequency spectrum of $E_{1}$, where $f l$ is the low frequency of the narrow band, $f h$ is the high frequency, and $f c$ is the center frequency........................... 88

Figure 5.16: Depth of tissue boundary $B_{1}$ obtained by: a) echo peak detection, b) crosscorrelation between first frame and all frames, c) cross-correlation between two consecutive frames, d) phase detection using single frequency, e) phase detection using the mean of narrow band frequencies, and f) LQ-factorization.

Figure 5.17: Boundary echo from $B_{2}$ in M-mode image.

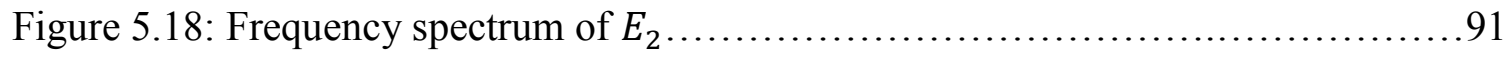

Figure 5.19: Depth of tissue boundary $B_{2}$ obtained by: a) echo peak detection, b) crosscorrelation between first frame and all frames, c) cross-correlation between two consecutive frame, d) single frequency phase detection, e) mean of narrow band frequencies phase detection, and LQ-factorization techniques... 92

Figure 5.20: Boundary echo from $B_{3}$ in M-mode image .93

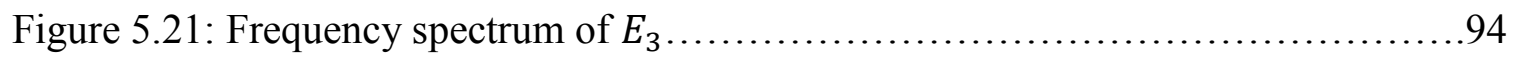

Figure 5.22: Depth of tissue boundary $B_{3}$ obtained by: a) echo peak detection, b) crosscorrelation between first frame and all frames, c) cross-correlation between two consecutive frame, d) single frequency phase detection, e) mean of narrow band frequencies phase detection, and LQ-factorization techniques......................95

Figure 5.23: Boundary echo from $B_{4}$ in M-mode image...........................96

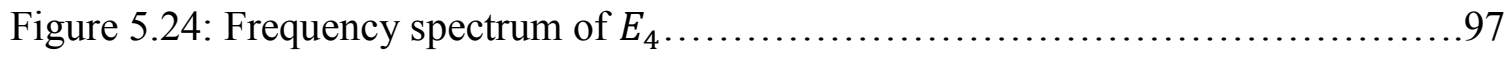


Figure 5.25: Depth of tissue boundary $B_{4}$ obtained by: a) echo peak detection, b) crosscorrelation between first frame and all frames, c) cross-correlation between two consecutive frame, d) single frequency phase detection, e) mean of narrow band frequencies phase detection, and f) LQ-factorization techniques.....................98

Figure 5.26: Tissue boundary depths obtained by the LQ-factorization technique........100

Figure 5.27: Thickness variations of each tissue layer due to muscle contraction.......101

Figure 5.28: Total thickness changes between the ultrasonic sensor and the bone........102

Figure A.1: The frequency spectrum of the $E_{1}$ a) the acquired signals, b) the separated signals at the center frequency, and c) the acquired signals without the center frequency

Figure A.2: The frequency spectrum of the $E_{2}$ a) the acquired signals, b) the separated signals at the center frequency, and c) the acquired signals without the center frequency.

Figure A.3: The frequency spectrum of the $E_{3}$ a) the acquired signals, b) the separated signals at the center frequency, and c) the acquired signals without the center

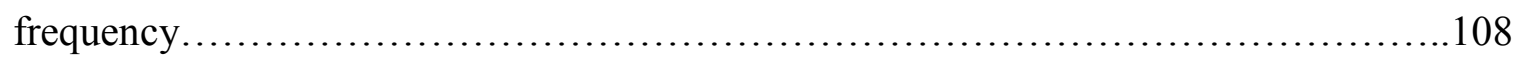

Figure A.4: The frequency spectrum of the $E_{4}$ a) the acquired signals, b) the separated signals at the center frequency, and c) the acquired signals without the center frequency............................................................. 108 


\section{List of Abbreviations}

\begin{tabular}{|c|c|}
\hline Abbreviations & Definitions \\
\hline MMG & Mechanomyography \\
\hline EMG & Electromyography \\
\hline sEMG & Surface Electromyography \\
\hline MRI & Magnetic Resonance Imaging \\
\hline $\mathrm{CT}$ & Computed Tomography \\
\hline US & Ultrasound \\
\hline EEG & Electroencephalogram \\
\hline ATP & Adenosine Triphosphate \\
\hline A-mode & Amplitude Mode \\
\hline B-mode & Brightness Mode \\
\hline M-mode & Motion Mode \\
\hline LQ-factorization & Lower Triangular and Orthogonal Factorization \\
\hline $\mathrm{rf}$ & Radio Frequency \\
\hline SNR & Signal-to-Noise Ratio \\
\hline RMSE & Root Mean Squared Error \\
\hline a.u. & Arbitrary Unit \\
\hline
\end{tabular}

xviii 
ABS

3D
Absolute Value

Three Dimensional 


\section{List of Symbols}

$d_{i, j} \quad$ Tissue boundary depth of $i^{\text {th }}$ echo boundary in $j^{t h}$ frame

$v_{i} \quad$ Acoustic velocity in the $i^{\text {th }}$ tissue

$T_{i, j} \quad$ Time delay of the echo boundary

$T H_{i, j} \quad$ Tissue thickness

$\Delta d_{i, j} \quad$ Displacement of the $i^{\text {th }}$ echo boundary between the frame numbers $j+1$ and $j$

$\Delta T c_{i, j} \quad$ Time delay change of $i^{t h}$ echo boundary

$\Delta T d_{i, j} \quad$ Time delay difference between the frame numbers $j+1$ and $j$

$i \quad$ Tissue or boundary number

M Total number of tissues or boundaries

j Frame number corresponding to measurement time

$F \quad$ Total number of frames relative to measurement time

$T S_{i} \quad$ Tissue number

$E_{i, j} \quad$ Tissue boundary echo

$B_{i, j} \quad$ Tissue boundary

$A_{i, j} \quad$ Amplitude of the boundary echo 
$S_{i, j} \quad$ Measured signals

$T W l_{i, j} \quad$ Low window limit

$T W h_{i, j} \quad$ High window limit

C Correlation between two frames

$G_{i, j} \quad$ Delay between two frames at a correlation $C$

$\varphi_{i, j} \quad$ Unwrap phase

$f c_{i} \quad$ Chosen frequency (center frequency)

$\varphi r_{i, j} \quad$ Unwrap phase with the first frame phase referenced

$\lambda_{i, j} \quad$ The chosen frequency wavelength

$d r_{i, j} \quad$ Boundary depth where the depth of the first frame is zero

$f l_{i, j} \quad$ The low limit of the frequency bandwidth

$f h_{i, j} \quad$ The high limit of the frequency bandwidth

$\varphi_{a v e} e_{i, j} \quad$ Average phases of multiple frequencies (within the bandwidth)

$f q_{i} \quad$ Selected frequency for the LQ-factorization technique

$q \quad$ The selected frequency number

a The total number of selected frequencies for the LQ-factorization technique

$S f q_{i, j} \quad$ Generated signal of the selected frequency $f q_{i}$ 
A Signal amplitude value of $S f q_{i, j}$

$\vartheta \quad$ The initial phase value of $S f q_{i, j}$

$T_{S} \quad$ Ultrasonic rf signal sample period

$L \quad$ Lower triangular matrix

$Q \quad$ Orthogonal unity matrix

$b_{q} \quad$ Elements of the $L$ matrix

$d S_{i, j} \quad$ The separated signal at the selected frequency or frequencies

$\grave{L} \quad$ Matrix taken from $L$

Q $\quad$ Matrix taken from $Q$

$R \quad$ The total number of frames $(F)$ plus the total number of selected

frequencies for the LQ-factorization technique (a)

$\beta \quad$ Bandwidth factor

$\omega c_{i} \quad$ Center angular frequency of the boundary echo

$t \quad$ Time after pulse transmission

TT Total acquisition time for each frame corresponding to the tissue depth

$n \quad$ Ultrasound rf signal sample index

$N \quad$ The total sample number corresponding to the tissue depth

$s E_{m, j} \quad$ Signal scattering echo from $m^{\text {th }}$ scatterer 
$\alpha_{m, j} \quad$ Amplitude factor

$m \quad$ The index number of scatterer in simulations

$J \quad$ Total number of scatterers within the ultrasonic beam

$\tau_{m, j} \quad$ Time delay between the ultrasonic sensor and $m^{\text {th }}$ scatterer

$A_{m, j} \quad$ Amplitude of the signal scattering echo from $m^{t h}$ scatterer

$\theta_{m, j} \quad$ Phase of $m^{\text {th }}$ scattering echo

$D_{m, j} \quad$ The distance between the ultrasonic sensor and the $m^{\text {th }}$ scatterer

$s E r_{m, j} \quad$ Received scattering echoes from scatterer $m$

$s S_{i, j} \quad$ The simulated signals (tissue boundary echo + received scattering echoes)

SM Sinusoidal displacement of the tissue boundary

$S M_{0} \quad$ The initial boundary depth of the simulated sinusoidal boundary motion

$A_{N} \quad$ Magnitude of displacement

$f m \quad$ The frequency of $S M$ signal

SNR Signal-to-Noise Ratio

$\hat{A}_{b} \quad$ Maximum amplitude of the absolute value of the boundary echo

$\bar{A}_{s} \quad$ Average magnitude of the scattering echoes

RMSE Root Mean Squared Error 
$M T_{j} \quad$ Boundary displacement value obtained from the techniques

$\widehat{M T}_{j} \quad$ Theoretical value (model) of the boundary displacement

$T H_{\text {total }_{, j}}$ Total thickness between ultrasonic sensor and bone 


\section{Chapter 1}

\section{Introduction}

This chapter presents an overview of this thesis and includes the research objectives, contributions and thesis organization.

\subsection{Overview}

The wide usage of ultrasonic imaging in creating internal human body images noninvasively with its low cost has made it an essential technique in many research fields. Ultrasonic imaging has been used in many studies to monitor muscle motion and to measure its thickness variation. Measuring the displacement of the tissue boundaries can provide useful information about skeletal muscle performance which helps in many medical and clinical applications such as the diagnosis of neuromuscular disease, rehabilitation, therapy, and prosthesis control.

In order for one to perform an action or movement, muscle contraction is required, and this results in changes of muscle thickness. Moreover, muscle thickness variation has been observed in many medical cases such as neuromuscular disorders $[1,2]$ and injury [3], assessment of muscle fatigue [4] and monitoring of muscle motion $[5,6]$, which shows the importance and need to do research more deeply in the mechanism of muscle thickness variation. There are many modalities that can measure change in muscle 
thickness such as MRI, CT, and X-ray. However, these modalities have either risk of radiation exposure or high costs to operate. The aim of this research is to measure tissue boundary displacement and tissue layer thickness variation using a wearable ultrasonic sensor developed in our research group [7].

\subsection{Problem Statement}

In this research, the wearable ultrasonic sensor is used in the in-vivo experiment. The ultrasonic sensor was constructed using a piezoelectric polymer in order to achieve its flexibility and lightweight. While, a conventional ultrasonic probe is typical constructed using a piezoelectric ceramic, therefore it is bulky, rigid, and heavy. However, the energy of the transmitted ultrasonic waves using the polymer sensor is lower than the ceramics sensor. The low energy of the ultrasonic waves results in low amplitude echoes, therefore, the signal to noise ratio (SNR) of the desired signals (tissue boundary echoes) become lower. Low SNR makes tracking tissue boundary echoes more challenging [8].

\subsection{Objectives}

The objectives of this thesis are to improve the measurement accuracy of internal tissue boundary displacement that is obtained by a wearable ultrasonic sensor made from piezoelectric polymer material using the signal processing approach and to continuously monitor the thickness changes of internal tissue layers during muscle contraction quantitatively. 
Signal analysis techniques to measure tissue boundary displacement are tested and compared in order to find most reliable and accurate technique that can overcome the challenges and limitations of the wearable ultrasonic sensor. The techniques investigated are echo peak detection, cross-correlation, phase detection, and LQ-factorization. These techniques were chosen because they were used in other studies of monitoring muscle thickness, while the LQ factorization is a new technique applied for muscle thickness monitoring for the first time.

\subsection{Thesis Contributions}

The main contributions of this thesis are presented as follows:

- Overcome the limitations of the wearable ultrasonic sensor using the signal processing technique approach.

- Investigating the effect of scattering echoes on the motion tracking accuracy by numerical simulation and in-vivo experiments.

- A comparison of chosen signal processing techniques in obtaining tissue boundary displacement quantitatively.

\subsection{Research Publications}

- Hisham Turkistani, Ibrahim AlMohameed, and Yuu Ono "Continuous Monitoring of Muscle Thickness Changes during Isometric Contraction Using a Wearable 
Ultrasonic Sensor," Conference of the Canadian Medical and Biological

Engineering Society, Ottawa, Canada, May 21-24, 2013.

\subsection{Thesis Organization}

Chapter 1: The introduction of the thesis including the objectives, problem statement, and the contribution.

Chapter 2: Background information about human muscle and their types of contractions, muscle monitoring measurement methods, and signal processing techniques.

Chapter 3: Description of the ultrasound measurement method and the signal processing techniques applied for measuring tissues boundary displacements and tissues thickness.

Chapter 4: Investigation of the effects of the scattering echoes on the accuracy of the tissues boundary echo tracking by means of numerical simulation experiments.

Chapter 5: In-vivo experiment of boundary echo tracking and tissue layer thickness measurements at the forearm of a human subject.

Chapter 6: A summary of this thesis study of the tissues boundary motion tracking and tissue layer thickness measurement during muscle contraction. The future work is also presented. 


\section{Chapter 2}

\section{Technical Background Review}

In this chapter, background information of human muscles is presented. Afterwards, several methods for muscle monitoring are introduced and compared. Finally, selected signal processing techniques applied in this thesis research are presented.

\subsection{Muscle Contraction}

There are three types of muscles: skeletal muscles, smooth muscles, and cardiac muscles. A skeletal muscle is responsible for body movements. Skeletal muscles are connected to bones by tendons, so they support and move the skeleton. Skeletal muscles are usually voluntary control muscles and are contracted by generating electrical impulses in the neurons. Smooth muscles are involuntary control muscles. Autonomic nervous system controls the smooth muscle. Smooth muscles are responsible for the contractility of hollow organs, such as blood vessels, the gastrointestinal tract, the bladder, or the uterus. The contraction speed is slow compared to the speed of skeletal muscle contractions. Cardiac muscles are the heart muscles and they are involuntary muscles. Cardiac muscles contract continuously and in a rhythm for the entire life of an organism [9]. 
When skeletal muscles are contracted, their forces, shapes, lengths, stiffness and/or thickness may be changed. These changes vary by types of contractions in each muscle. There are two main types of muscle contractions: static and dynamic [9-11]. In static contraction, there is no body movement involved, but muscle force and thickness still change. This is because the muscle tension or force exerted is equal to an external load (e.g. pushing against a wall). Static contraction is also called isometric contraction (iso: same, metric: length) since the length of the muscle does not change during contraction. In dynamic contraction, there are four types: auxotonic, isotonic, isokinetic and plyocentric. Auxotonic contraction (auxo: increased, tonos: tension) is a dynamic contraction where muscle tension changes constantly because of the continual change of joint angle and speed of the movement. An example of auxotonic contraction is lifting a barbell. Isotonic contraction (iso: same, tonos: tension) is a rare case of dynamic contraction. In the isotonic contraction, the length of the muscle changes but not the tension. This occurs, for example, when an athlete lowers a heavy barbell slowly with a constant speed. Muscles also can be contracted with a constant motion in the isokinetic contraction (iso: same, kinetic: motion). This occurs when someone lifts a heavy object with a constant speed. In this case, muscles release tension with different joint angle to maintain the same motion. Finally, the plyocentric contraction is actually an isotonic contraction but from a muscle stretched position. This contraction maintains the muscle not to stretch too much. Athletes use this type of contraction for training, such as jumping to increase muscle's potential and loses a great amount of calories. 


\subsection{Methods of Muscle Monitoring}

Muscle monitoring has been an interest for many researchers and physicians. It provides a wide range of information about muscles. Therefore, it is used in various medical and clinical applications, such as diagnoses of neuromuscular disorders, rehabilitation, physical therapy and prosthetic control. There are several common measurement methods that are used to monitor the muscle: mechanomyography (MMG), electromyography (EMG), magnetic resonance imaging (MRI), X-ray, Computed Tomography (CT), and ultrasound (US).

\subsubsection{Mechanomyography (MMG)}

MMG detects the mechanical surface motion of the skeletal muscle that results from skeletal muscle contraction $[12,13]$. It uses different types of sensors to record the mechanomyographic signal such as microphones, piezoelectric contact sensors, accelerometers and laser distance sensors [13, 14].

\subsubsection{Electromyography (EMG)}

EMG is a measurement of a muscle's electrical activities during skeletal muscle contractions [15]. EMG comes with two different measurement types: invasive and noninvasive [16, 17]. Invasive EMG uses a needle electrode to detect the electrical activity of 
a local region of a selected muscle. Non-invasive EMG, also called surface EMG (sEMG), measures the summation of electrical activities or signal arising from active muscles under non-invasive electrodes directly placed on the skin. The sEMG technique is demonstrated to determine the muscle force, length [18, 19] and action potential conduction velocity [20-22]. The invasive EMG has better spatial resolution than sEMG since it detects the electric activity of small and local region of muscles. However, sEMG is widely used due to its non-invasiveness.

\subsubsection{Magnetic Resonance Imaging (MRI)}

MRI can take an image of the internal body non-invasively and painless with high spatial resolution. MRI provides the internal muscle structure. This technology uses a large magnet, radio waves and a computer to scan the body. [23-26]. However, MRI is high cost, and does not capture real time images.

\subsubsection{X-ray}

$\mathrm{X}$-ray is able to visualize body structures because the X-ray attenuation depends on the type of body tissues. X-ray has been used to measure the X-ray diffraction differences between resting and rigor muscles by measuring the X-ray diffraction using low-angle scattering system [27-31]. It is also used in obtaining the total-body skeletal 
muscle mass for studying the nutritional, physiologic, and metabolic of the skeletal muscle [32-34].

\subsubsection{Computed Tomography (CT)}

$\mathrm{CT}$ is a scanning technique that uses X-rays to take highly detailed images of the body. CT imaging has been widely used in identifying body structures and in neuromuscular disorder diseases. It has also been used to measure muscle thickness changes and lunge volume changes [35-37]. CT scans provide 3D images and real time images [37]. CT scans can work in conjunction with positron emission tomography (PET) scans in cardiovascular diseases and for clinical oncology [38-40].

\subsubsection{Ultrasound (US)}

Ultrasound is a non-invasive, less harmful and relatively low-cost technique. It can observe the structure and motion of muscles and other tissues in real-time with a high frame rate. Ultrasonic methods have been applied for diagnosis of neuromuscular disorders [1,2] and injury [3], assessment of muscle fatigue [4] and monitoring of muscle motion $[5,6]$. It can identify tendons, muscles, joints, vessels and internal organs for possible pathology or lesions. It is also being used for the measurement of tissue stiffness, as in elastography imaging [10]. 


\subsubsection{Current Research and Applications}

In recent studies, one or a combination of the above methods have been applied in monitoring muscle activities [41]. The selection of the method depends on the main focus and what kind of information is needed. EMG has been used in gait analysis to measure abnormalities in movement due to diseases $[42,43]$ Muscle fatigue can be evaluated by EMG [44, 45] and MMG [46]. EMG is also used with MMG to measure both electrical and mechanical activities during muscle contraction for resistance-trained and aerobically-trained subjects during a fatiguing muscle action [41, 47-49]. MRI is less used compared to other mentioned methods due to its high operating cost and limitations with real time measurements. MRI is used to image interior muscle structures in detail $[50,51]$. X-ray has been used to in a research to find out how the molecular motor in muscle converts the free energy of ATP hydrolysis into work during muscle contraction [52]. CT scanner used to monitor muscle growth and tissue changes quantitatively. Also it was used to monitoring of muscle and bone recovery in spinal injury patients $[53,54]$.

Because of its low cost, non-invasiveness and less harmful operation, ultrasound imaging is widely used in many medical and clinical applications such as measurement of muscle tendon length $[55,56]$, blood flow [57-59], motion tracking $[60,61]$ and threedimensional imaging of muscle structure [62]. In addition, it has been used along with vibration probes to determine muscle and tissue stiffness in liver fibrosis and cancers [10]. It has been successfully used in measuring muscle fatigue as well $[63,64]$. Ultrasound imaging has also been used in conjunction with EMG to determine the time delay 
between the electrical and mechanical activities of the muscle to determine the onset of muscle fascicles and tendon motion induced by electrical stimulation [65].

\subsection{Advantages of US Method}

US imaging in muscle studies is mostly compared with MRI $[37,67,68]$ and EMG $[69,70]$. There is a positive correlation between the results obtained by MRI and US imaging $[69,70]$. US image also has strong correlation with CT image. US is safer than the CT because of no radiation exposures. $[67,71]$. Most of the relevant studies describe US imaging as an accurate, reliable and valid measurement tool in muscle thickness monitoring [37, 41, 67-71]. US imaging data demonstrated a strong correlation with muscle contraction and can provide useful information of muscle contraction and may complement with EMG and MMG techniques [41]. MMG has poor spatial resolution and can only provide surface motion data. sEMG can measure only the average electric muscle activities. X-ray and CT scans have high risk of radiation exposures. On the other hand, US imaging is inexpensive, less harmful, portable, and is a comfortable modality along with its feasibility and accuracy. Therefore, US imaging is a promising candidate for muscle thickness monitoring.

\subsection{US for Muscle Displacement and Thickness Measurement}

The study of muscle monitoring has become an interest of many researches and is a growing field. The US method is widely applied for muscle thickness measurement [37, 
41, 67-82]. Different research groups have used different terminologies in their studies such as muscle thickness, muscle size, deformation, displacement, muscle cross-sectional and aspect ratio. The terms used in this thesis are muscle thickness and displacement [37, 41, 67-82]. Muscle thickness was obtained through several ultrasonic measurement modes (e.g. M-Mode, A-Mode, B-Mode, and Doppler) [41, 61, 62, 67, 69, 72-77]. In abdominal researches, researchers succeeded to measure the thickness change between rest and contraction $[69,72]$. Muscle thickness change was successfully observed in the investigations of muscle thickness with age and gender using US imaging [78, 79]. Muscle thickness change was also observed in many muscle diseases such as low back pain $[69,80]$, shoulder pain $[68,81]$ and myotonic dystrophy type 2 [82]. Thickness change has also been observed in chest muscles to report the intraexaminer reliability to make thickness measurements of the pectoralis major muscle (serratus anterior and pectoralis major muscles) $[75,76]$. Moreover, US has effectively obtained muscle thickness change of many muscles such as trapezius, pectoralis, serratus, oblique, shoulder, quadriceps, tibial, multifidus, cuff, rectus femoris and forearm muscle [37, 41, 67-82]. In addition, it has been used in the diagnosis and treatment of various urological disorders [67]. As MMG and EMG, ultrasound imaging has been used to assess the behaviour of muscles in muscle fatigue [64]. US imaging was used to evaluate the effect of long-term physical training [71]. Another study investigated US imaging for measuring muscle thickness of pigs to find the US imaging accuracy and feasibility in measuring muscle thickness change to index muscle weakening and atrophy [37]. 


\subsection{Signal Processing Techniques}

Various signal processing techniques to analyse ultrasonic signals have been applied for estimating muscle thickness changes such as cross-correlation and peak detection. In this research, four techniques were tested to track the tissue boundary motion and to measure the tissue thickness changes. The selected techniques are echo peak detection, cross-correlation, phase detection, and LQ-factorization.

\subsubsection{Echo Peak Detection}

Detecting the maximum value in a signal is widely used in many studies. Several methods have been applied to detect the maximum value such as peak detection $[83,84]$, peak recognition [85], maximum likelihood [86, 87], maximum amplitude detection [88], and local and global maximum [89].

\subsubsection{Cross-correlation Techniques}

Cross-correlation is a method to find the best correlation between two signals and to measure the time lag between them. It has been used as a tool to detect tissue displacement in clinical ultrasonic researches $[61,90]$. 


\subsubsection{Phase Detection Techniques}

A number of approaches have been proposed to obtain signal phase in different applications [91-93]. It can provide information about tissues boundaries displacement. This technique is based on Fourier Transform. The signal phase shift in frequency domain can be translated to time delay which corresponds to the displacement of a tissue boundary in this study.

\subsubsection{LQ-factorization}

LQ-factorization (L: Lower triangular, Q: orthogonal matrix) is a computational mathematics to solve linear algebra problems [94-96]. It has been used recently in signal processing. LQ-factorization can decompose a signal into its components, such as signals and noises. One clinical study has successfully improved their system to record auditory steady-state response in electroencephalogram (EEG) in shorter recording duration using the LQ-factorization technique [94, 95]. 


\section{Chapter 3}

\section{Measurement Method of Tissue Displacement and Thickness}

\subsection{Method}

The objective of this thesis research is to track the tissue boundary displacement and tissue layer thickness change using a wearable ultrasonic sensor. As mentioned in Chapter 2, muscle thickness changes during muscle contraction. Tissue boundary depths inside a body can be obtained noninvasively using ultrasound. An ultrasonic pulser/receiver generates electric pulses that are converted into ultrasonic waves by the wearable ultrasonic sensor. The ultrasonic waves propagate through body tissues with a certain velocity and are reflected at tissue boundaries where they are received by the same wearable ultrasonic sensor. The reflection of the ultrasonic waves is caused by the difference of the acoustic impedance of two different tissues [97]. Boundaries beyond the bone cannot be visible easily because of the high reflection coefficient of the bone and the high attenuation of the waves inside the bone. The ultrasonic pulse-echo measurement model is shown in Figure 3.1. TS $i$ denotes the $i^{\text {th }}$ tissue, and $v_{i}$ is the acoustic velocity in $T S_{i}, T H_{i, j}$ is the $T S_{i}$ tissue thickness in the $j^{\text {th }}$ measurement frame, and $E_{i, j}$ denotes the boundary echo. 
Figure 3.2 shows the schematic representation of two consecutive measurement frames $(j$ and $j+1)$ in space and time domains, to define the tissue boundary depth, time delay difference of two echoes in the same frame, time delay change of an echo between two frames, and tissue boundary displacement in our ultrasonic measurements. Each tissue boundary depth and thickness can be obtained by measuring the time delay of the reflected ultrasonic waves (echoes) using the known tissue velocities by:

$$
\begin{gathered}
d_{i, j}=\sum_{k=1}^{i} \frac{v_{k} \cdot \Delta T d_{k, j}}{2} \\
\Delta T d_{i, j}=T_{i, j}-T_{i-1, j}, \text { where } T_{0, j}=0 \\
T H_{i, j}=d_{i, j}-d_{i-1, j}, \text { where } d_{0, j}=0
\end{gathered}
$$

where $d_{i, j}$ is the tissue boundary depth for the $i^{\text {th }}$ boundary echo $\left(E_{i, j}\right)$ in the $j^{\text {th }}$ measurement frame, $\Delta T d_{i, j}$ is the time delay difference between the consecutive boundary echoes of $E_{i-1, j}$ and $E_{i, j}$ in the $j^{\text {th }}$ frame, and $T_{i, j}$ is the time delay of $i^{\text {th }}$ boundary echo.

In addition, a boundary displacement is defined as the depth change of a tissue layer boundary during muscle contraction. The time delay change of each tissue boundary echo and the displacement of each tissue boundary between two consecutive frames can are defined by Equations 3.4 and 3.5, respectively (see Figure 3.2).

$$
\begin{gathered}
\Delta T c_{i, j}=T_{i, j}-T_{i, j+1} \\
\Delta d_{i, j}=d_{i, j}-d_{i, j+1}
\end{gathered}
$$


where $\Delta T c_{i, j}$ is the time delay change of $i^{\text {th }}$ boundary echo between the frame numbers $j$ and $j-1, \Delta d_{i, j}$ is the displacement of the $i^{\text {th }}$ boundary echo between the frame numbers $j$ and $j-1, j=2,3, \ldots, F$, and $F$ is the total number of frames.

Therefore, the time delay of the boundary echo is obtained by:

$$
T_{i, j}=T_{i, 1}+\sum_{k=2}^{F} \Delta T c_{i, k}
$$

Furthermore, the depth of the tissue boundary is obtained by:

$$
d_{i, j}=d_{i, 1}+\sum_{k=2}^{F} \Delta d_{i, k}
$$

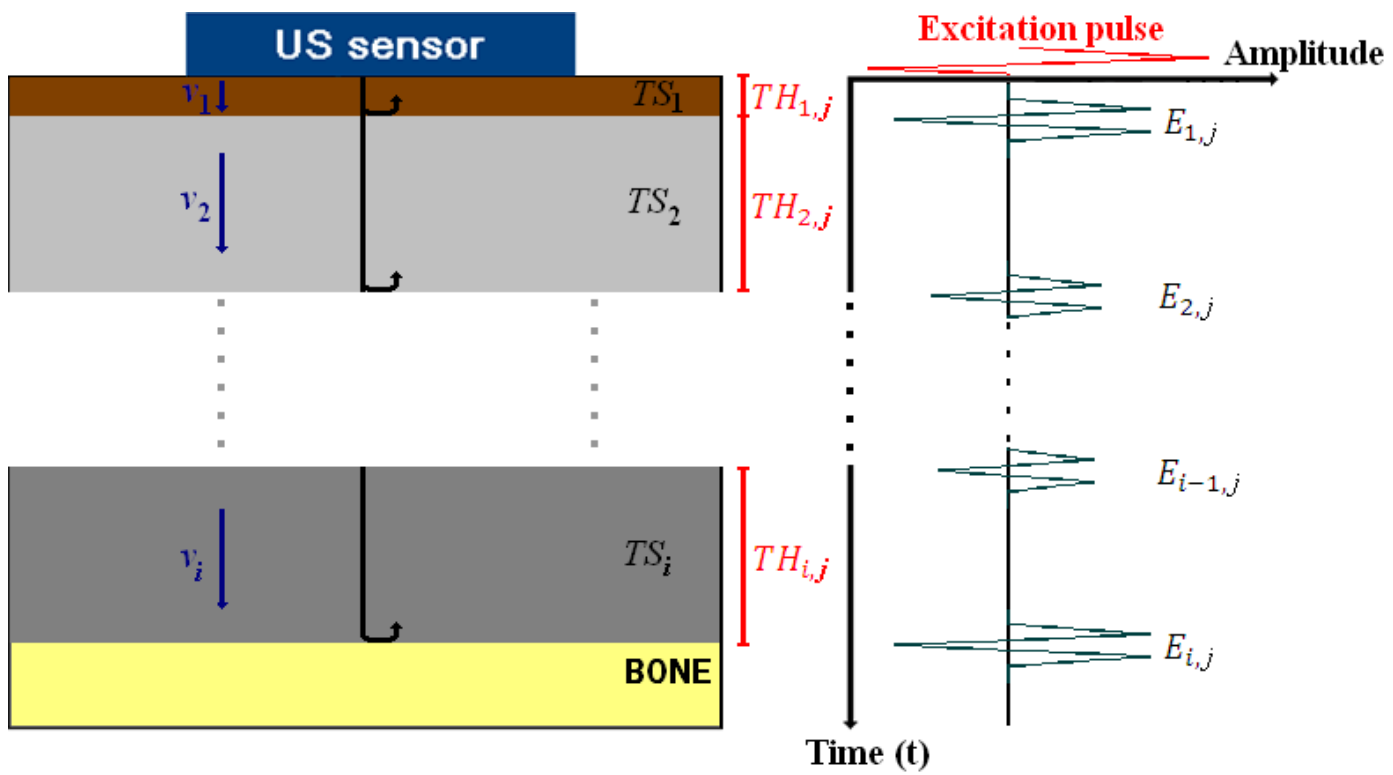

Figure 3.1: Ultrasonic pulse-echo measurement model. $T S_{i}$ denotes the $\boldsymbol{i}^{\text {th }}$ tissue layer, and $v_{i}$ is the acoustic velocity in $T S_{i}, T H_{i, j}$ is the $T S_{i}$ thickness, and $E_{i, j}$ is the boundary echo. 


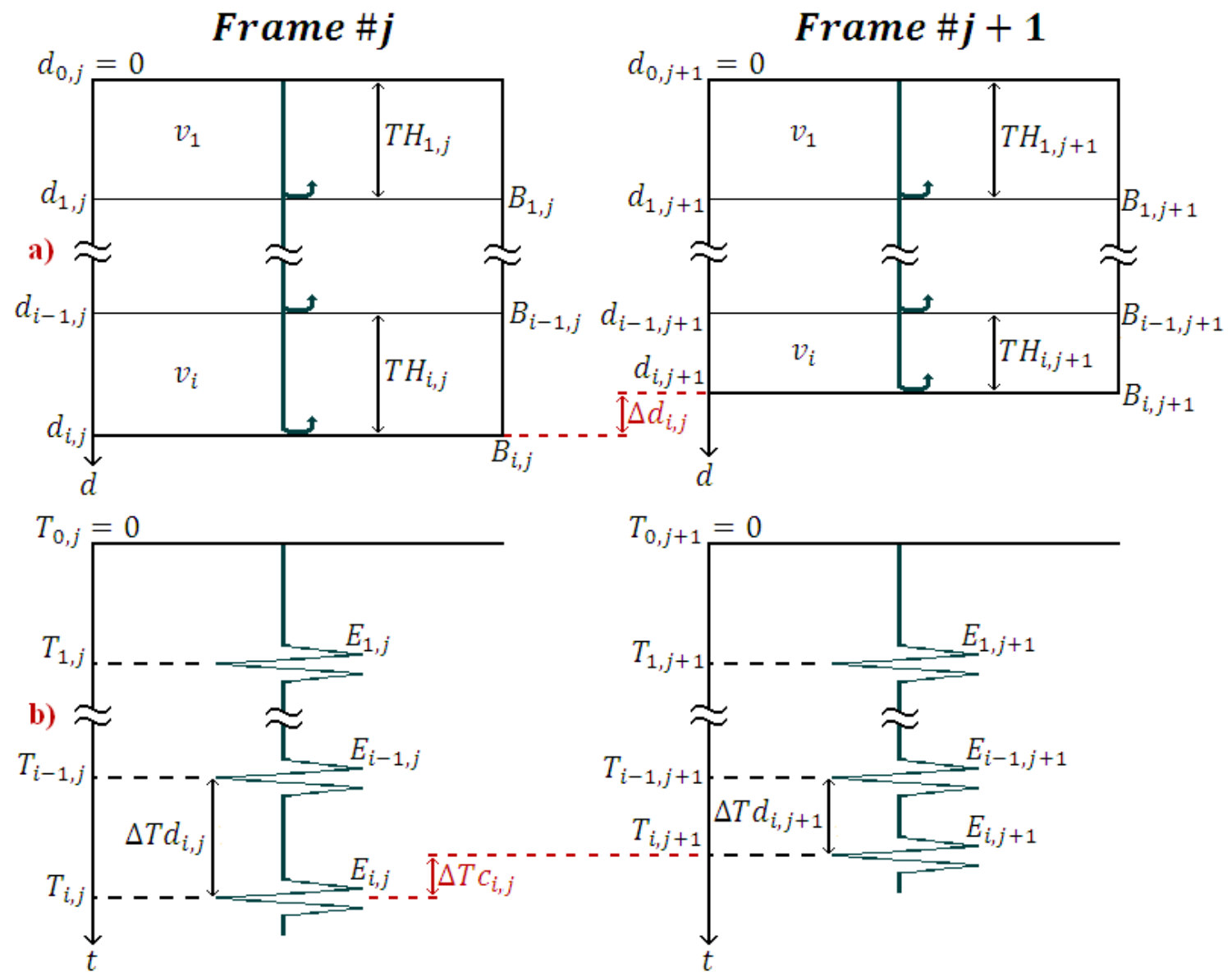

Figure 3.2: Schematic representation of two measurement frames $\boldsymbol{j}$ and $\boldsymbol{j}+1$. a) tissue boundary locations in space domain, b) tissue boundary echoes in time domain. $B_{i, j}$ denotes the tissue boundary, and $E_{i, j}$ denotes the tissue boundary echo. $d_{i, j}$ is the echo boundary $\left(B_{i, j}\right)$ depth, $T_{i, j}$ is the time delay of the boundary echo, $\Delta T d_{i, j}$ is the time delay difference between two consecutive boundary echoes in the same frame, $\Delta T \boldsymbol{C}_{i, j}$ is the time delay change of the boundary echo between two consecutive frames, and $\Delta d_{i, j}$ is the tissue boundary displacement.

\subsection{Signal Processing Techniques}

In this research, ultrasonic signals within a desired time window covering a tissue depth of interest were acquired with a fixed frame rate. The detailed signal acquisition procedure employed for the experiment will be described in Chapter 5. Due to random 
noises and undesired echoes from scatterers (scattering echoes) in tissues, detecting tissue boundary echoes is challenging using a wearable ultrasonic sensors in practice because of its relatively weak ultrasonic signal strength comparing to a conventional ultrasonic probe made with a piezoelectric ceramic. Figure 3.3 depicts the scattering echo model. These random noises and scattering echoes require us to use appropriate signal processing techniques to be able to extract the tissue boundary echoes from acquired signals and to improve the estimation accuracy of the time delay of the boundary echoes. Each boundary echo is windowed in signal processing in order to estimate the time delay of each boundary echo separately as seen in Figure 3.4. The time window is manually setup wide enough to cover the time delay changes of a selected echo but narrow enough not to cover the other echoes through the entire measurement frames. The signal processing techniques investigated in this thesis are described in the following sections.

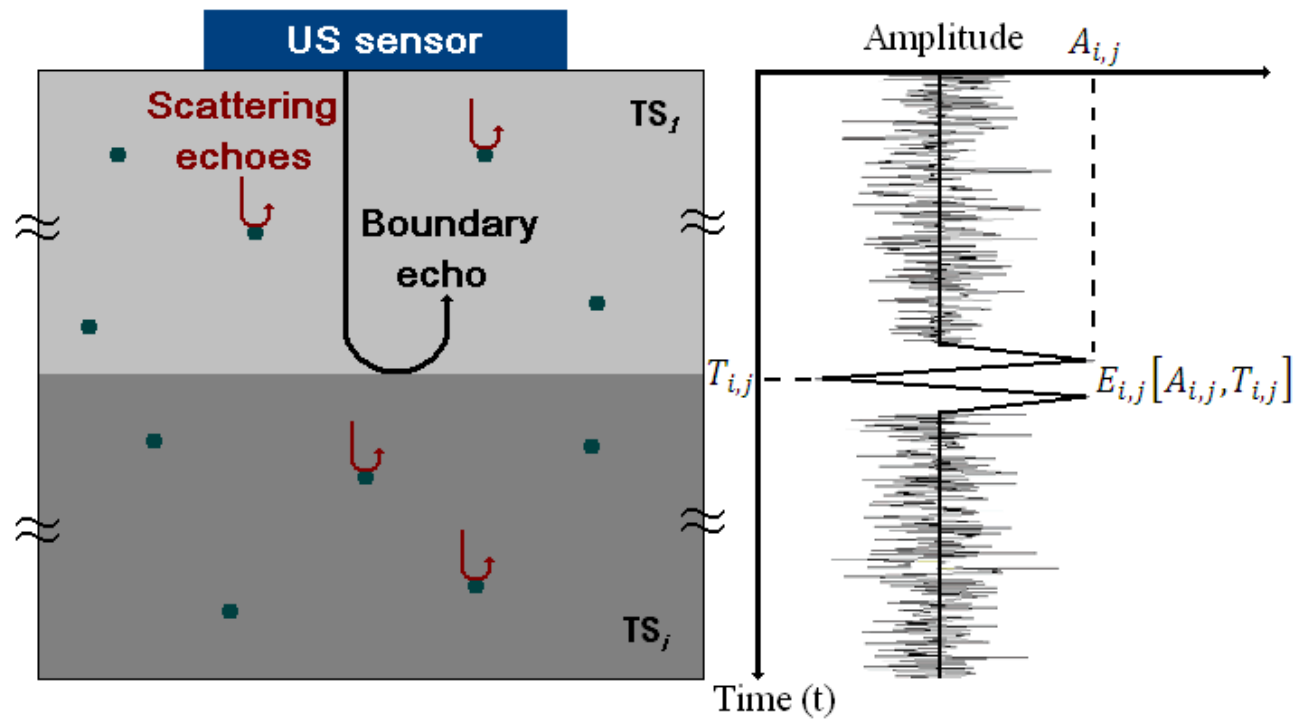

Figure 3.3: Scattering echoes model. 


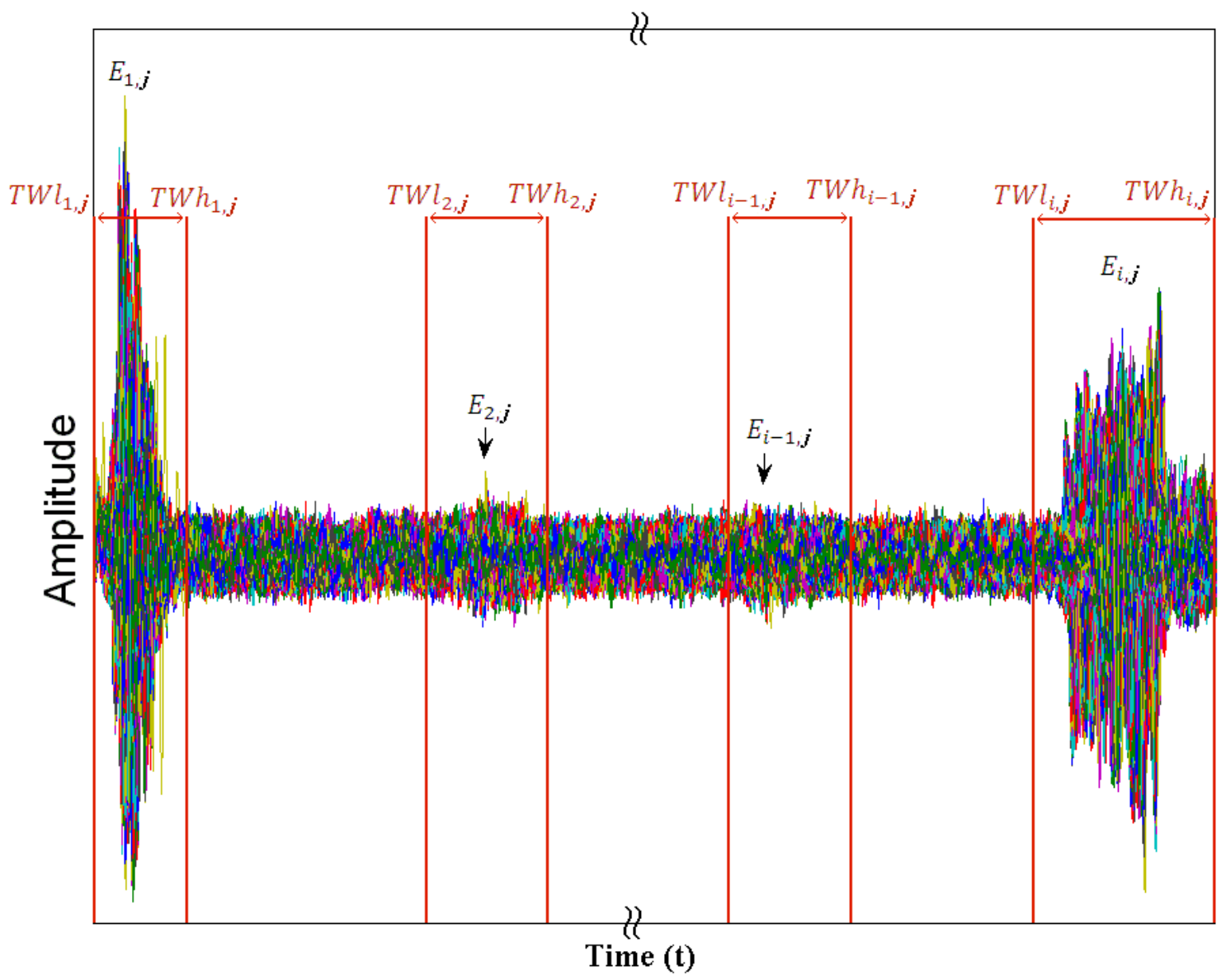

Figure 3.4: Time window setup in signal processing to detect a selected boundary echo. $T W l_{i, j}$ is the lower window limit, $T W l_{i, j}$ is the higher window limit, for the boundary echo $E_{i, j}$.

\subsubsection{Echo Peak Detection}

The time delay $\left(T_{i, j}\right)$ of an $i^{\text {th }}$ echo where the largest amplitude value (peak echo) is obtained in the reflected ultrasonic wave represents the location of the tissue boundary. Figure 3.5 demonstrates the echo peak in acquired signals. The time delay of the boundary echo is obtained by: 


$$
E_{i, j}\left[A_{i, j}, T_{i, j}\right]=\max \left[S_{i, j}(t)\right]_{t=T W l_{i, j}: T W h_{i, j}}
$$

where $E_{i, j}$ is the tissue boundary echo, $A_{i, j}$ is the amplitude of the boundary echo, $T_{i, j}$ is the time delay of the boundary echo, and $S_{i, j}(t)$ is the measured signals between the low time window limit $\left(T W l_{i, j}\right)$ and the high time window limit $\left(T W h_{i, j}\right)$ of $i^{t h}$ boundary echo. Depth of the tissue boundary can be obtained by converting the time delay $\left(T_{i, j}\right)$ obtained by Equation 3.6 to the distance $\left(d_{i, j}\right)$ using the acoustic velocity of the tissue $\left(v_{i}\right)$ by Equations 3.1 and 3.2.

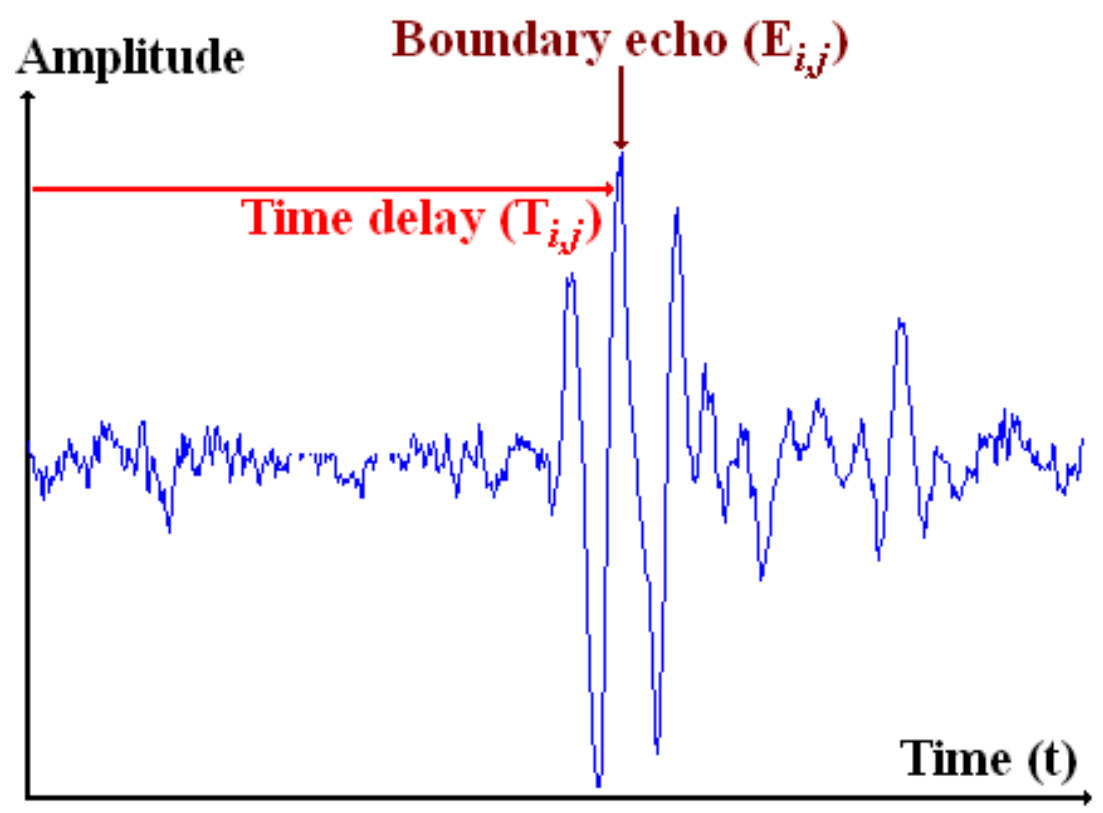

Figure 3.5: Echo peak indicates the tissue layer boundary. 


\subsubsection{Cross-correlation}

The cross-correlation technique finds the best correlation between the signals in two frames in order to determine the time lag between the signals (selected boundary echoes in our case). The time lag at the best correlation value represents the displacement of a tissue boundary after converting the time lag (time delay difference of a chosen echo between two frames) into distance using Equations 3.1, 3.2, 3.6 and 3.7. Hence, tissue thickness change can be calculated. The correlation between two frames is calculated by $[98,99]:$

$$
C\left(G_{i, j-1}\right)=\int_{T W l_{i, j-1}}^{T W h_{i, j-1}} S_{i, k}(t) \cdot S_{i, j}\left(t+G_{i, j-1}\right) d t, j=2,3, \ldots, F
$$

where $C\left(G_{i, j-1}\right)$ is the correlation between the $k^{\text {th }}$ frame and $j^{\text {th }}$ frame, $F$ is the total number of frames, and $S_{i, j}(t)$ is the $j^{t h}$ frame of the measured signals. The largest value of $C\left(G_{i, j-1}\right)$ gives the best correlation where $G_{i, j-1}=\Delta T c_{i, j-1}$. When $k=1$, the crosscorrelation between first frame and other is calculated to obtain the time delay change of $i^{\text {th }}$ echo between the first and other frames, while $k=j-1$ for the cross-correlation between two consecutive frames technique.

Due to scattering echoes and body motion in the in-vivo experiment, the boundary echo shape was changing significantly during muscle contraction. Thus, the correlations between the first frame and later frames were decreasing (the details are discussed in Chapter 5). To overcome this problem, cross-correlation between two consecutive frames 
technique was also used so that the echo shape difference between the two frames could be minimal.

\subsubsection{Echo Phase Detection}

The aim here is to obtain the tissue boundary depth using the phase of the boundary echo signals at a selected frequency (typically the center frequency of the echo). Fast Fourier Transformer was used to transform the acquired signals from the time domain to frequency domain. Then, the phase at a selected frequency is obtained (the selected frequency has the largest magnitude among all frequencies) $[92,93]$.

The obtained phase of an echo in all the frames are subtracted by the phase of the echo in the first frame as a reference:

$$
\varphi r_{i, j}=\varphi_{i, j}-\varphi_{i, 1}, j=1,2, \ldots, F
$$

where $\varphi_{i, j}$ is the unwrap phase at the chosen frequency $f c_{i}$ in each $j^{\text {th }}$ frame and $\varphi r_{i, j}$ is the unwrap phase with the first frame echo phase referenced. Finally, the unwrap phase of $i^{\text {th }}$ boundary echo at a chosen frequency can be converted to the $i^{\text {th }}$ tissue boundary depth by:

$$
\begin{gathered}
2 \cdot d r_{i, j}=\frac{\lambda_{i, j}}{2 \cdot \pi} \varphi r_{i, j} \\
d r_{i, j}=\frac{v_{i}}{4 \cdot \pi \cdot f c_{i}} \varphi r_{i, j}
\end{gathered}
$$




$$
d_{i, j}=d r_{i, j}+d_{i, 1}
$$

where $d r_{i, j}$ is the depth difference of the $i^{\text {th }}$ boundary from the depth of that in the first frame, $\lambda_{i, j}$ is the wavelength at the chosen frequency.

$d_{i, 1}$ is the depth of the $i^{\text {th }}$ boundary echo in the first frame which is obtained from the echo peak detection technique by Equation 3.1. The reflected ultrasonic signals have a frequency bandwidth since the transmitted ultrasonic wave is pulsed ultrasound but not a continuous wave having a single frequency component. The frequency bandwidth of reflected ultrasonic signals could be changed due to the attenuation and interference with scattering echoes. Taking the average of the phases at multiple frequencies around the center frequency of the desired boundary echo could improve the SNR of the phase measurement. The frequency range used for the phase averaging is between $f l_{i, j}$ and $f h_{i, j}$ centered by the chosen frequency $f c_{i}$. The averaged phase is obtained by:

$$
\operatorname{ave}_{i, j}=\frac{1}{f h_{i, j}-f l_{i, j}} \cdot \int_{f l_{i, j}}^{f h_{i, j}} \varphi_{i, j}(f) d f
$$

The tissue boundary depth is obtained from this averaged phase using the same calculation procedure given by the Equations 3.10 to 3.13. 


\subsubsection{LQ-factorization}

LQ-factorization can decompose the measured boundary echo into several frequency components in order to separate desired signals of the tissue boundary echo from undesired random noises and scattering echoes which may have different frequency components from the desired boundary echo. Recently, LQ-factorization has been used for signal processing. Two studies have used the LQ-factorization in order to separate noises from the measured signals $[94,95]$. Therefore, the LQ-factorization technique is applied in this research to extract desired signals (boundary echoes) from the measured signals. The LQ-factorization equations are represented in discrete form.

One or more frequencies can be selected from the frequency components of tissue boundary echo. Signal is generated from each selected frequency by:

$$
S f q_{i, j}(n)=A \cdot \sin \left(2 \cdot \pi \cdot n \cdot T_{s} \cdot f q_{i}+\vartheta\right), n=1,2, \ldots, N
$$

where $S f q_{i, j}(n)$ is signal generated with $q^{\text {th }}$ selected frequency, $T_{s}$ is the sampling rate, $q$ is the order number of the selected frequency, $f q_{i}$ is the selected frequency, $A$ is the signal amplitude value, $\vartheta$ is the initial phase value, $n$ is the ultrasound rf signal sample index, and $N$ is the total sample number corresponding to the tissue depth. The LQfactorization is identified by Equation 3.16. The left side of the equation is a matrix with the measurement signals $S_{i, j}(n)$ and the generated signals $S f q_{i, j}(n)$ windowed to include 
only $i^{\text {th }}$ boundary echo. The right side of the equation is the outcome from the LQfactorization processes. It contains two matrixes: $L$ and $Q$.

$$
\begin{aligned}
& {\left[\begin{array}{c}
S f 1_{i, j}(n) \\
\vdots \\
S f q_{i, j}(n) \\
S_{i, j}(n)
\end{array}\right]_{(N \times R)}=L_{(R \times R)} Q_{(N \times R)}, R=F+a} \\
& L=\left[\begin{array}{cccc}
1 & 0 & 0 & 0 \\
0 & 1 & 0 & 0 \\
\vdots & \cdots & \ddots & 0 \\
b_{1} & \cdots & b_{q} & \iota_{(F \times F)}
\end{array}\right]_{(R \times R)} \\
& Q=\left[\begin{array}{c}
S f 1_{i, j}(n) \\
\vdots \\
S f q_{i, j}(n) \\
\grave{Q}_{(N \times F)}
\end{array}\right]_{(N \times R)} \\
& S_{i, j}(n)=\left[\begin{array}{lll}
b_{1} & \cdots & b_{q}
\end{array}\right]\left[\begin{array}{c}
S f 1_{i, j}(n) \\
\vdots \\
S f q_{i, j}(n)
\end{array}\right]+\grave{L} \grave{Q}
\end{aligned}
$$

where $L$ is the lower triangular matrix of the decomposed measurement signals, $Q$ is the orthogonal unity matrix, $F$ is the total number of frames, $N$ is the total sample number corresponding to the tissue depth, $a$ is the total number of chosen frequencies to be separated, $b_{q}$ is $L$ matrix element, $\grave{L}$ is a matrix taken from $L$, and $\grave{Q}$ is a matrix taken from $Q$. The separated signal (or signals) at the chosen frequency (or frequencies) is given by:

$$
d S_{i, j}(n)=\grave{L} \grave{Q}
$$


In this research, only one frequency (most likely the center frequency) was chosen to be separated from the acquired signals in each boundary echo. Cross-correlation technique between two consecutive frames is used to obtain the time delay change of the boundary echo using $d S_{i, j}(n)$. 


\section{Chapter 4}

\section{Numerical Simulation Experiments}

In the in-vivo experiment, as we will see in Chapter 5, there are several types of signals were acquired in ultrasonic pulse-echo measurements. These include ultrasonic waves reflected from tissue boundaries in the human body, and electrical and environmental noises. The reflected ultrasonic waves are categorized into boundary echoes and scattering (or speckle) echoes. Our goal is to track the tissue boundary to measure the tissue displacement and thickness using the boundary echoes. On the other hand, the scattering echoes are considered undesired signals or noise in this study. In order to better understand the in-vivo experimental data and investigate the effectiveness, reliability, and feasibility of the signal analysis techniques descried in Section 3.2, this chapter will study how the scattering echoes affect the boundary motion tracking accuracy using numerical simulation experiments.

\subsection{Numerical Simulation Method}

\subsubsection{Boundary Echo}

Ultrasonic waves propagate through body tissues and reflect from tissue boundaries, which results in ultrasonic echoes. The boundary echoes are the reflected 
ultrasonic waves from a boundary between two different mediums (tissues in this study) because of the difference in the acoustic impedance of the two tissues [97]. A Gaussian radio frequency (rf) pulse model was used to model the boundary echoes by the following formula [100]:

$$
E_{i, j}(t)=e^{-\beta \cdot t^{2}} \cdot \cos \left(\omega c_{i} \cdot t\right), t=-T T / 2: T T / 2
$$

where $\beta$ is the bandwidth factor, TT is the total acquisition time for each frame corresponding to the tissue depth, and $\omega c_{i}$ is the center angular frequency of the boundary echo where $f c_{i}$ assumed to be $5 \mathrm{MHz}$ (see Section 4.1.6).

Similarly, Equation 4.1 can also be defined in discrete form by:

$$
E_{i, j}(n)=e^{-\beta \cdot\left(n \cdot T_{S}\right)^{2}} \cdot \cos \left(\omega c_{i} \cdot n \cdot T_{s}\right), n=-N / 2: N / 2
$$

where $\mathrm{N}$ is the total number of sample points corresponding to the tissue depth and $T_{S}$ is the sampling period. An example of a simulated boundary echo is presented in Figure 4.1. 


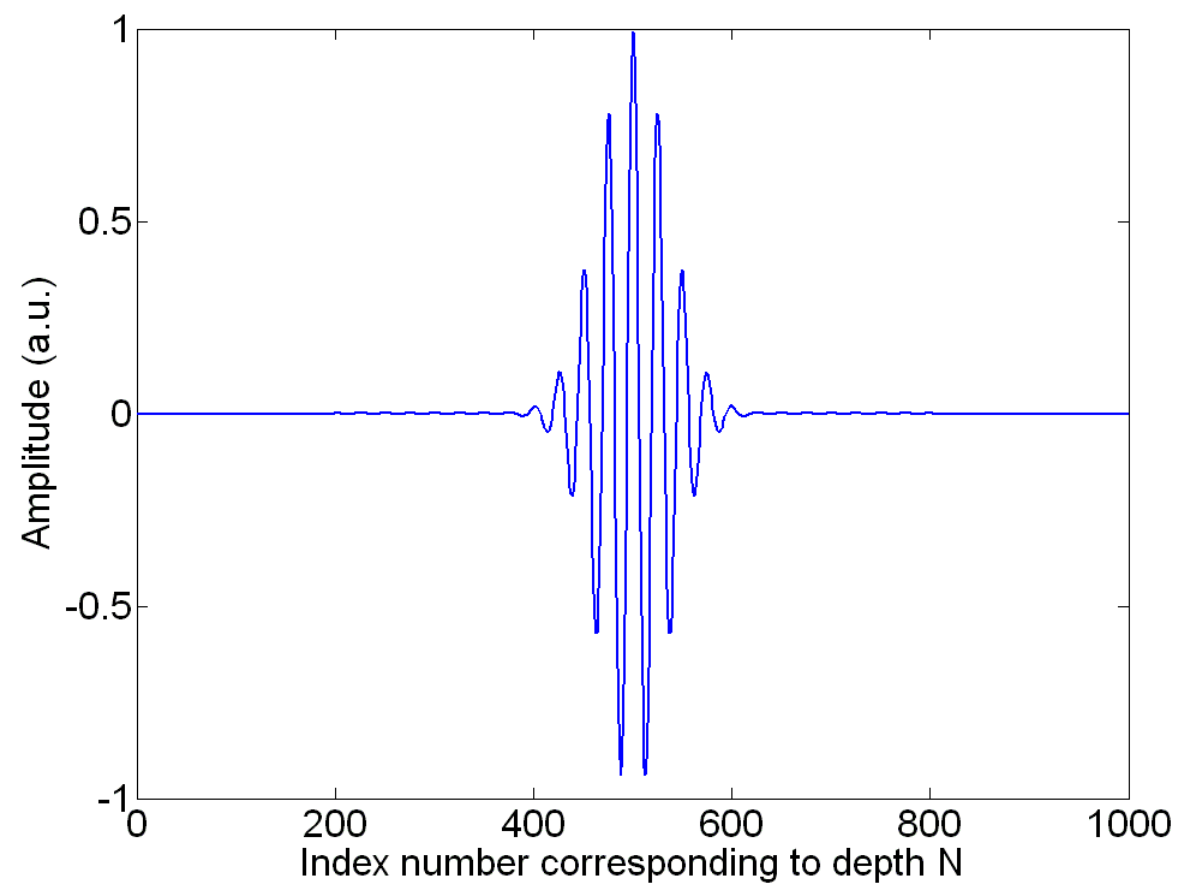

Figure 4.1: Simulated Gaussian rf pulse echo which represents the tissues boundary echo.

\subsubsection{Scattering Echoes}

Since the scattering echoes are considered as a main noise source (undesired signals), random noises are not simulated in this study. Therefore, the term "noise" will refer to scattering echoes hereafter. The scattering particles in the body are the causes of the scattering echoes that blur the ultrasonic images.

The scattering echoes produced by Gaussian rf pulse signals defined in Equation 4.1 can be modeled by: 


$$
\begin{gathered}
s E_{m, j}\left(\tau_{m, j} ; t\right)=\alpha_{m, j} \cdot E_{i, j}\left(t-\tau_{m, j}\right) \\
=\alpha_{m, j} \cdot e^{-\beta\left(t-\tau_{m, j}\right)^{2}} \cdot \cos \left(\omega c_{i} t-\omega c_{i} \tau_{m, j}\right), m=1,2, \ldots, J
\end{gathered}
$$

where $s E_{m, j}\left(\tau_{m, j} ; t\right)$ is a signal scattering echo from $m^{t h}$ scatterer, $\alpha_{m, j}$ is the amplitude factor, $J$ is the total number of scatterers within the ultrasonic beam, and $\tau_{m, j}$ is the time delay between the ultrasonic sensor and $m^{\text {th }}$ scatterer $[100,101] . \tau_{m, j}$ results in a phase shift in the received scattering echo. Therefore, Equation 4.3 can be rewritten as:

$$
s E_{m, j}\left(\tau_{m, j} ; t\right)=A_{m, j}(t) \cdot \cos \left(\omega c_{i} t-\theta_{m, j}\right)
$$

where $A_{m, j}(t)=\alpha_{m, j} \cdot e^{-\beta\left(t-\tau_{m, j}\right)^{2}}$ which represents the amplitude and $\theta_{m, j}=\omega c_{i, j}$. $\tau_{m, j}$ represents the phase of $m^{t h}$ scattering echo.

The time delay $\tau_{m, j}$ of an echo propagating between the ultrasonic sensor and $m^{t h}$ scatterer in the ultrasonic pulse-echo method can be found by:

$$
\tau_{m, j}=\frac{2 \cdot D_{m, j}}{v_{i}}
$$

where $D_{m, j}$ is the distance between the ultrasonic sensor and the $m^{\text {th }}$ scatterer, and $v_{i}$ is the velocity of the ultrasonic waves propagating in tissues.

Therefore, the received scattering echoes from all the scatterers are represented by:

$$
s E r_{m, j}(t)=\sum_{m=1}^{J} s E_{m, j}\left(\tau_{m, j} ; t\right)=\sum_{m=1}^{J} A_{m, j}(t) \cdot \cos \left(\omega c_{i} t-\theta_{m, j}\right)
$$

By converting Equation 4.6 into a discrete form we obtain: 


$$
s E r_{m, j}(n)=\sum_{m=1}^{J} A_{m, j}(n) \cdot \cos \left(\omega c_{i} \cdot n \cdot T_{s}-\theta_{m, j}\right)
$$

Figure 4.2 presents the simulated scattering echoes.

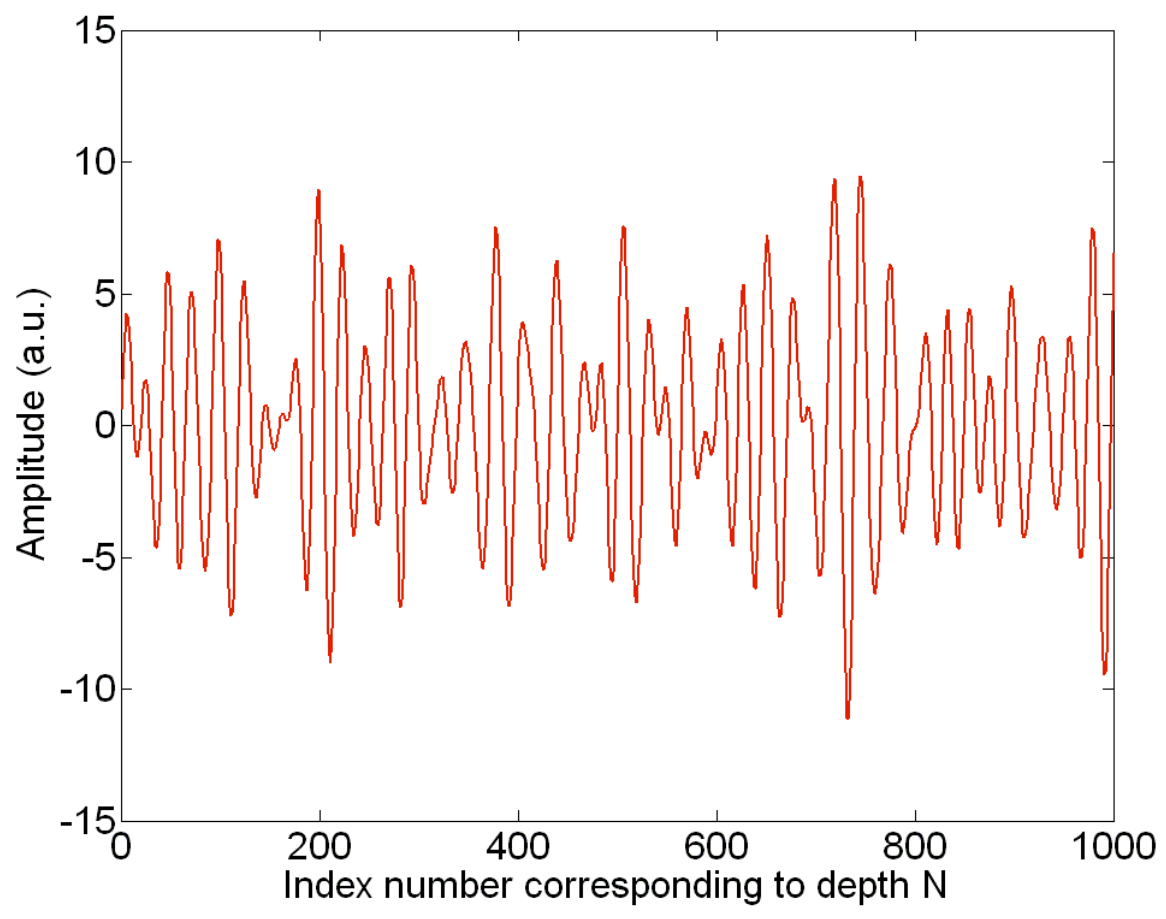

Figure 4.2: Simulated scattering echoes.

\subsubsection{Signal-to-Noise Ratio (SNR)}

The mathematical simulation for each frame in the simulation experiments can be represented by summing Equation 4.1 (the Gaussian rf pulse echo) with Equation 4.3 (the received scattering echoes) as follows: 


$$
\begin{gathered}
s S_{i, j}(t)=E_{i, j}(t)+s E r_{m, j}(t) \\
=e^{-\beta \cdot t^{2}} \cdot \cos \left(\omega c_{i} \cdot t\right)+\sum_{m=1}^{J} \alpha_{m, j} \cdot e^{-\beta\left(t-\tau_{m, j}\right)^{2}} \cdot \cos \left(\omega c_{i} t-\theta_{m, j}\right)
\end{gathered}
$$

where $s S_{i, j}(t)$ is the simulated signals.

Simulated boundary echoes were tracked in different values of SNR. This step is to investigate how the noises (scattering echoes) affect the tracking accuracy of the boundary echoes for each signal analysis technique described in Section 3.2. It is important to determine the noise level in the ultrasonic signals in relation with the desired reflected echoes. SNR can be defined by:

$$
S N R=20 \cdot \log _{10} \frac{\hat{A}_{b}}{\bar{A}_{s}}
$$

where $\hat{A}_{b}$ is the maximum of the absolute value of the boundary echo, and $\bar{A}_{s}$ is the average absolute value of the scattering echoes (Figure 4.3). 


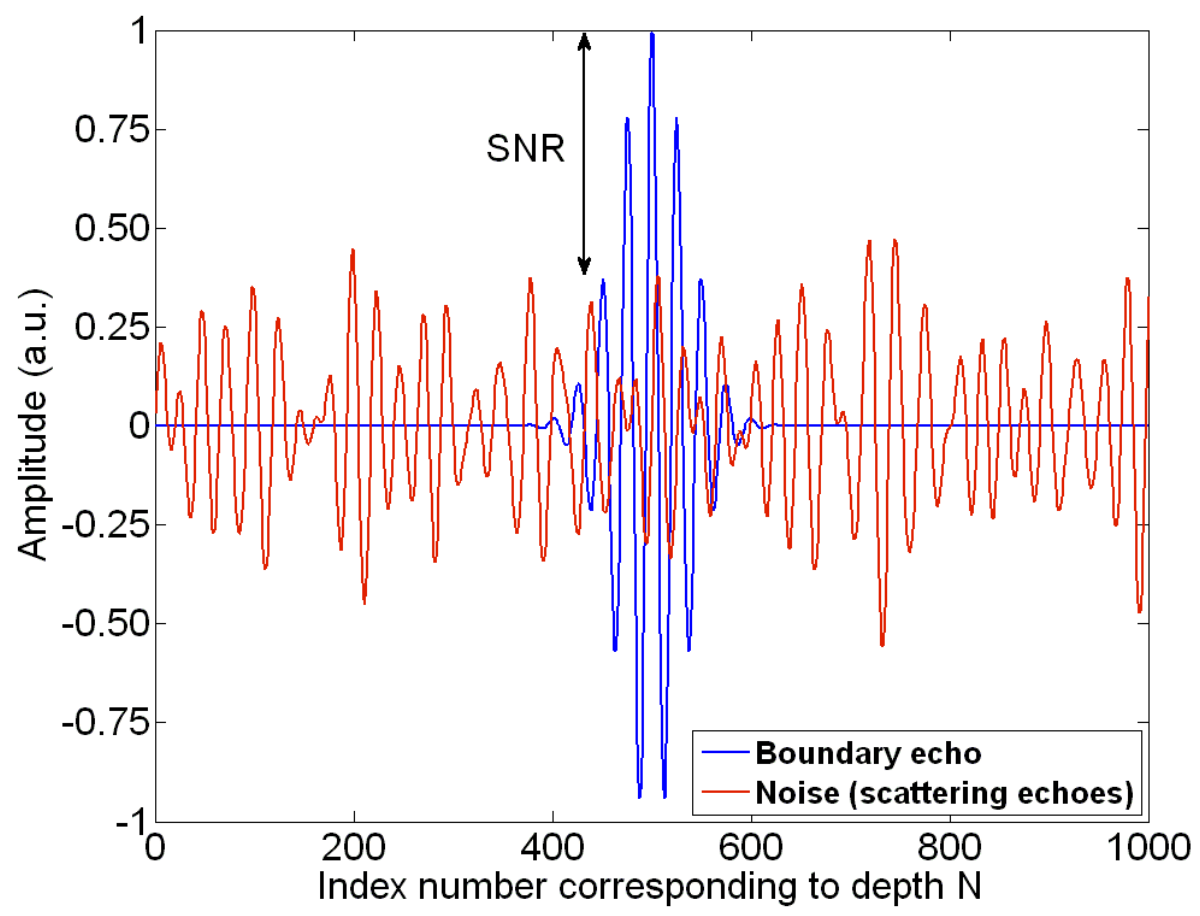

Figure 4.3: The SNR of the simulated data (Gaussian rf pulse echo + scattering echoes).

\subsubsection{Tissue Boundary Motion}

The objective of this study is to track the tissue boundary motion and tissue layer thickness changes during muscle contraction using ultrasound. As mentioned in Chapter 2, muscle thickness increases during contraction and decreases in relaxation. Tissue boundary displacement is simulated as one cycle of a sinusoidal shape in the period of the total frames. Thus, the boundary depth is given by:

$$
S M=S M_{0}+A_{N} \cdot \sin (2 \cdot \pi \cdot f m \cdot j), j=1,2, \ldots, F
$$


where $S M$ is the simulated tissue boundary depth with the sinusoidal displacement, $S M_{0}$ is the initial boundary depth, $A_{N}$ is the magnitude of displacement, $f m$ is the frequency of $S M$ signal, and $F$ is the total number of frames. Figure 4.4 shows the sinusoidal boundary echo motion. 


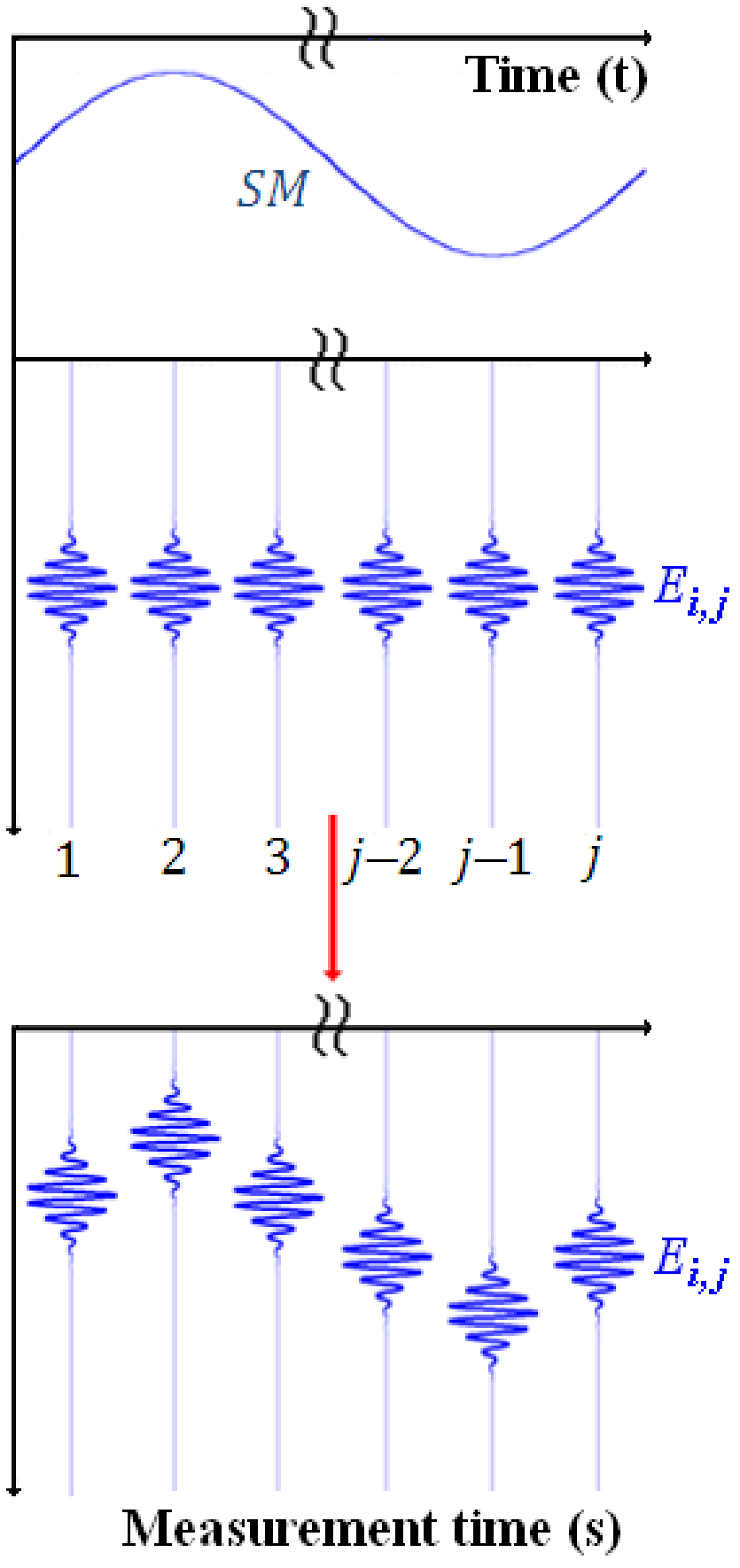

Figure 4.4: Sinusoidal boundary echo motion. The Gaussian rf pulses are shifted by the value of sinusoidal signal in each point sample. 


\subsubsection{Root Mean Squared Error (RMSE)}

The errors of boundary echo tracking for signal analysis techniques described in Chapter 3 were compared by using the RMSE values. The RMSE is defined by [102, 103]:

$$
R M S E=\sqrt{\frac{\sum_{j=1}^{F}\left(M T_{j}-\widehat{M T}_{j}\right)^{2}}{N}}
$$

where $M T_{j}$ is the boundary displacement value obtained in the simulation experiments,

and $\widehat{M T}_{j}$ is the theoretical value (model) given by Equation 4.10 for the simulations with sinusoidal motion, $j$ is the frame number, $i$ is the boundary order, and $F$ is the total number of frames.

\subsubsection{Parameter Setting For Numerical Simulation}

Tissue boundary echoes with the boundary motion having different SNR values were simulated. The signal processing techniques described in the Section 3.2 were applied to obtain the boundary displacement.

The signal acquisition conditions and parameters employed in in-vivo experiments were used to simulate the boundary echoes and motion in the numerical simulations experiments. Boundary echoes in the in-vivo experiment were found to have center 
frequency $\left(f c_{i}\right)$ of $5 \mathrm{MHz}$ with a bandwidth factor $(\beta)$ of $0.025 \mathrm{MHz}^{2}$. Sampling points of the depth $(N)$ was 1000 points in the simulation experiments, corresponding to the time window of $8 \mu \mathrm{sec}$ at the sampling frequency of $125 \mathrm{MHz}$ (or the depth of $6.52 \mathrm{~mm}$, with the ultrasonic velocity of $1630 \mathrm{~m} / \mathrm{s}$ ). The total frame number was 100 frames, corresponding to the acquisition time of $10 \mathrm{sec}$ at the frame rate of $10 \mathrm{~Hz}$. In motion simulations, one cycle of the sinusoidal motion was simulated in the entire simulated time as shown in Figure 4.4.

\subsection{Simulation experiment results}

In this section, three types of simulations were conducted: no boundary motion with random scattering echoes, sinusoidal boundary motion with fixed scattering echoes, and sinusoidal boundary motion with random scattering echoes. The SNR values employed in the simulated echoes were $0,5,10,15,20,25$ and $30 \mathrm{~dB}$. These SNR values were chosen to cover the cases when the noise amplitude is larger than the boundary echo amplitude. Then, the four signal analysis techniques (peak detection, cross-correlation, phase, and LQ factorization) presented in Chapter 3 were applied for ultrasonic signals simulated. Finally, results were compared among the techniques in each simulation to find the displacement estimation accuracy using the RMSE values. 


\subsubsection{No Boundary Motion with Random Scattering Echoes}

\subsubsection{Simulation Model}

Gaussian rf pulse echo was simulated with random scattering echoes to test the noise effects on the boundary echo detection when there is no boundary displacement. The random scattering echoes were generated with random scatterer distribution for each frame. Figure 4.5 presents this simulation model. Signals were simulated with seven different SNR values using Equation 4.10 with $S M_{0}=3.26 \mathrm{~mm}$ and $A_{N}=0$ for no boundary displacement. Figures 4.6 and 4.7 show the simulated signals of all 100 frames in the case of $30 \mathrm{~dB}$ SNR as an example in M-mode and A-mode format, respectively.

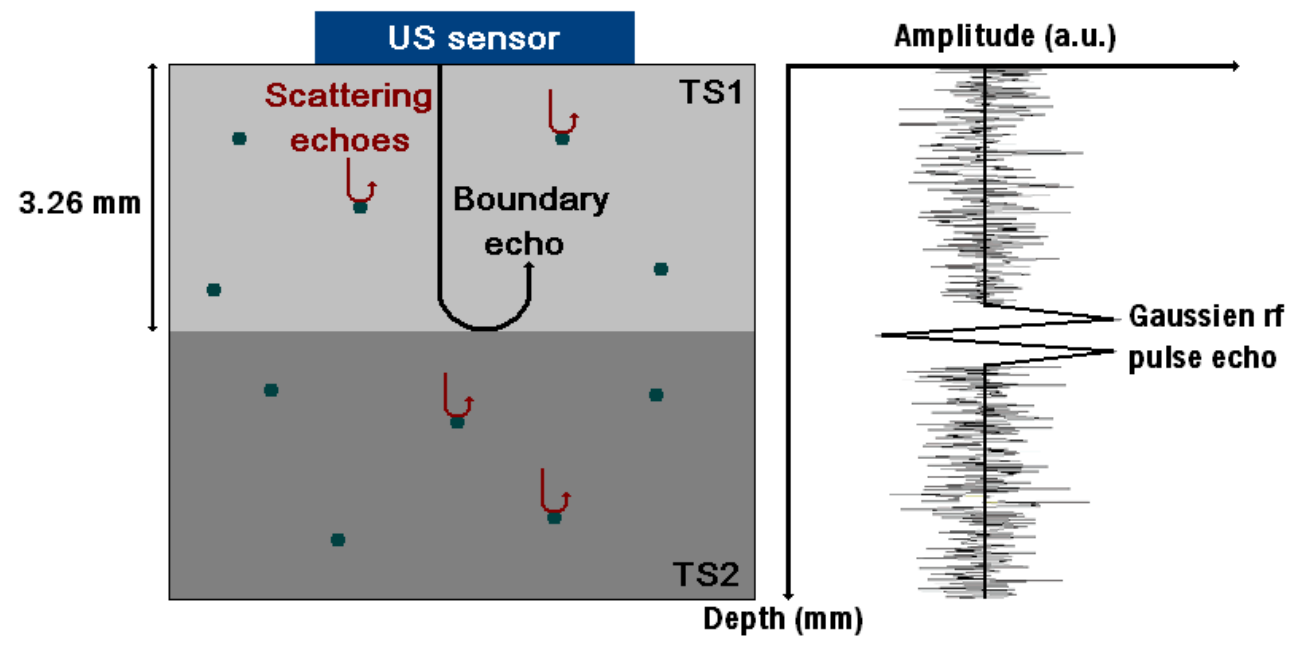

Figure 4.5: The simulation model for the no displace of tissue boundary between two tissue layers $\left(T S_{1}\right.$ and $\left.T S_{2}\right)$ with the scattering echoes. 


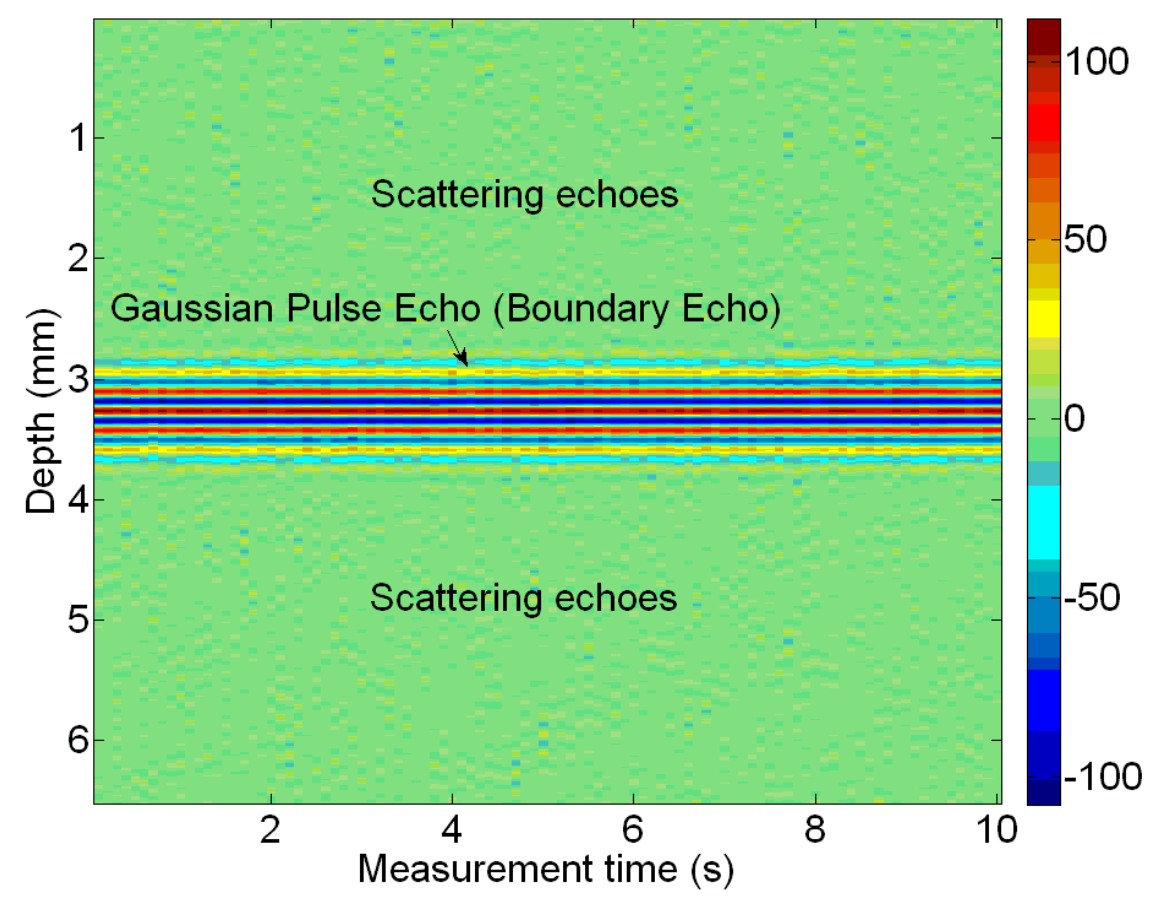

Figure 4.6: All 100 frames ( $10 \mathrm{sec}$ at $10 \mathrm{~Hz}$ frame rate) of simulated boundary echoes with no boundary motion having random scattering echoes with $30 \mathrm{~dB}$ SNR in M-mode format. 


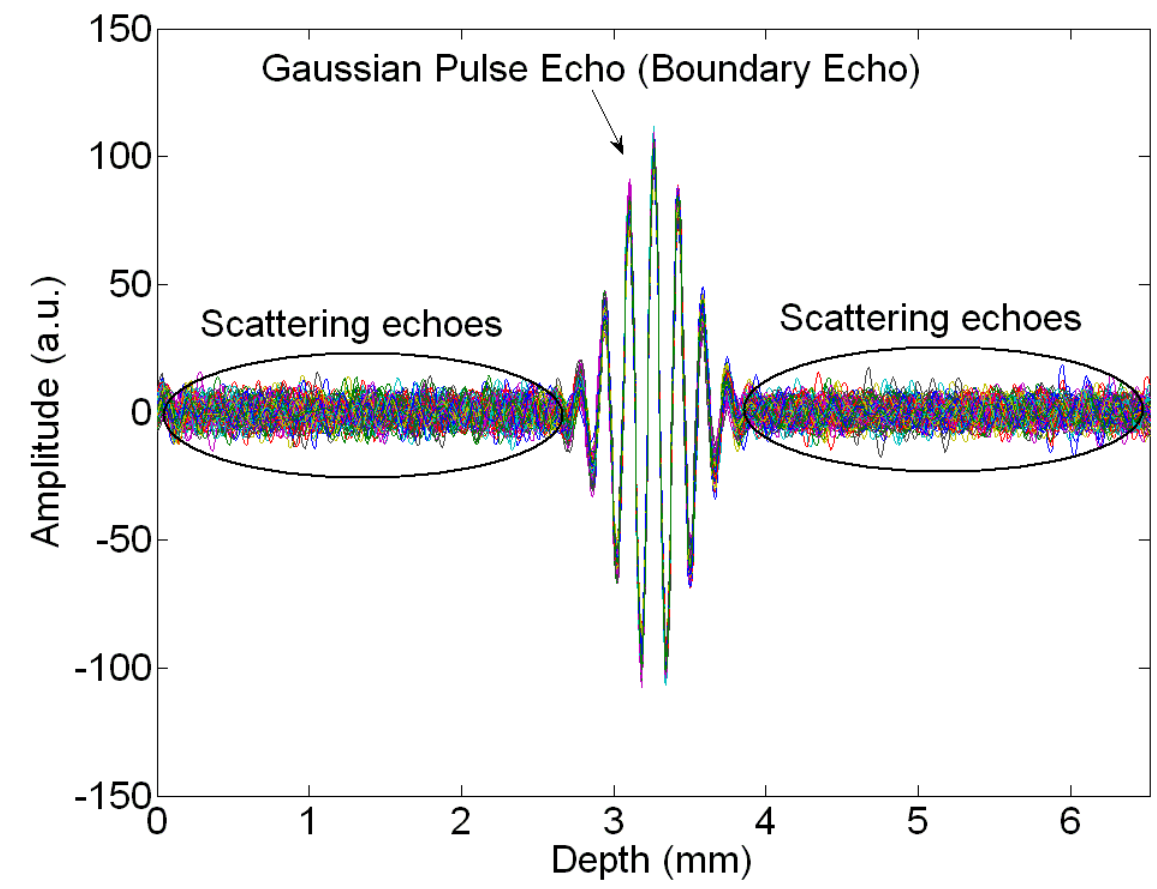

Figure 4.7: All frames of simulated boundary echoes with no boundary motion having random scattering echoes with $30 \mathrm{~dB}$ SNR in A-mode format.

The boundary echo is located at $3.26 \mathrm{~mm}$, around the middle of the total depth of $6.52 \mathrm{~mm}$. As see in Figures 4.6 and 4.7, the boundary echo is fixed and the scattering echoes are random among the frames.

Figure 4.8 shows the simulated signals of $0,5,10,15,20,25 \mathrm{~dB}$ SNR in the Mmode format. In $25 \mathrm{~dB}$ SNR (a), the boundary echo is clearly seen in red/blue colour. When the SNR value decreases below $10 \mathrm{~dB}$, the boundary echo becomes unclear and almost unobservable at $5 \mathrm{~dB}(\mathrm{e})$ and $0 \mathrm{~dB}(\mathrm{f})$. 

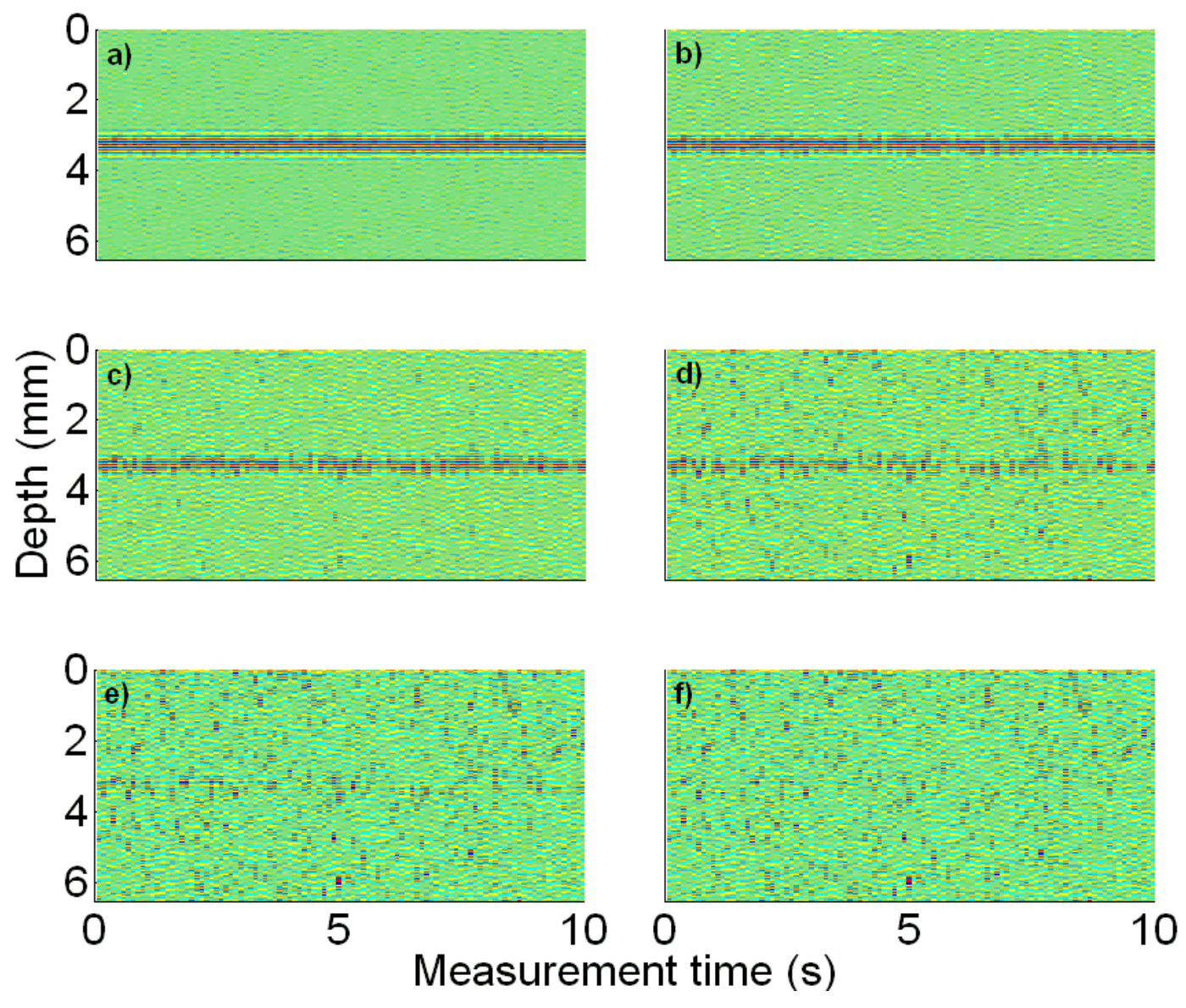

Figure 4.8: No motion with random scattering echoes simulation M-mode format (a: $25 \mathrm{~dB}$, b: $20 \mathrm{~dB}$, c: $15 \mathrm{~dB}, \mathrm{~d}: 10 \mathrm{~dB}$, e: $5 \mathrm{~dB}$, and f: $0 \mathrm{~dB}$ ).

Figure 4.9 shows the influence of the scattering echoes to the boundary echo with two different SNRs of $30 \mathrm{~dB}$ and $10 \mathrm{~dB}$. 

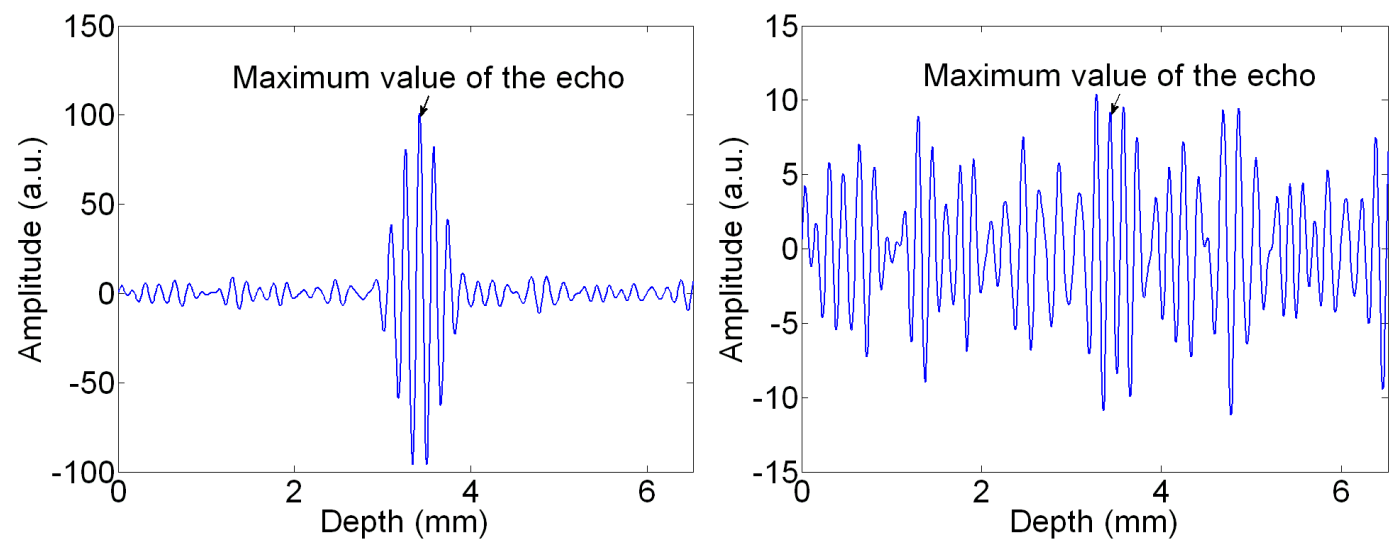

Figure 4.9: Gaussian rf pulse boundary echo with scattering echoes in $30 \mathrm{~dB}$ SNR (left), and $10 \mathrm{~dB}$ SNR (right).

\subsubsection{Displacement Estimation Results}

The signal analysis techniques mentioned in Chapter 3 were applied to this simulation experiment to determine the boundary location. The results is shown in Figure 4.10 (a: echo peak detection, b: cross-correlation between first frame and all frames, c: cross-correlation between two consecutive frames, $d$ : single frequency phase detection, e: mean of narrow band frequencies phase detection, and f: LQ-factorization). The frequency chosen for the techniques of single frequency phase detection, mean of narrow band frequencies phase detection, and LQ-factorization was the center frequency of simulated Gaussian rf pulse echoes $\left(f c_{i}=5 \mathrm{MHz}\right)$. In the mean of narrow band frequencies phase detection, ten frequencies were chosen before and after the center frequency, i.e. 4.38, 4.51, 4.63, 4.76, 4.88, 5.00, 5.14, 5.26, 5.39, 5.51, and 5.64 MHz. 

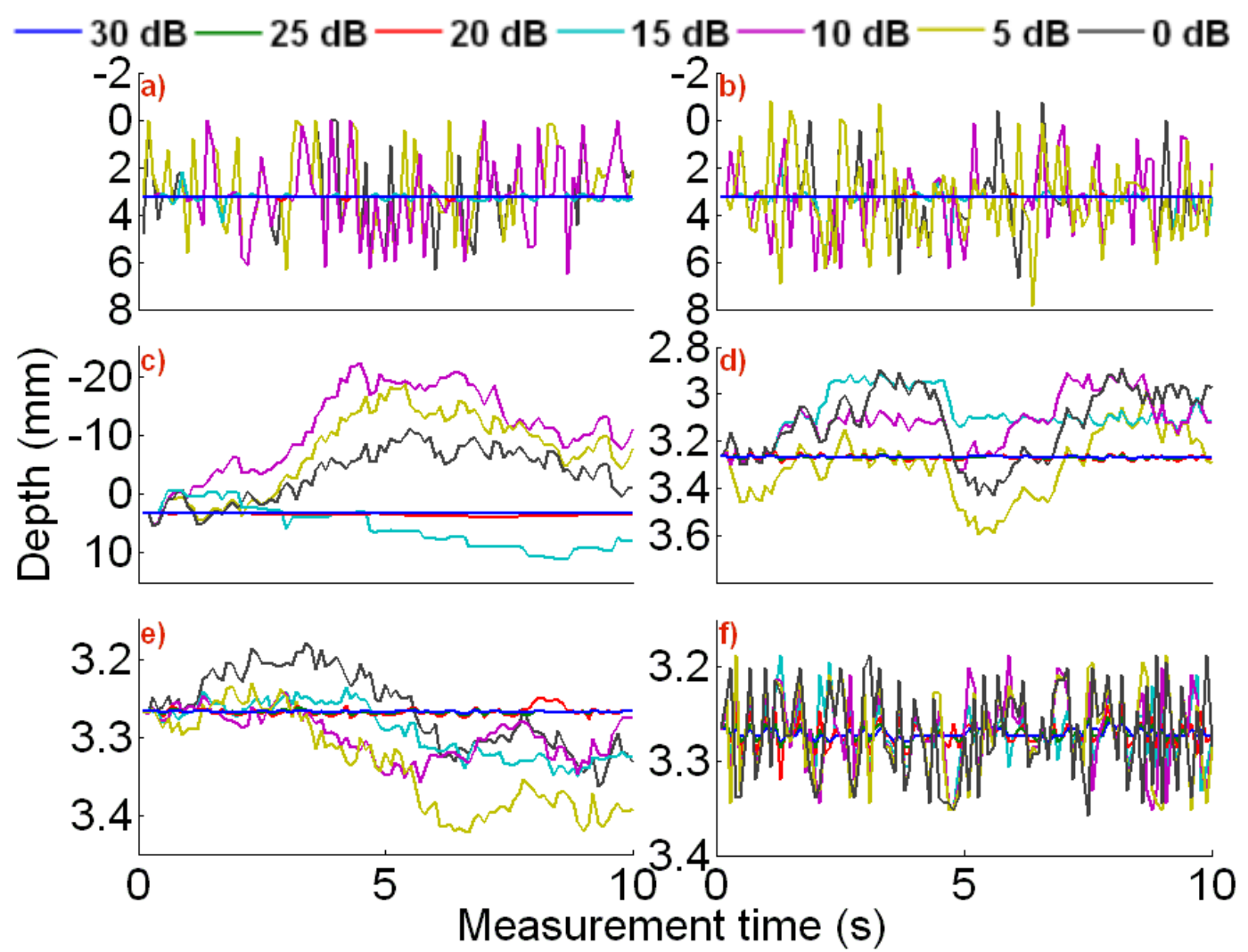

Figure 4.10: Boundary track of each technique in seven different SNR values of $30,25,20,15,10,5$, and $0 \mathrm{~dB}$ (a: echo peak detection, b: cross-correlation between firs frame and all frames, c: cross-correlation between two consecutive frames, $\mathrm{d}$ : single frequency phase detection, e: mean of narrow band frequencies phase detection, and f: LQ-factorization).

As it is observed in Figure 4.10, in general, all techniques had successfully tracked the boundary echo with $20 \mathrm{~dB}$ and larger SNR, where the errors are less than 0.1 $\mathrm{mm}$. However, for all the techniques, tracking the echo boundary had more errors with 15 $\mathrm{dB}$ and lower SNR values. In Figures 4.11 and 4.12, the RMSE values with respect to the SNR were presented. In Figures 4.10a, b, and f, random changes (or sudden jumps) of the obtained depth are observed, while in Figures 4.10c, d, and e, gradual changes of the obtained depths are observed. It was observed in Figure 4.10c that with a technique of cross-correlation between two consecutive frames, the depth of the boundary echo changed from $3.26 \mathrm{~mm}$ to beyond $-20 \mathrm{~mm}$, thus the error was more than $23 \mathrm{~mm}$. This 
large error happened because this technique started to track other echoes such as scattering echoes or noises. Moreover, errors from each cross-correlation between two consecutive frames process are accumulating. This differs from the cross-correlation between first frame and all frames technique, which had its first frame data fixed in all cross-correlation processes.

The simulated signals were also analysed using the absolute values of the signal amplitude for the following signal processing techniques in time domain: echo peak detection, cross-correlation between first frame and all frames, and cross-correlation between two consecutive frames. Results are discussed using RMSE values in the next section. Figure 4.11 is the comparison between applying the time-domain techniques on the raw signals (left) and on the signal absolute value (right). 


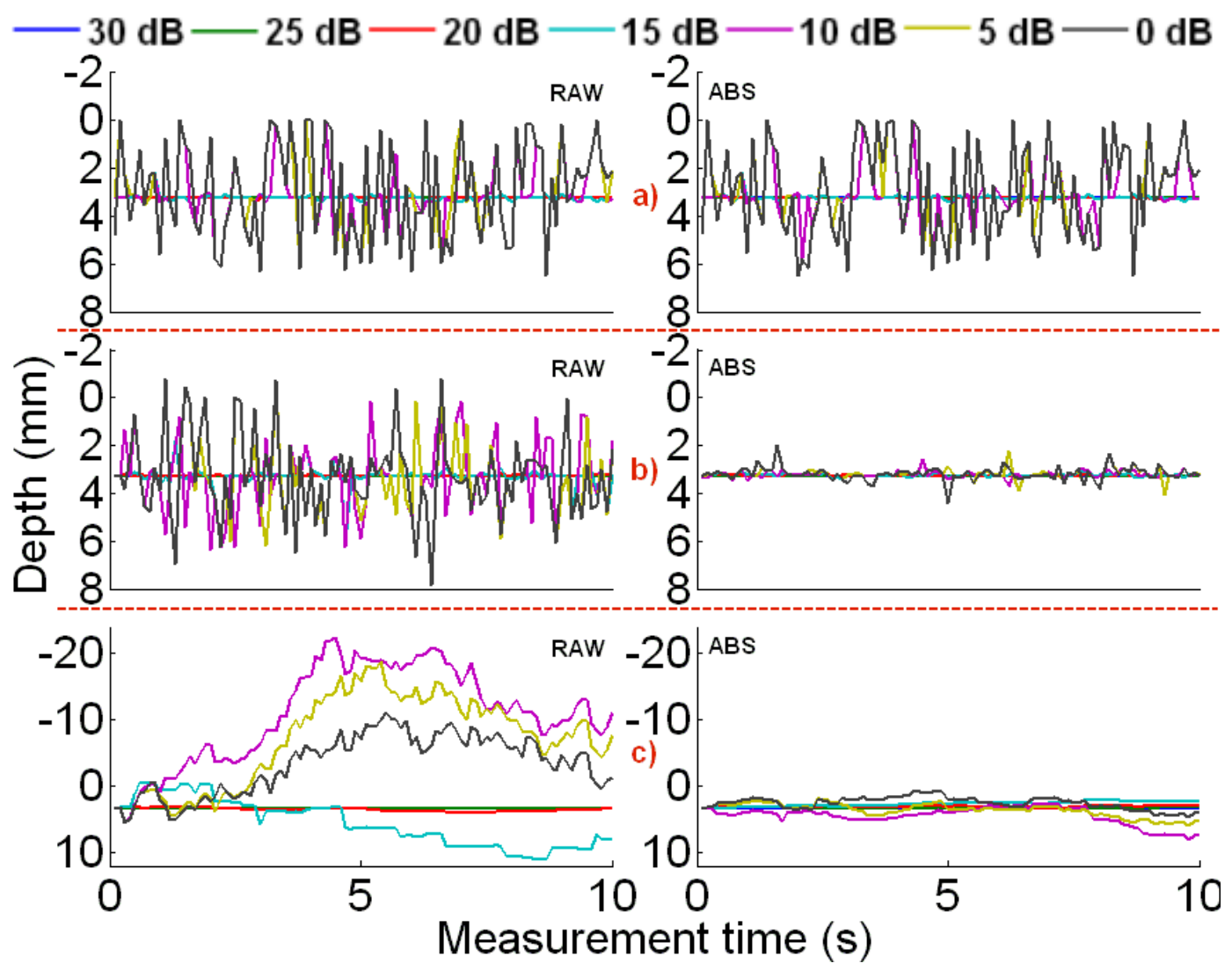

Figure 4.11: The comparisons between applying the techniques on raw signals and on signals absolute value for boundary depth estimation for simulation 1 where a) echo peak detection, b) cross-correlation between first frame and all frames, and c) cross-correlation between two consecutive frames.

\subsubsection{Root Mean Squared Error (RMSE)}

RMSE values were obtained from the results shown in Figures 4.10 and Figure A.1 in appendix A to compare the techniques with respect to the SNR values. The results are presented in Figures 4.12 and 4.13, where A denotes the echo peak detection technique, S: cross-correlation between first frame and all frames, F: cross-correlation between two consecutive frames, P: single frequency phase detection, MP: mean of 
narrow band frequencies phase detection, LQ: LQ-factorization, and ABS: absolute value of the signal amplitude with the techniques of $\mathrm{A}, \mathrm{S}$, and F.

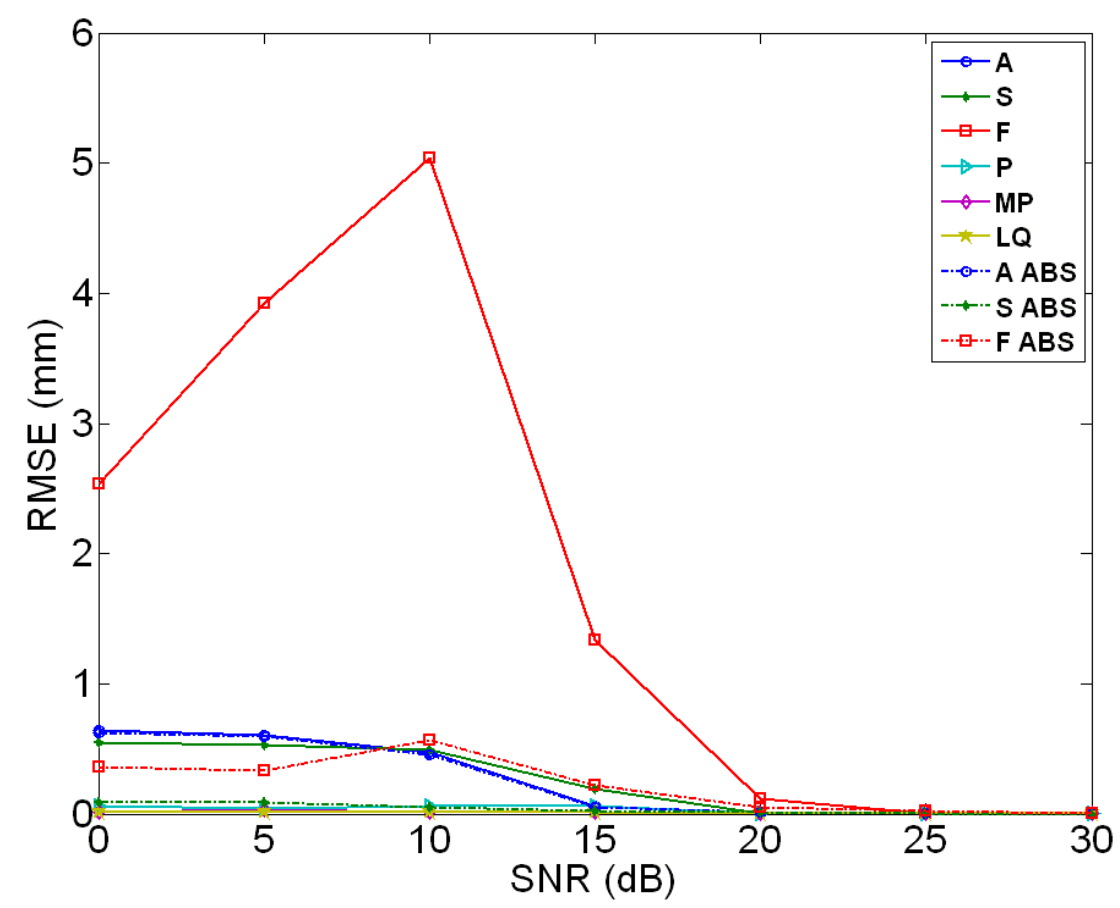

Figure 4.12: RMSE values of all techniques including the absolute value analysis for $\mathrm{A}, \mathrm{S}$ and $\mathrm{F}$ in 30, 25, 20, 15, 10, 5, and $\mathrm{O} \mathrm{dB}$ SNR (A: echo peak detection, $\mathrm{S}$ : cross-correlation between first frame and all frames, $\mathrm{F}$ : cross-correlation between two consecutive frames, P: single frequency phase detection, MP: mean of narrow band frequencies phase detection, LQ: LQ-factorization, and ABS: absolute value). 


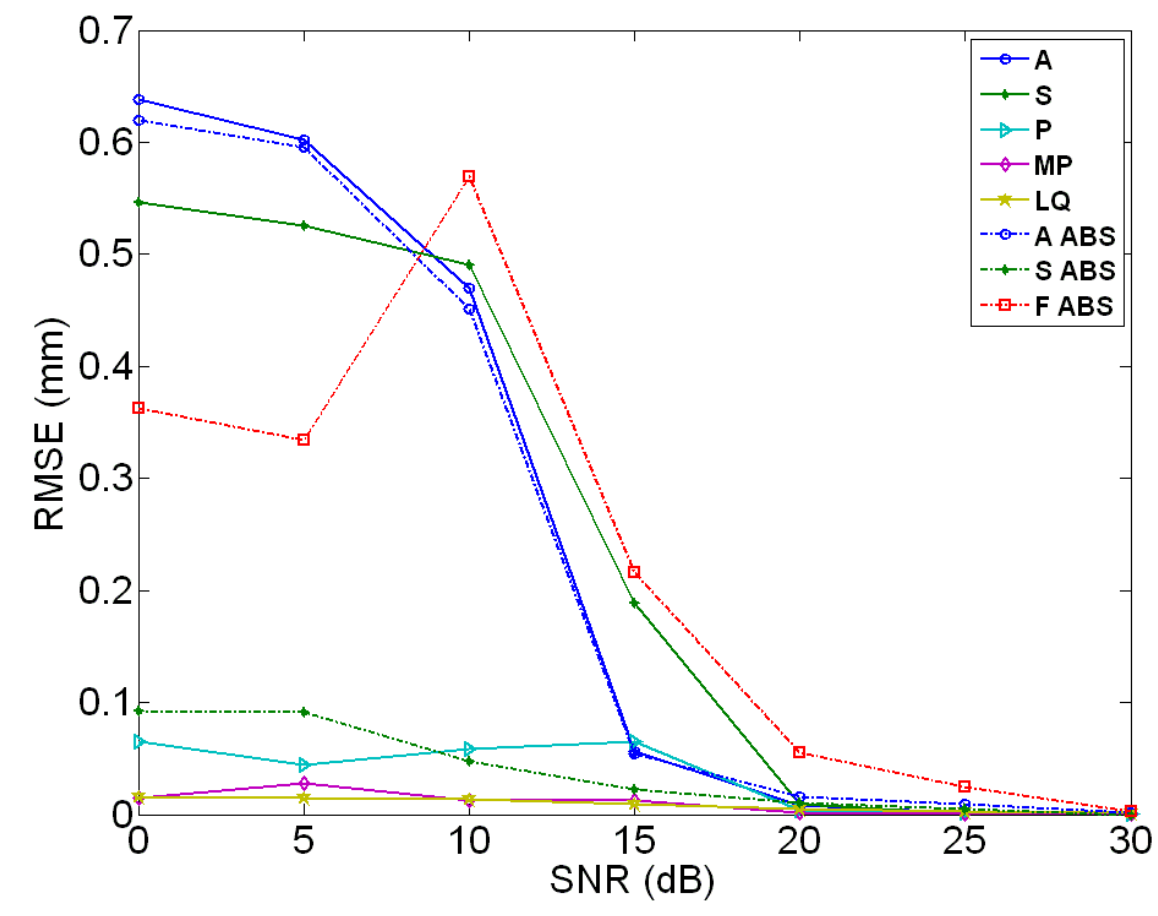

\section{Figure 4.13: The same results in Figure 4.11 but without the result of the cross- correlation between two consecutive frames $(F)$.}

From Figures 4.12 and 4.13, the overall trend is that RMSE decreases as the SNR increases. However, the values of RMSE by the techniques of F ABS, P and MP had fluctuated values. This may be because of the amplitude of the scattering echoes being occasionally larger than that of the boundary echo in low SNR. The scattering echoes would therefore be detected instead of the boundary echo.

Figure 4.12 shows a closer view of the results in Figure 4.10 without the results obtained by F which had the largest RMSE values. The three techniques in frequency domain analysis, such as S: single frequency phase detection, MP: mean of narrow band frequencies phase detection, and LQ: LQ-factorization, had better tracking accuracy compared to the other three techniques in time domain analysis such as A: echo peak detection, S: cross-correlation between first frame and all frames, and F: cross-correlation 
between two consecutive frames. In addition, the absolute value of the signals with the techniques of $\mathrm{A}, \mathrm{S}$ and $\mathrm{F}$ improved the accuracy. Using the absolute signal amplitude, the RMSE at the $0 \mathrm{~dB}, 5 \mathrm{~dB}, 10 \mathrm{~dB}$, and $15 \mathrm{~dB}$ SNR in the technique of $\mathrm{F}$ decreased from 2.5 $\mathrm{mm}, 4 \mathrm{~mm}, 5 \mathrm{~mm}$, and $1.5 \mathrm{~mm}$ to $0.36 \mathrm{~mm}, 0.34 \mathrm{~mm}, 0.58 \mathrm{~mm}$, and $0.23 \mathrm{~mm}$ respectively $(85.6 \%, 91.5 \%, 88.4 \%$, and $84.7 \%)$ and in the technique of S from $0.54 \mathrm{~mm}$, $0.52 \mathrm{~mm}, 0.5 \mathrm{~mm}$, and $0.2 \mathrm{~mm}$ to $0.09 \mathrm{~mm}, 0.09 \mathrm{~mm}, 0.05 \mathrm{~mm}$, and $0.03 \mathrm{~mm}$ respectively $(83.3 \%, 82.7 \%, 90 \%$, and $85 \%)$. While in the technique of A, the largest decrease was less than $0.2 \mathrm{~mm}(3.1 \%)$ at $0 \mathrm{~dB}$.

\subsubsection{Sinusoidal Boundary Motion with Fixed Scattering Echoes}

\subsubsection{Simulation Model}

Gaussian rf pulse boundary echoes were simulated with sinusoidal boundary motion with total tissue boundary displacement of $1 \mathrm{~mm}$, where $S M_{0}=3.26 \mathrm{~mm}$ and $A_{N}=$ $0.5 \mathrm{~mm}$ in Equation 4.10. Therefore, the boundary depth changes from $2.76 \mathrm{~mm}$ to 3.76 $\mathrm{mm}$ as shown in Figure 4.14. The scattering echoes added to the boundary echo were the same (fixed) for all frames. The simulation model shown in Figure 4.15 assumed that tissue $2\left(\mathrm{TS}_{2}\right)$ is the muscle in relaxation and contraction. Figure 4.16 shows the entire frames of simulated boundary echoes with fixed scattering echoes in M-mode format in $30 \mathrm{~dB}$ SNR. Figure 4.17 shows the simulated signals of the entire frames in A-mode format. In Figure 4.18, it can be said that the sinusoidal boundary motion is more clearly observed with larger SNR values. 


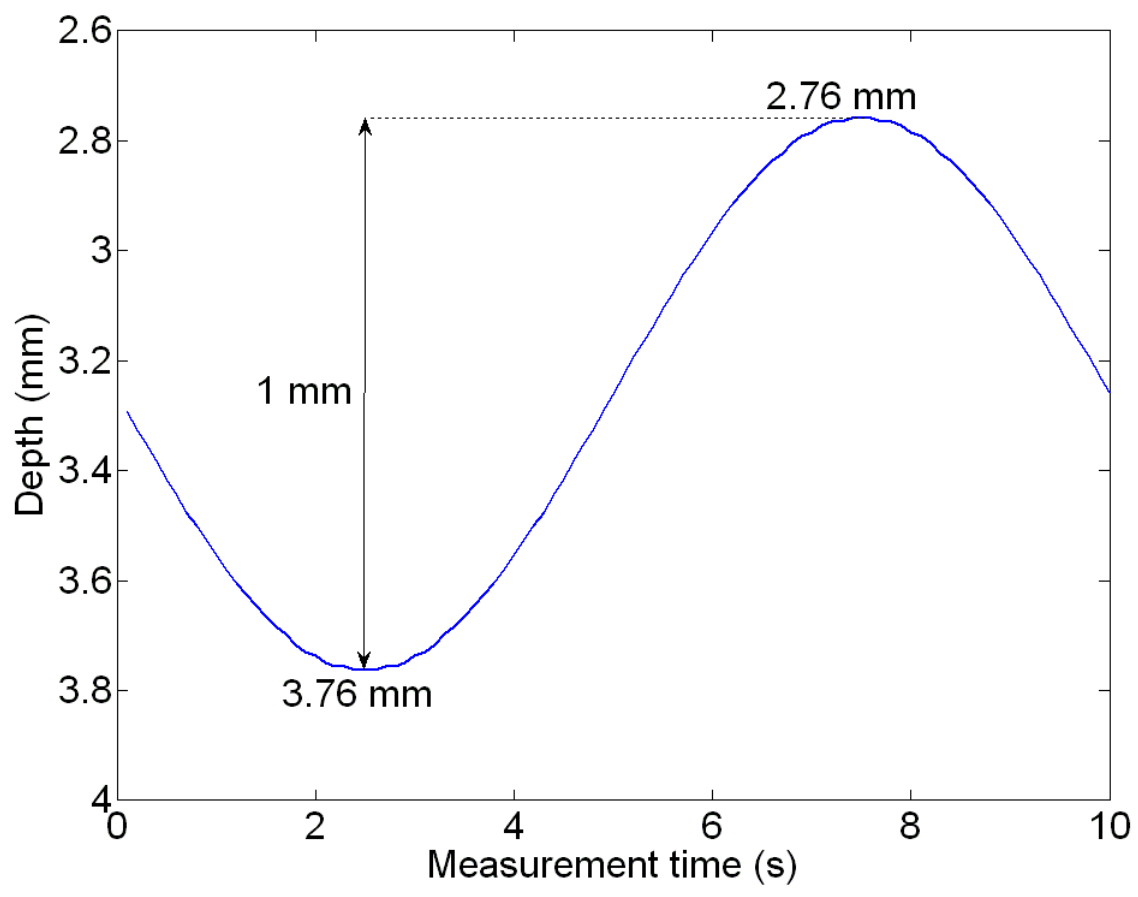

Figure 4.14: The theoretical value (model) motion track of this simulation.

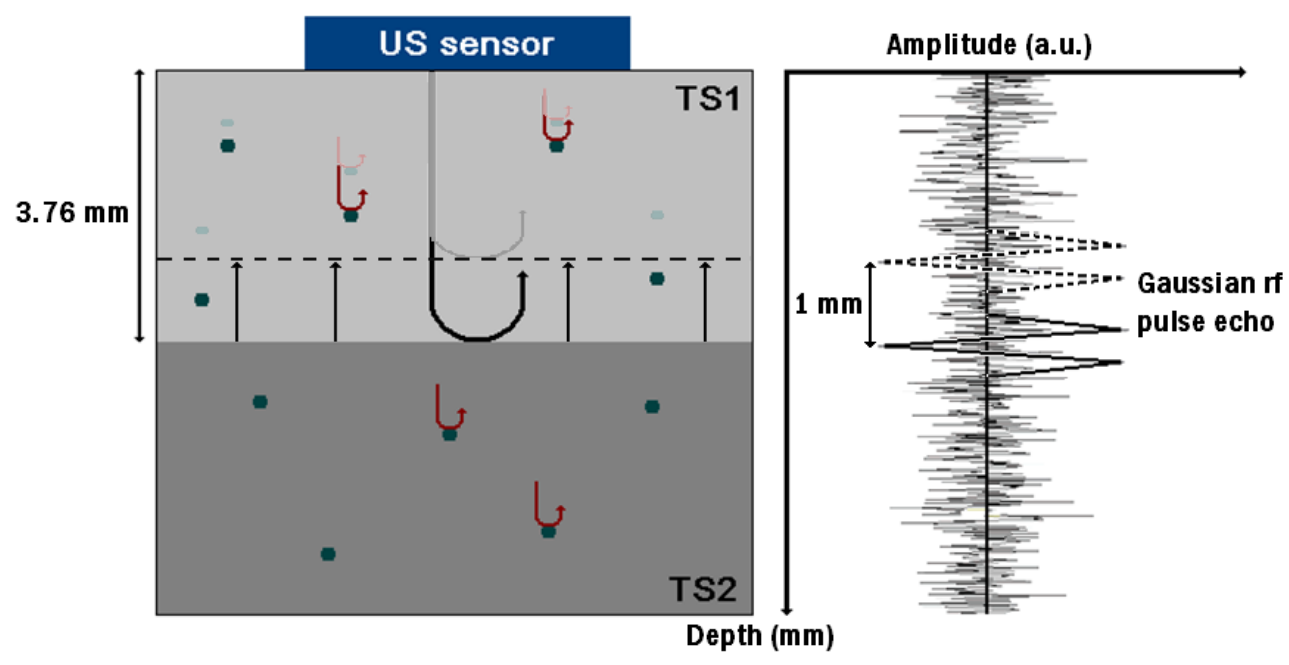

Figure 4.15: The simulation model of the Sinusoidal boundary motion with fixed scattering echoes simulation and the Sinusoidal boundary motion with random scattering echoes. Tissue $2\left(T S_{2}\right)$ is the contracted muscle. (Right figure is confusing. Why there are two boundary echoes? Delete the one at $2.76 \mathrm{~mm}$.) 


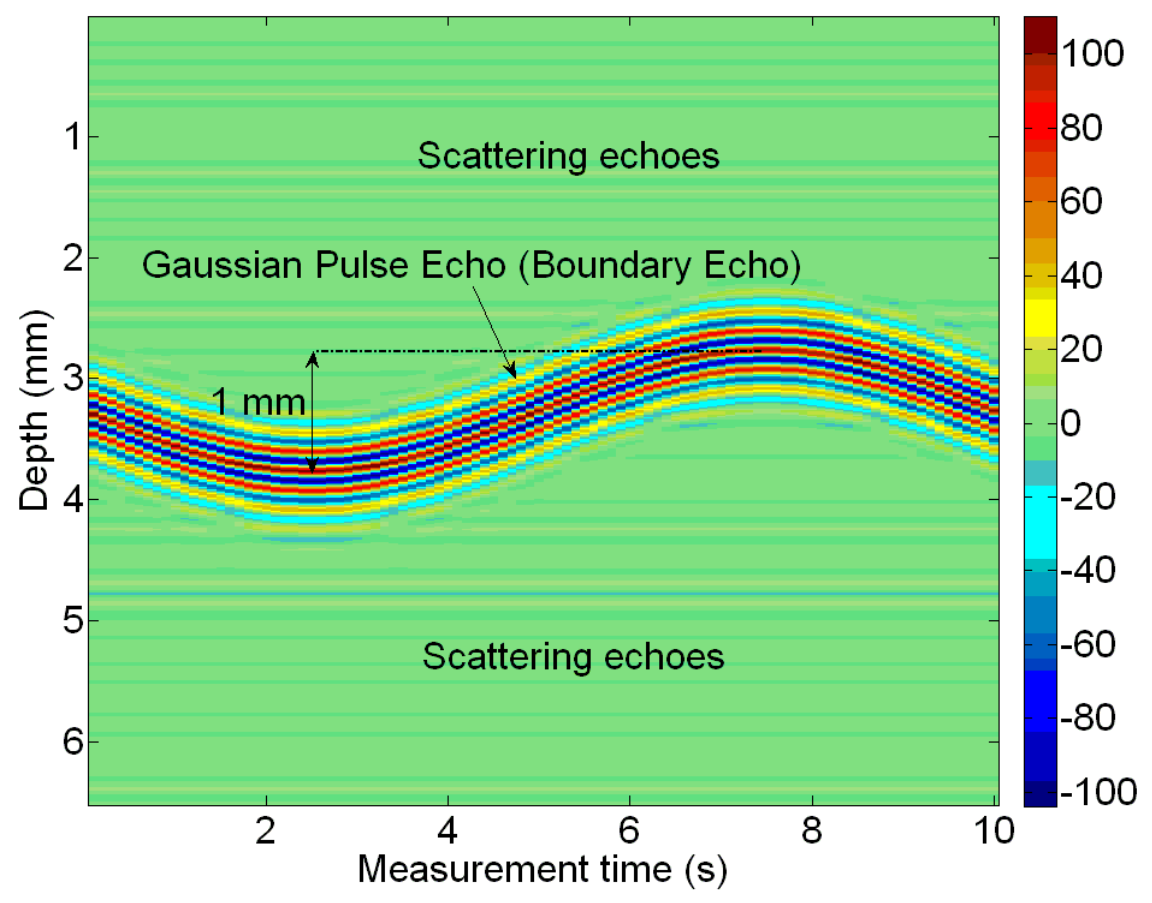

Figure 4.16: : All 100 frames (10 sec at $10 \mathrm{~Hz}$ frame rate) of simulated boundary echoes with sinusoidal boundary motion having fixed scattering echoes with 30 dB SNR in M-mode format. 


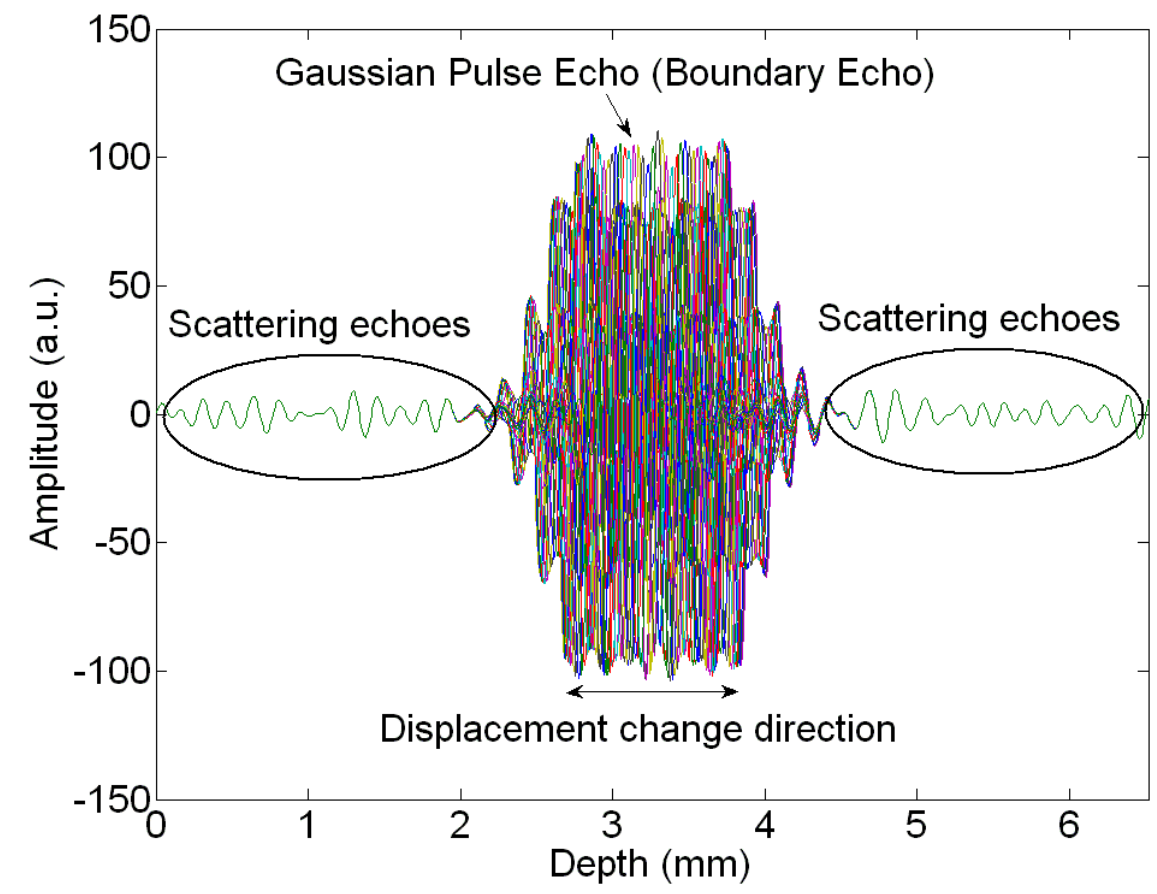

Figure 4.17: All frames of simulated boundary echoes with sinusoidal boundary motion having fixed scattering echoes with $30 \mathrm{~dB}$ SNR in A-mode format. 

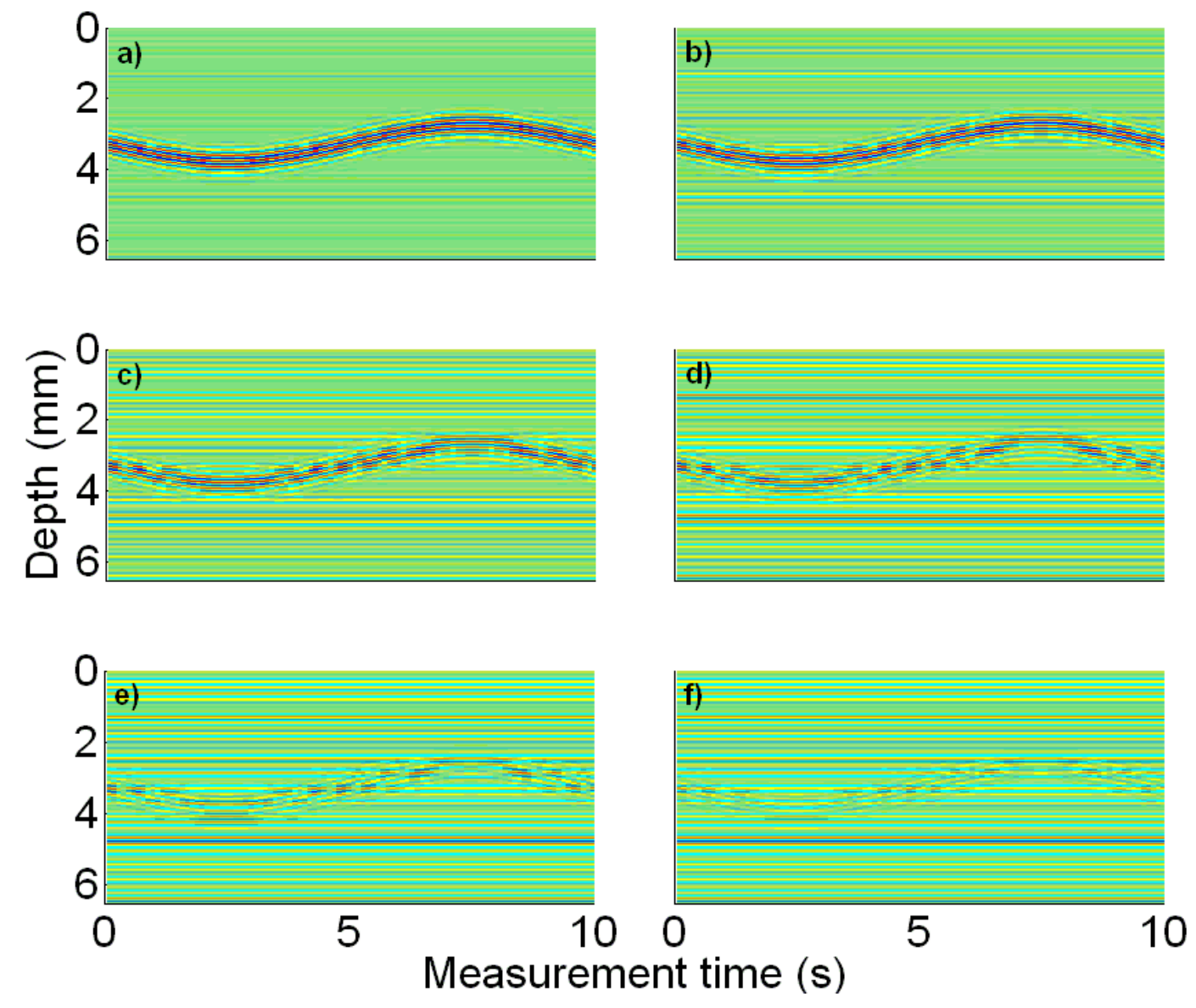

Figure 4.18: Sinusoidal boundary motion with fixed scattering echoes simulation M-mode format (a: $25 \mathrm{~dB}$, b: $20 \mathrm{~dB}$, c: $15 \mathrm{~dB}$, d: $10 \mathrm{~dB}$, e: $5 \mathrm{~dB}$, and f: $0 \mathrm{~dB}$ ).

\subsubsection{Displacement Estimation Results}

Figure 4.19 shows the boundary displacement estimation results obtained by the mentioned techniques in Chapter 3 (a: echo peak detection, b: cross-correlation between the first frame and all frames, c: cross-correlation between two consecutive frames, $d$ : single frequency phase detection, e: mean of narrow band frequencies phase detection and $\mathrm{f}$ : LQ-factorization techniques). The center frequency for the techniques of $\mathrm{d}$, e, and $\mathrm{f}$ 
and the bandwidth frequencies for the technique e were chosen to be the same values as in the previous simulation (Section 4.2.1.2).

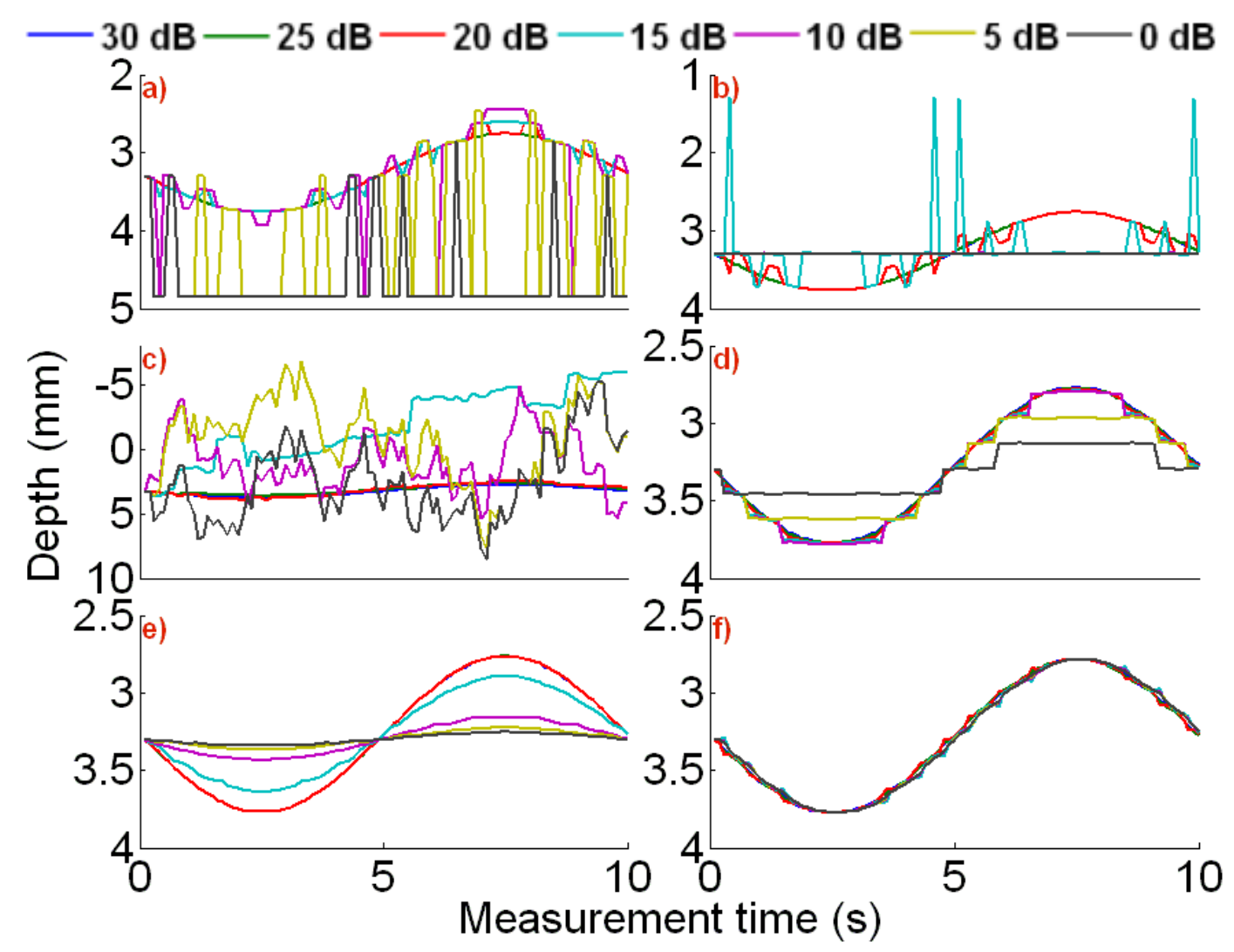

Figure 4.19: Boundary track of each technique in seven different SNR values of $30,25,20,15,10,5$, and $0 \mathrm{~dB}$ (a: echo peak detection, b: cross-correlation between firs frame and all frames, c: cross-correlation between two consecutive frames, d: single frequency phase detection, e: mean of narrow band frequencies phase detection, and f: LQ-factorization).

As seen in Figure 4.19, the techniqus (a) and (b) estimated a fixed displacement at the depth of $4.80 \mathrm{~mm}$ and $3.35 \mathrm{~mm}$ respectively at $10 \mathrm{~dB}$ and lower SNR. These estimation error of fixed displacements were caused by a larger amplitude of scattering echoes than those of the boundary echo since the scattering echoes were fixed. A large scattering echo can be seen at $4.80 \mathrm{~mm}$ in Figures 4.16, 4.17 and 4.18. Another large scattering echoe at $3.35 \mathrm{~mm}$ is not visilbe in these figures because of the overrapping 
with the boundary echoes. The phase detection techniques of (d) and (e) in Figure 4.19 tracked the sinusoidal motion well but the accuracy had reduced in lower SNR values. The single frquency phase detection technique (d) estimated the total displacement of $0.30 \mathrm{~mm}$ and $0.60 \mathrm{~mm}$ in $0 \mathrm{~dB}$ and $5 \mathrm{~dB}$ SNR respectively. While the mean of narrow band frequencies phase detection technique (e) estimated the total diplacement of 0.05 $\mathrm{mm}, 0.10 \mathrm{~mm}, 0.20 \mathrm{~mm}$, and $0.70 \mathrm{~mm}$ in $0 \mathrm{~dB}, 5 \mathrm{~dB}, 10 \mathrm{~dB}$, and $15 \mathrm{~dB}$ SNR respectively. These estimated total thickness changes was less than the theoretical value of $1 \mathrm{~mm}$. This is probably because both the simulated boundary echoes and the scattering echoes had the same center frequency and bandwidth of $5 \mathrm{MHz}$ and $0.025 \mathrm{MHz}^{2}$ respectively. With lower SNR values, the magnitudes of frequency spectrum of the boundary echo and the scattering echoes became comparable. Moreover, one or more of these frequencies could be noise. Hence, the noise reduces the averaging? effectiveness to improve the accuracy.

The LQ-factorization in Figure 4.19f successfully tracked the motion with better accuracy even in lower SNR values. The results show unsmooth tracknig in lower SNR values, but still had the best accuracy among all other techniques.

The absolute value of the simulated echoes was also used for the simulate experiments of boundary displacement estimation. The comparison between the raw signals and the absolute value signals is presented in Figure 4.20. Results are discussed in the next section. 


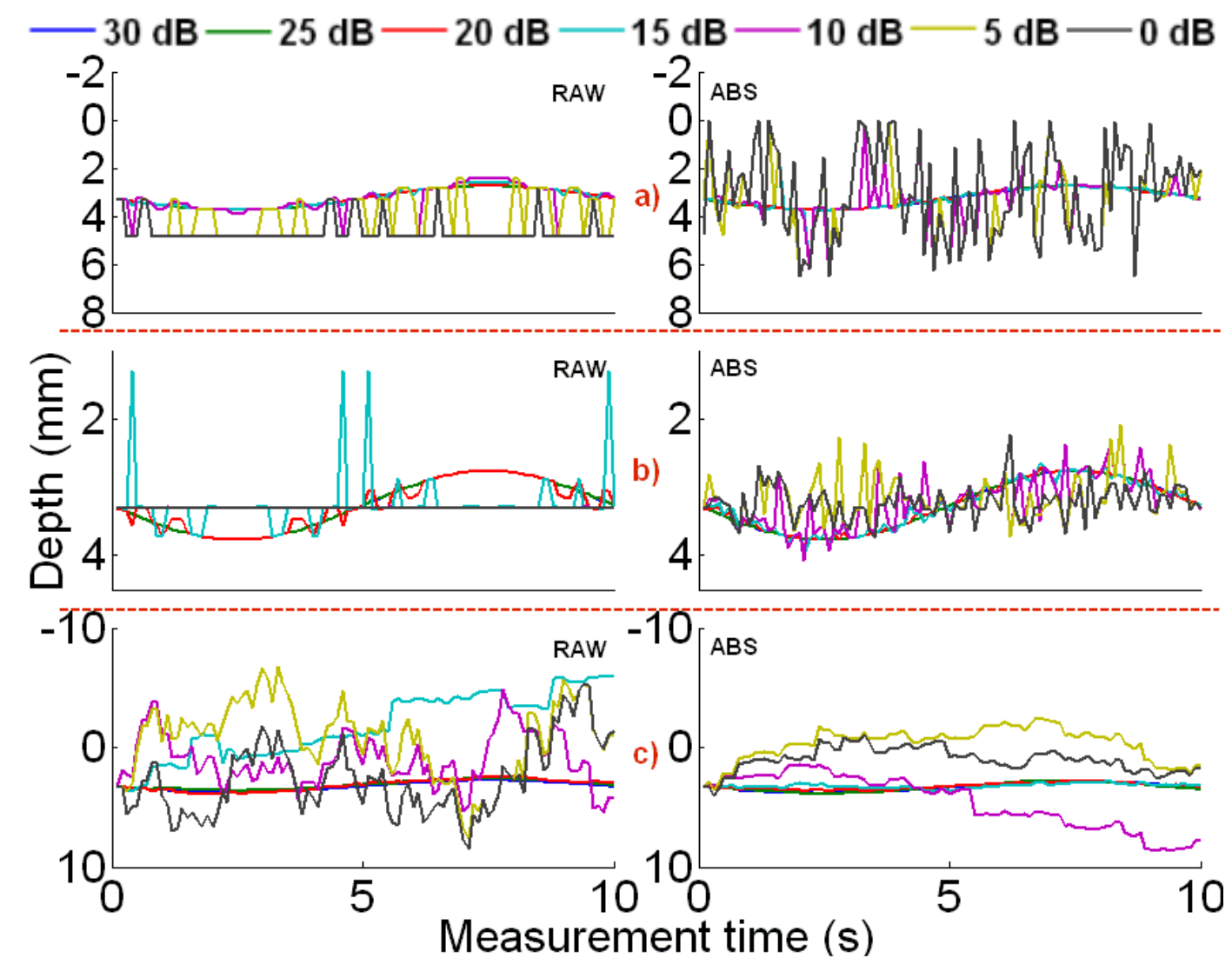

Figure 4.20: The comparisons between applying the techniques on raw signals and on signals absolute value for boundary depth estimation for simulation 2 where a) echo peak detection, b) cross-correlation between first frame and all frames, and c) cross-correlation between two consecutive frames.

\subsubsection{Root Mean Squared Error (RMSE)}

As discussed in Section 4.2.1.3, the RMSE values were obtained from the results shown in Figures 4.19 and 4.20 to compare the techniques with respect to the SNR values. The results are presented in Figures 4.21 and 4.22. 


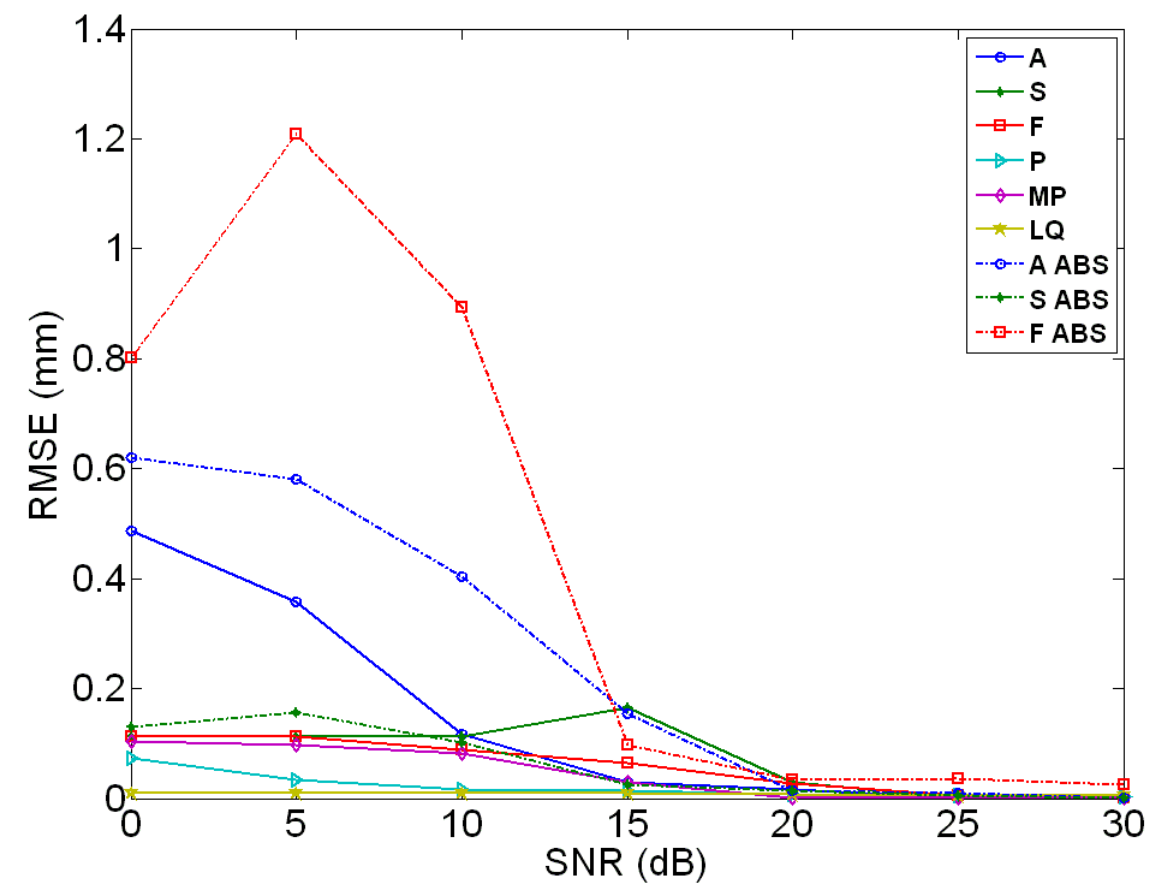

Figure 4.21: RMSE values of all techniques including the absolute value analysis for $A, S$ and $F$ in 30, 25, 20, 15, 10, 5, and $0 \mathrm{~dB}$ SNR (A: echo peak detection, $S$ : cross-correlation between first frame and all frames, $\mathrm{F}$ : cross-correlation between two consecutive frames, P: single frequency phase detection, MP: mean of narrow band frequencies phase detection, LQ: LQ-factorization, and ABS: absolute value).

As shown in Figure 4.21, using the absolute value of the signals with the techniques of $\mathrm{A}, \mathrm{S}$, and $\mathrm{F}$ (A $\mathrm{ABS}, \mathrm{S} \mathrm{ABS}$, and $\mathrm{F}$ ABS) did not improve the results accuracy, unlike the previous simulation results presented in Section 4.2.1.3. Moreover, these three techniques in time domain signal analysis had less accuracy compared with the frequency domain techniques of P: single frequency phase detection, MP: mean of narrow band frequencies phase detection, and LQ: LQ-factorization.

From Figure 4.22, mean of narrow band frequencies phase detection (MP) had the best accuracy among all techniques in 30-20 dB SNR (less than $0.003 \mathrm{~mm}$ RMSE). However, the RMSE increased in $15 \mathrm{~dB}$ and lower SNR values. The LQ-factorization 
technique had less accuracy in 30-25 dB than both phase detection techniques, but its accuracy was still very good (0.01 mm RMSE) The LQ-factorization technique had constant accuracy even in low SNR ranges.

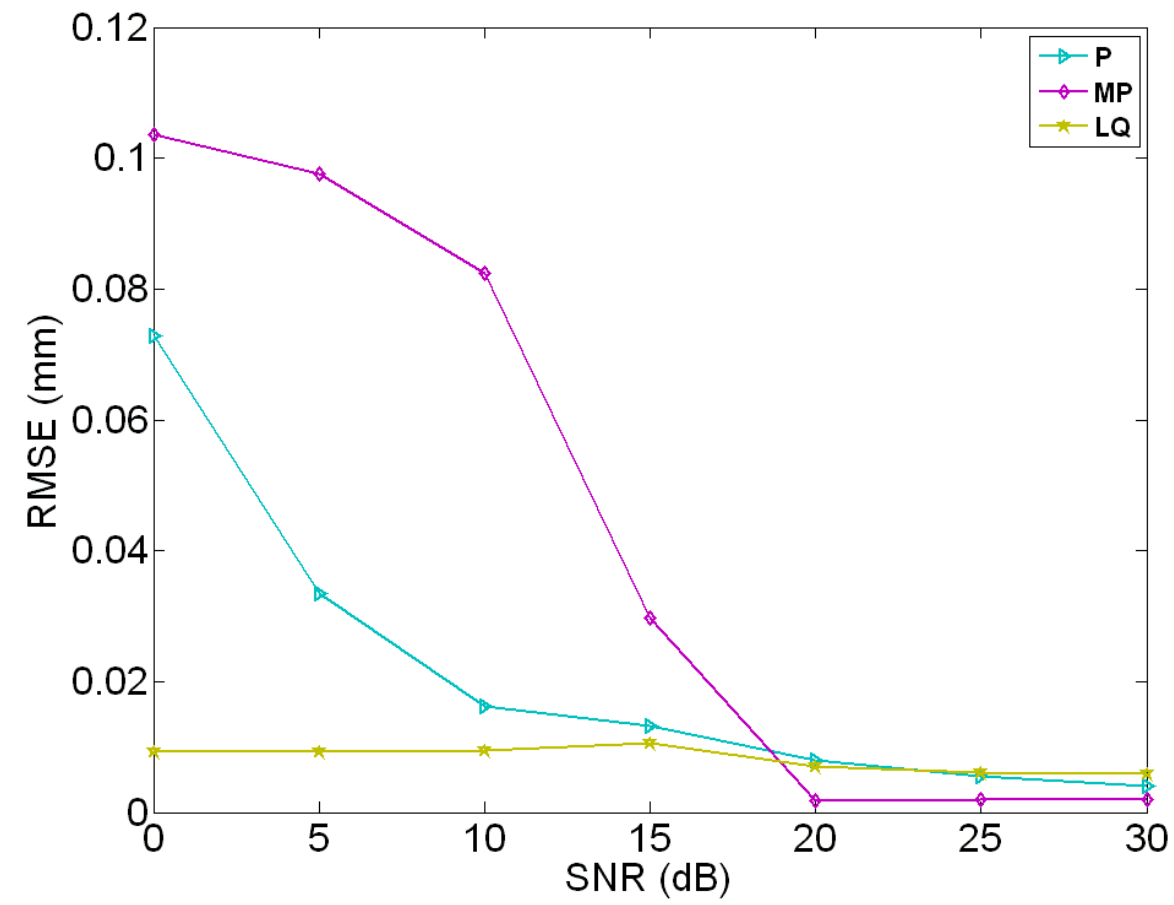

Figure 4.22: RMSE values of only single frequency phase detection (P), mean of narrow band phase detection (MP), and LQ factorization (LQ).

\subsubsection{Sinusoidal Boundary Motion with Random Scattering Echoes}

\subsubsection{Simulation Model}

Section 4.2.1 has examined the random scattering echoes with no motion boundary echo. While in Section 4.2.2, the scattering echoes were fixed and examined with sinusoidal boundary motion. In this section, the random scattering echoes are 
examined along with the sinusoidal boundary motion with the same total displacement of $1 \mathrm{~mm}$ where $A_{N}=0.5 \mathrm{~mm}$ and $S M_{0}=3.26 \mathrm{~mm}$ as it can be seen in Figure 4.14. This simulation is the closest model to the in-vivo experiment. Figure 4.15 is the model of this simulation. Figure 4.23 shows the simulated boundary echoes with the random scattering echoes in M-mode format in $30 \mathrm{~dB}$ SNR.

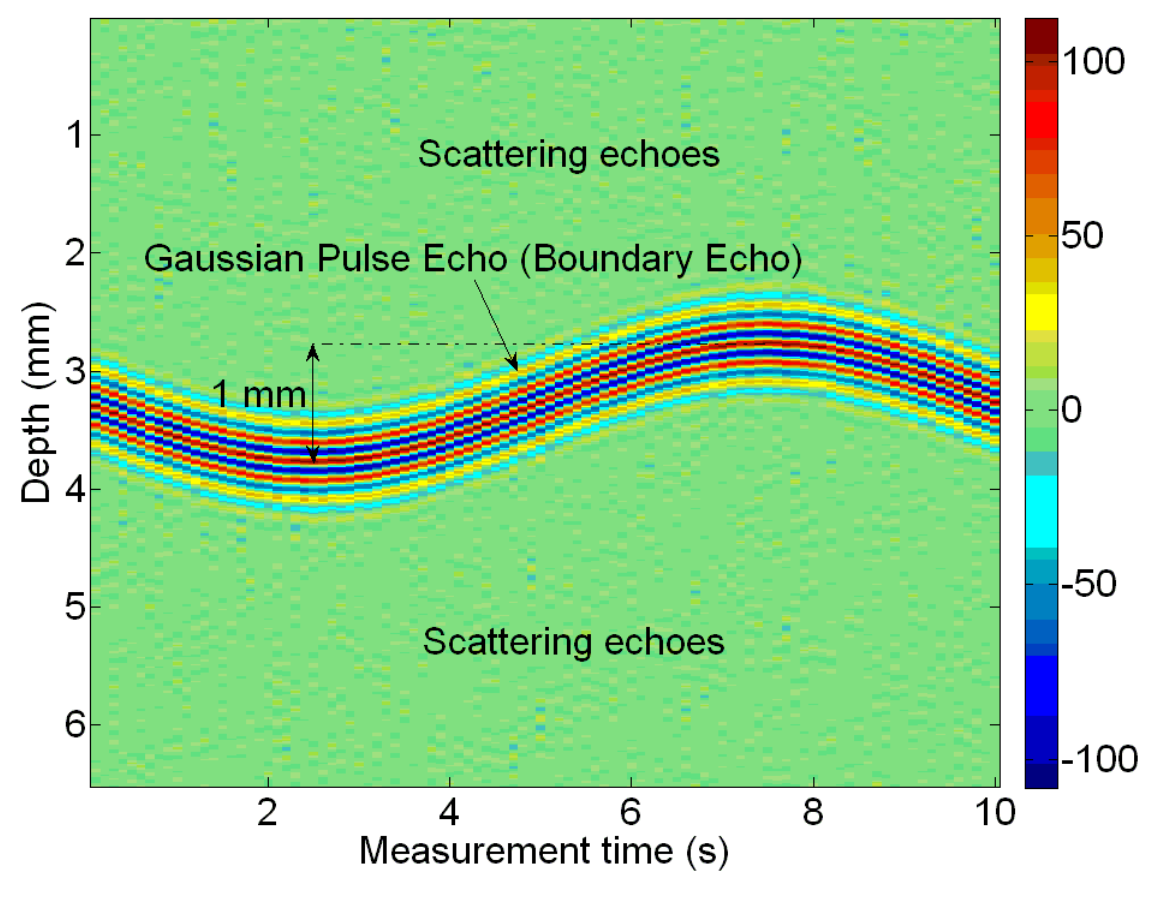

Figure 4.23: All 100 frames (10 sec at $10 \mathrm{~Hz}$ frame rate) of simulated boundary echoes with sinusoidal boundary motion having random scattering echoes with 30 dB SNR in M-mode format. 


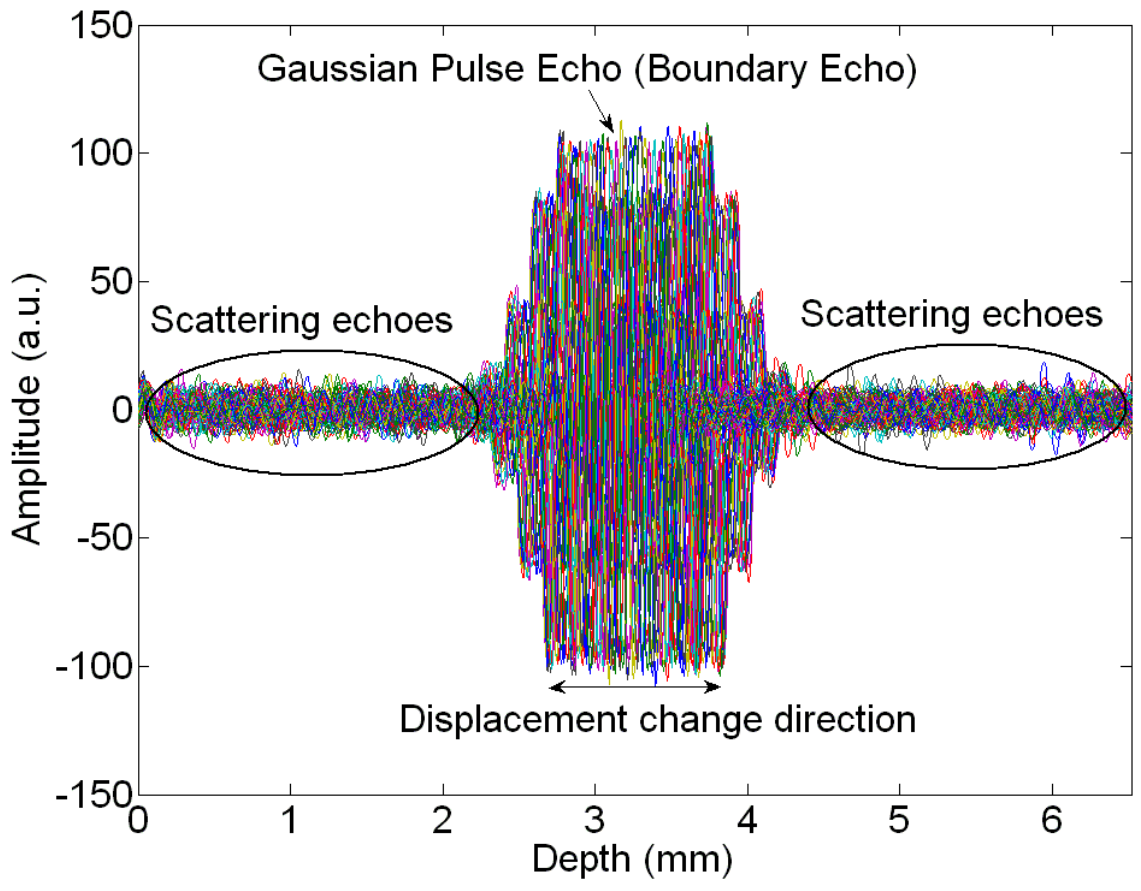

Figure 4.24: All frames of simulated boundary echoes with sinusoidal boundary motion having random scattering echoes with $30 \mathrm{~dB}$ SNR in A-mode format. 

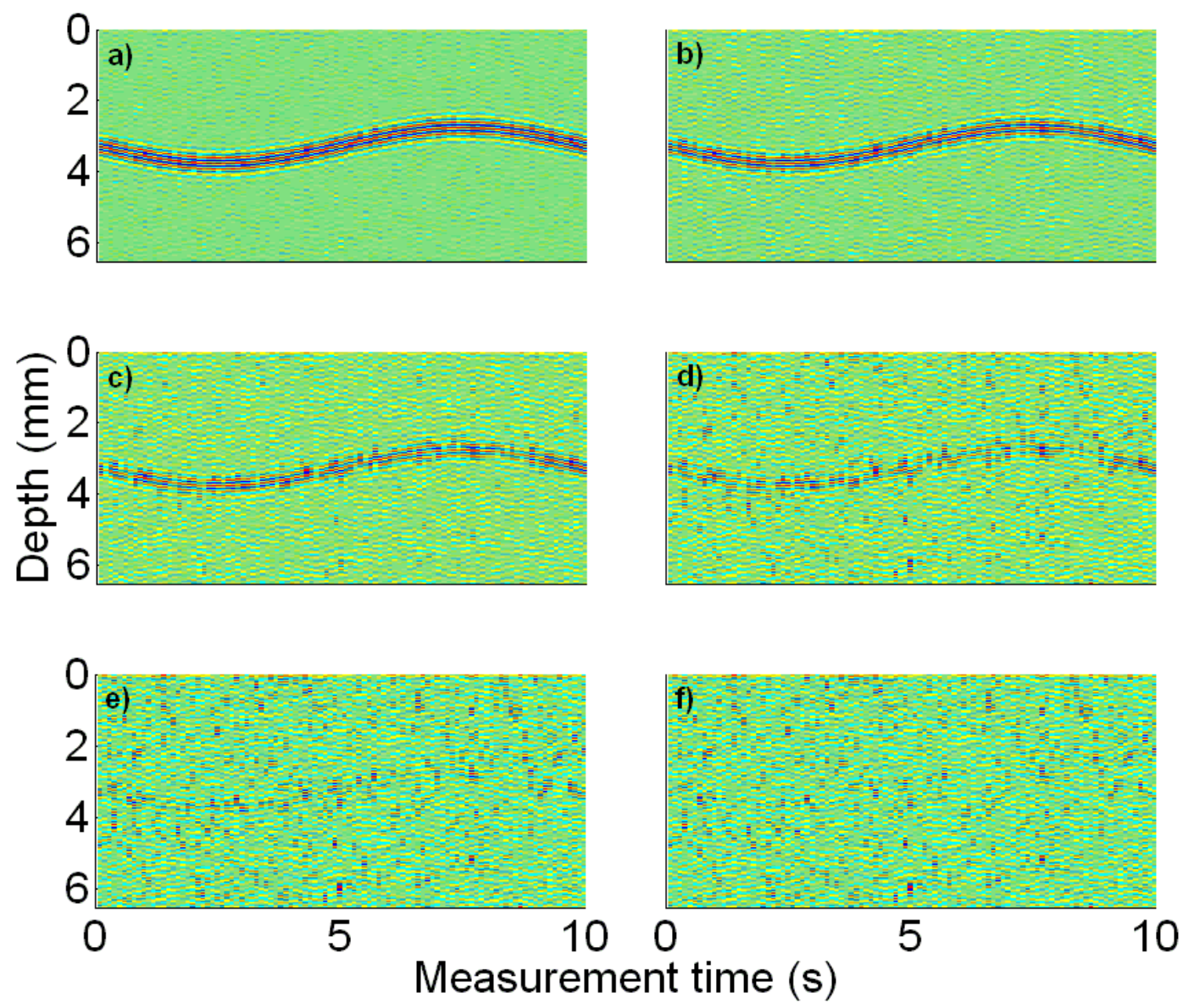

Figure 4.25: Sinusoidal boundary motion with random scattering echoes simulation M-mode format (a: $25 \mathrm{~dB}$, b: $20 \mathrm{~dB}$, c: $15 \mathrm{~dB}, \mathrm{~d}: 10 \mathrm{~dB}$, e: $5 \mathrm{~dB}$, and f: ( dB).

The sinusoidal boundary motion is more clearly observed with larger SNR values in Figure 4.25 until it is no longer can be seen in Figure 4.25e and $\mathrm{f}$.

\subsubsection{Displacement Estimation Results}

Similar processes to the previous simulations have done here. Figure 4.26 shows the boundary displacement estimation results obtained by the six techniques a: echo peak detection, b: cross-correlation between the first frame and all frames, c: cross-correlation 
between two consecutive frames, d: single frequency phase detection, e: mean of narrow band frequencies phase detection and f: LQ-factorization techniques.
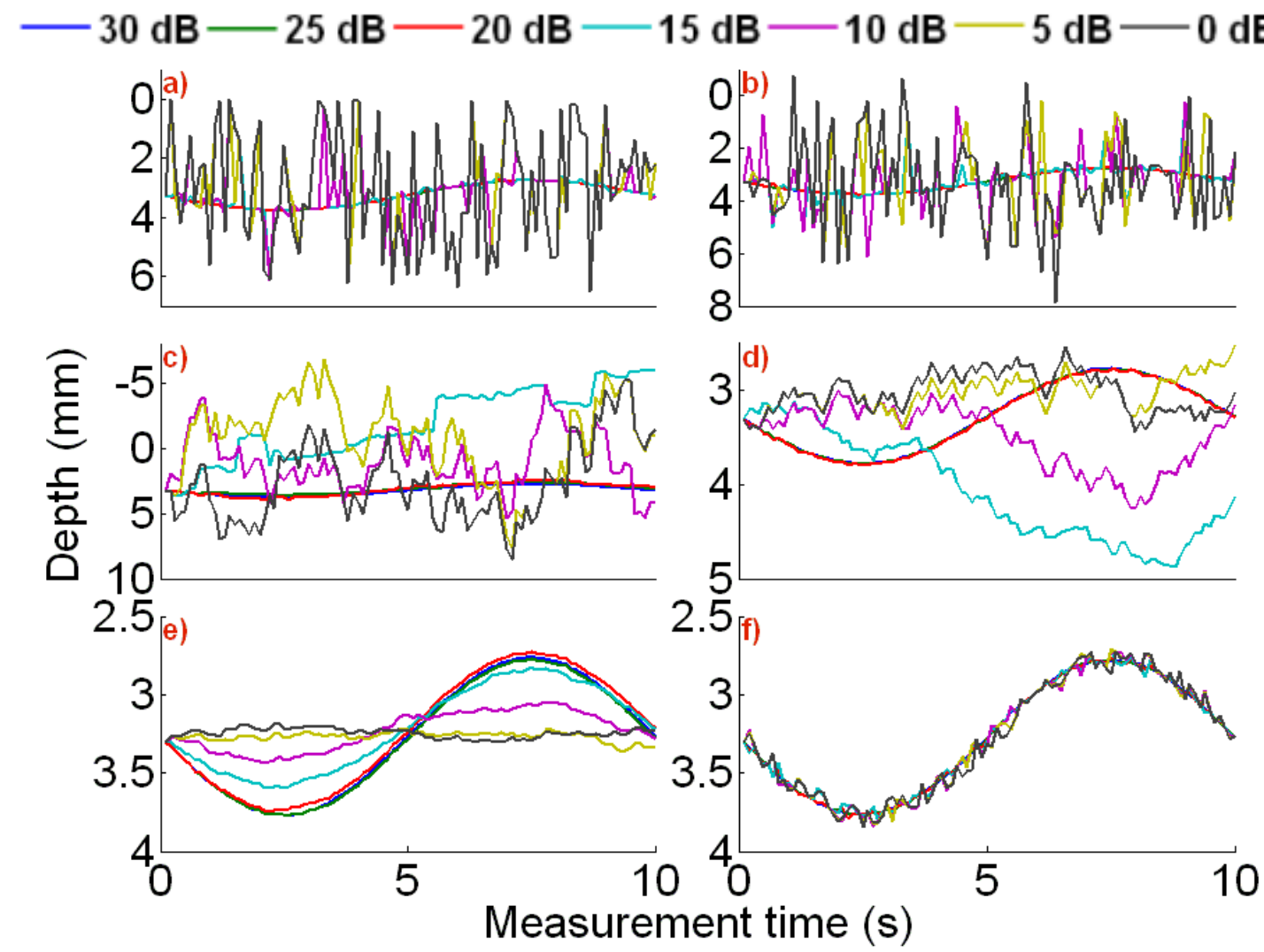

Figure 4.26: Boundary track of each technique in seven different SNR values of $30,25,20,15,10,5$, and $0 \mathrm{~dB}$ (a: echo peak detection, b: cross-correlation between firs frame and all frames, c: cross-correlation between two consecutive frames, $d$ : single frequency phase detection, e: mean of narrow band frequencies phase detection, and f: LQ-factorization).

As can be seen in the above figure, the techniques (a) and (b) estimate the sinusoidal boundary motion at $15 \mathrm{~dB}$ and higher SNR. However, the displacement estimation has become noisy at $10 \mathrm{~dB}$ and lower SNR. The estimation error of the noisy displacements were caused by a larger amplitude of scattering echoes than those of the boundary echo since the scattering echoes were random distributed in the frames. In (c), the estimation error is very large at $15 \mathrm{~dB}$ and lower SNR value. The reason behind the 
very large error was the changing of the first frame in every cross-correlation process. Hence, the target of the correlation was changed. The single frequency phase detection in the previous simulation in Section 4.2.2 has estimated the sinusoidal displacement well at all the SNR value in Figure 4.19d but with lower accuracy in lower SNR values. However, in this simulation, the displacement estimation had large errors at $15 \mathrm{~dB}$ SNR and below as seen in Figure 4.26d. Therefore, it can be concluded that the random distribution of the scattering echoes have reduced the effectiveness of the single frequency phase detection technique. The technique (e) in the above figure tracked the sinusoidal motion well but the accuracy reduces in lower SNR values. The random scattering echoes have no significant impact on this technique, unlike with the single frequency phase detection technique. Therefore, the mean of narrow band frequencies phase detection has overcame with the random scattering echoes limitation.

The LQ-factorization in Figure 4.26f successfully tracked the sinusoidal motion with good accuracy at all SNR values. The displacement estimation had become unsmooth with lower SNR. However, LQ-factorization can still be estimates the overall motion with a total of $1 \mathrm{~mm}$ boundary displacement at all SNR values.

The absolute value of the simulated echoes was also used for this simulation experiment as done with the previous simulations. The comparison between the raw signals and the absolute value signals is presented in Figure 4.27. Results are discussed in the next section. 


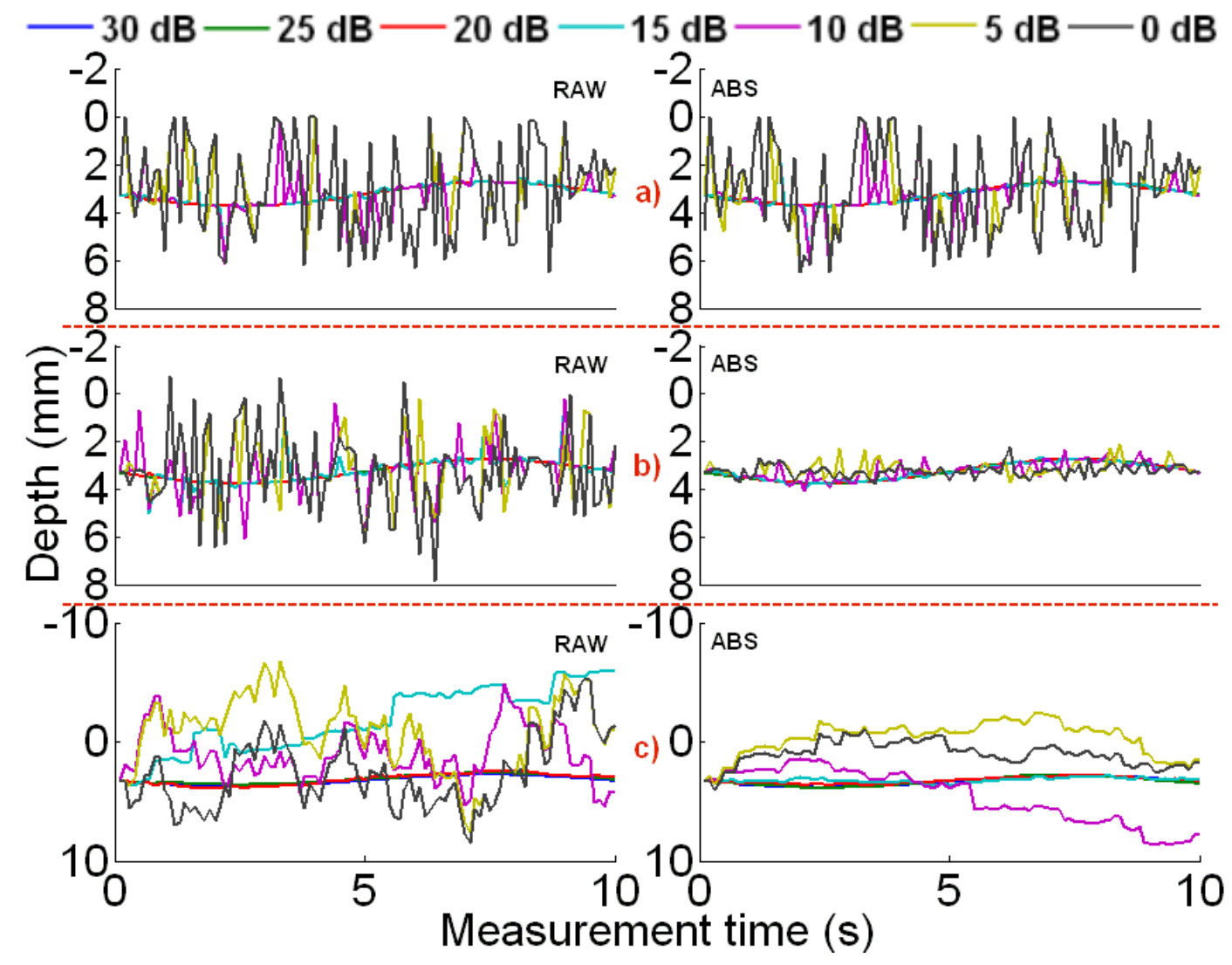

Figure 4.27: The comparisons between applying the techniques on raw signals and on signals absolute value for boundary depth estimation for simulation 3 where a) echo peak detection, b) cross-correlation between first frame and all frames, and c) cross-correlation between two consecutive frames.

\subsubsection{Root Mean Squared Error (RMSE)}

The RMSE values as it can be seen in Figures 4.28 and 4.29 were obtained from the results shown in Figures 4.26 and 4.27. 


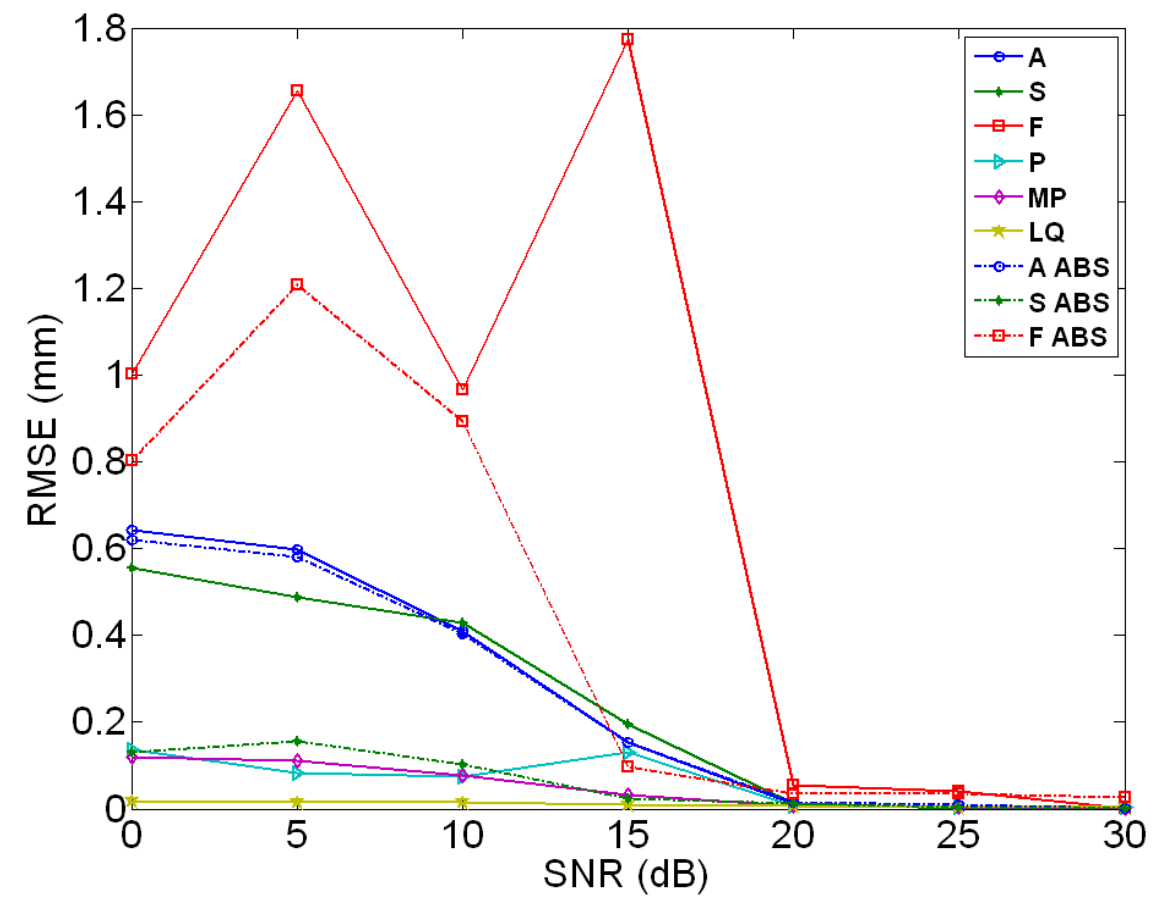

Figure 4.28: RMSE values of all techniques and the absolute value analysis in 30, 25, 20, 15, 10, 5, and 0 dB SNR (A: echo peak detection, S: cross-correlation between first frame and all frames, $\mathrm{F}$ : cross-correlation between two consecutive frames, P: single frequency phase detection, MP: mean of narrow band frequencies phase detection, LQ: LQ-factorization, and ABS: absolute value).

As shown in Figure 4.28, signal absolute value improved the results of A: echo peak detection, S: cross-correlation between first frame and all frames, and F: crosscorrelation between two consecutive frames. It can be observed that in both crosscorrelation techniques (S) and (F), the improvemets were large and the RMSE decreased from $1 \mathrm{~mm}, 1.7 \mathrm{~mm}, 1 \mathrm{~mm}$, and $1.8 \mathrm{~mm}$ to $0.8 \mathrm{~mm}, 1.2 \mathrm{~mm}, 0.9 \mathrm{~mm}$, and $0.15 \mathrm{~mm} \mathrm{(20}$ $\%, 29.4 \%, 10 \%$, and $91.7 \%$ ) at $0 \mathrm{~dB}, 5 \mathrm{~dB}, 10 \mathrm{~dB}$, and $15 \mathrm{~dB}$ SNR respectively for the cross-correlation between two consecutive frames and from $0.55 \mathrm{~mm}, 0.5 \mathrm{~mm}, 0.4 \mathrm{~mm}$, and $0.2 \mathrm{~mm}$ to $0.13 \mathrm{~mm}, 0.16 \mathrm{~mm}, 0.1 \mathrm{~mm}$, and $0.05 \mathrm{~mm}$ respectively $(76.3 \%, 68 \%$, $0.75 \%$, and $75 \%$ ) for the cross-correlation between first frame and all frames. The 
largest decrease of RMSE in A: echo peak detection was less than $0.01 \mathrm{~mm}(1.6 \%)$. Even with the improvement of (A), (S), and (F), the techniques of P: single frequency phase detection, MP: mean of narrow band frequencies phase detection, and LQ: LQfactorization had better accuracy exept for the (S ABS) compared with (P). From Figure 4.29, the (MP) accuracy was the best in $30 \mathrm{~dB}$ but with a small differeance than the (P) and (LQ) with less than $0.0025 \mathrm{~mm}$ RMSE. However, (P) and (MP) RMSE increased in $20 \mathrm{~dB}$ and lower SNR values, while the (LQ) had constant accuracy entire SNR values.

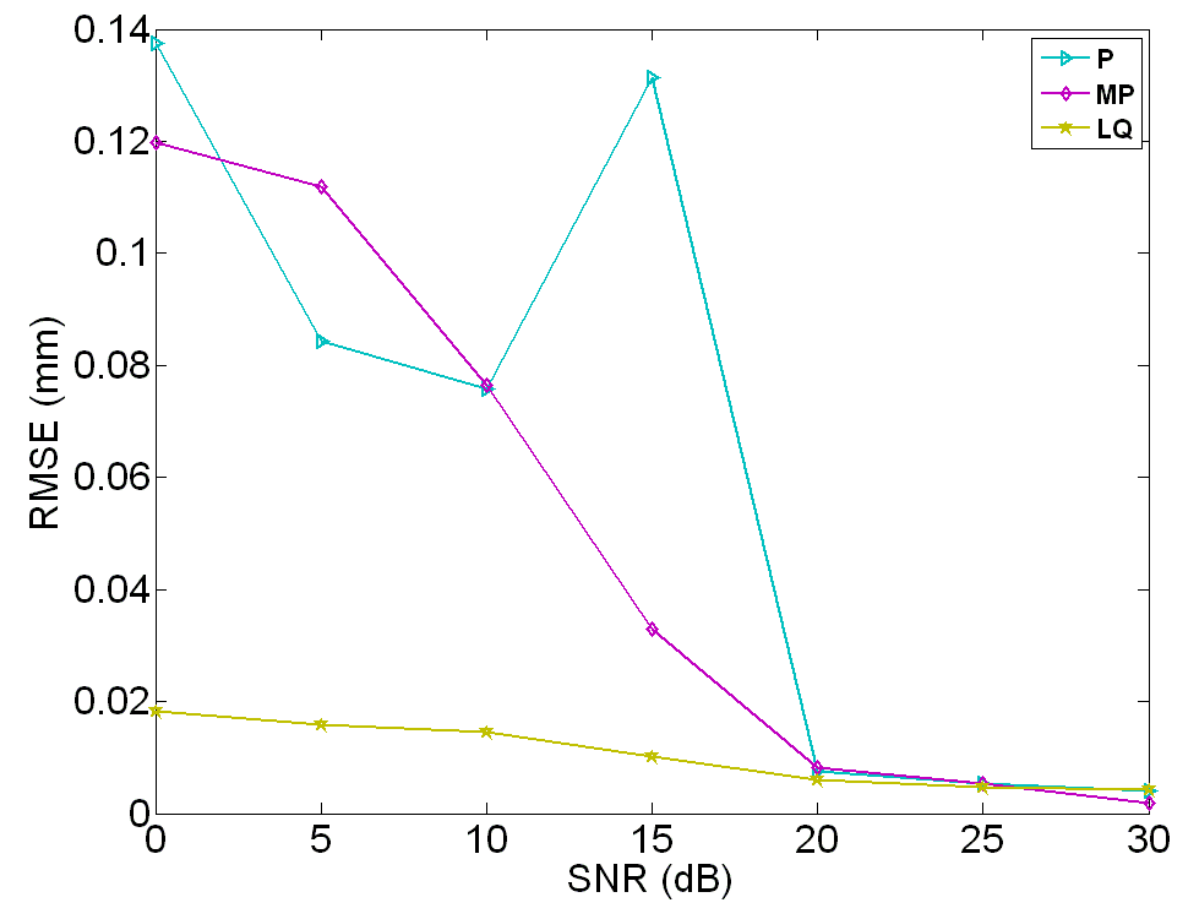

Figure 4.29: RMSE values of only single frequency phase detection $(\mathrm{P})$, mean of narrow band phase detection (MP), and LQ factorization (LQ). 


\section{Chapter 5}

\section{In-vivo Experiment}

In Chapter 4, the effects of the random scattering echoes on tracking tissue boundary motion were examined by means of numerical simulation experiments. In this chapter, in-vivo experiments at the forearm are conducted using a wearable ultrasonic sensor. The acquired ultrasonic signals are analysed using the signal processing techniques mentioned in Chapter 3. The results of tracking the boundary displacement and tissue thickness changes due to muscle contraction are presented and discussed as well.

\subsection{Experimental model}

The study was performed in compliance with the Carleton University Research Ethics Board and with the approval of the board. In the experiment, a wearable ultrasonic sensor (see Section 5.4.1) was placed on a right proximal forearm of a human subject (Male 28 years old). Ultrasonic signals were acquired while isometric muscle contraction was performed. Four tissue boundary echoes were detected between the ultrasonic sensor and the bone. Figure 5.1 shows the experimental model of this study. Through human anatomy study of the forearm in Section 5.3, these four tissues might be as follow: $T S_{1}$ (fat), $T S_{2}$ (muscle), $T S_{3}$ (nerve or muscle), and $T S_{4}$ (muscle). 


\subsection{Tissue Thickness Measurement}

During isometric muscle contraction, muscle thickness changes. In order to investigate the thickness variations, ultrasonic signals were acquired at a proximal right forearm in the in-vivo experiment. The signals were analysed to determine the tissue thickness using the discussed techniques in Chapter 3 and 4. Echo depth at each frame can be measure by Equation 3.1. In this research, we assumed that the propagation velocities are equal in all soft tissues. The fixed velocity $\left(v_{i}=v\right)$ was assumed to be $1630 \mathrm{~m} / \mathrm{s}$ which is the velocity in muscle from [104, 105]. Hence, Equation 3.1 can be modified to:

$$
d_{i, j}=\frac{v \cdot T_{i, j}}{2}
$$

Thickness of each tissue layer $T H_{i, j}$ is obtained by Equation 3.3 or by:

$$
T H_{i, j}=\frac{v \cdot \Delta T d_{i, j}}{2}
$$

Total thickness $\mathrm{TH}_{\text {total }_{j}}$ between the ultrasonic sensor and the bone at the $j^{\text {th }}$ frame can be obtained by:

$$
T H_{\text {total }, j}=\sum_{i=1}^{M} T H_{i, j}
$$


where $M$ is the total number of detected boundary echoes between the ultrasonic sensor and the bone.

The total thickness $\left(\mathrm{TH}_{\text {total }_{j}}\right)$ is also equivalent to the depth of boundary located between a tissue and the bone (the fourth tissue in this study).

$$
T H_{\text {total }, j}=d_{4, j}
$$

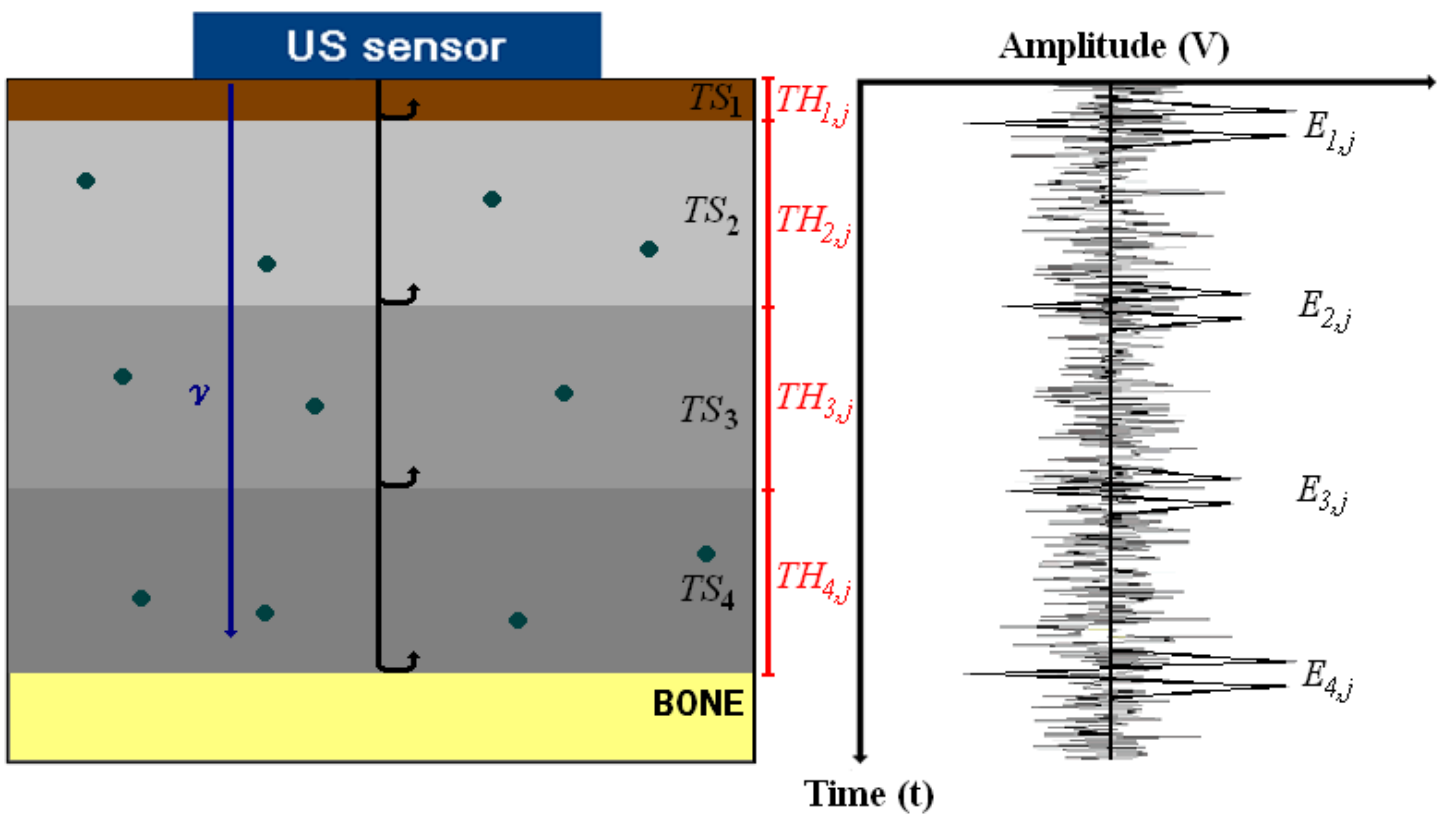

Figure 5.1: Ultrasonic pulse-echo measurement model of in-vivo experiment for tracking tissue boundaries.

\subsection{Forearm Anatomy and US Sensor Placement:}

Forearm representation has two different positions: supination and pronation. Supination is a position of the forearm when the palm is facing up while the human is lying on the back. Pronation is the opposite (palm is facing downward). In the supination 
position, both bones of radius and ulna are parallel. In pronation, the radius crosses the ulna interiorly causing the hand to rotate (Figure 5.2) [106-109]. Therefore, forearm structure is not the same in both positions of a particular area. There are muscles responsible for the rotation of the forearm while other muscles do not intervene in that action such as pronator teres, pronator quadrates, brachioradialis, supinator, and anconeus. Furthermore, specific muscles appear in the posterior side of the forearm. Understanding the forearm anatomy helps us to identify the tissues beneath the ultrasonic sensor attached.

Figure 5.3 presents a posterior view of right forearm muscles. In the posterior side of the forearm, extensor muscles are located in the posterior side of the forearm. While, flexor muscles locate in the opposite side of the forearm (anterior side). Hence, the first tissue layer after the fat beneath the ultrasonic sensor might be an extensor muscle. Table 5.1 shows the kinds of muscles that appear on each side of the forearm (posterior and anterior) and their level of depth [106, 109]. 
Rotators of Radius - Supination

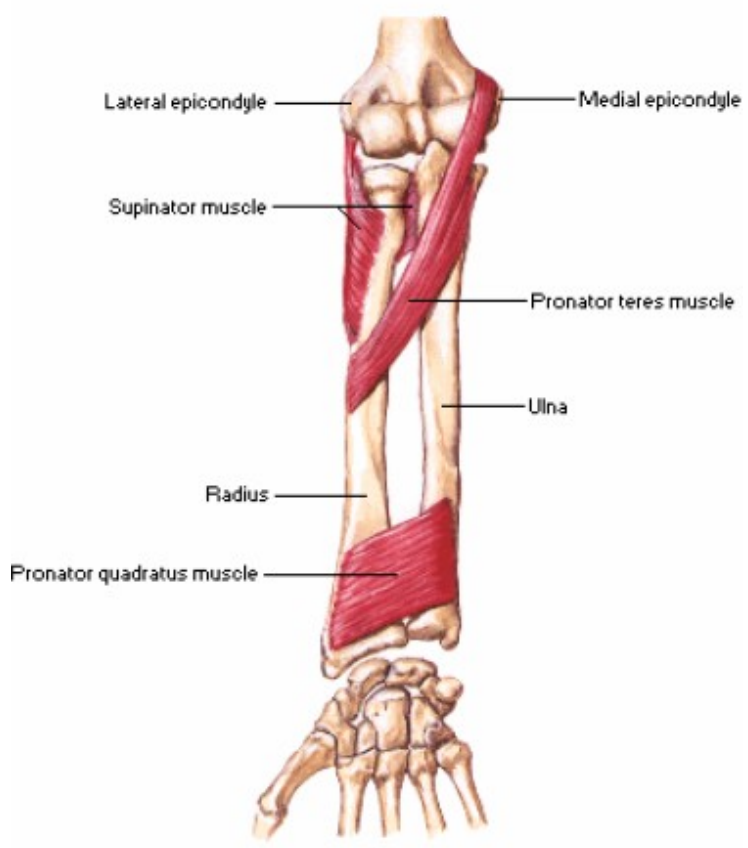

Rotators of Radius - Pronation

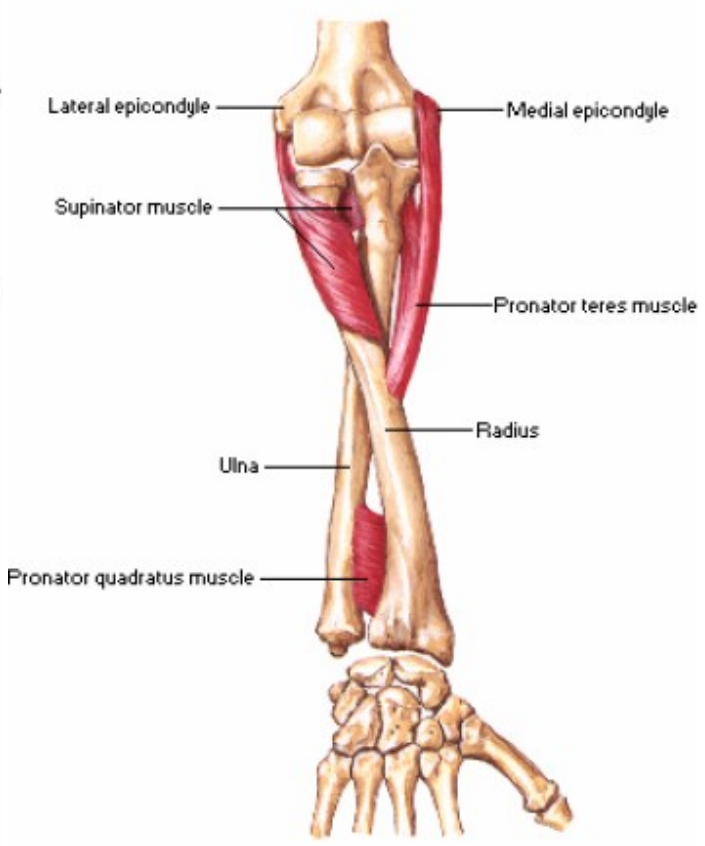

Figure 5.2: Supination and pronation position of right forearm [107]. 


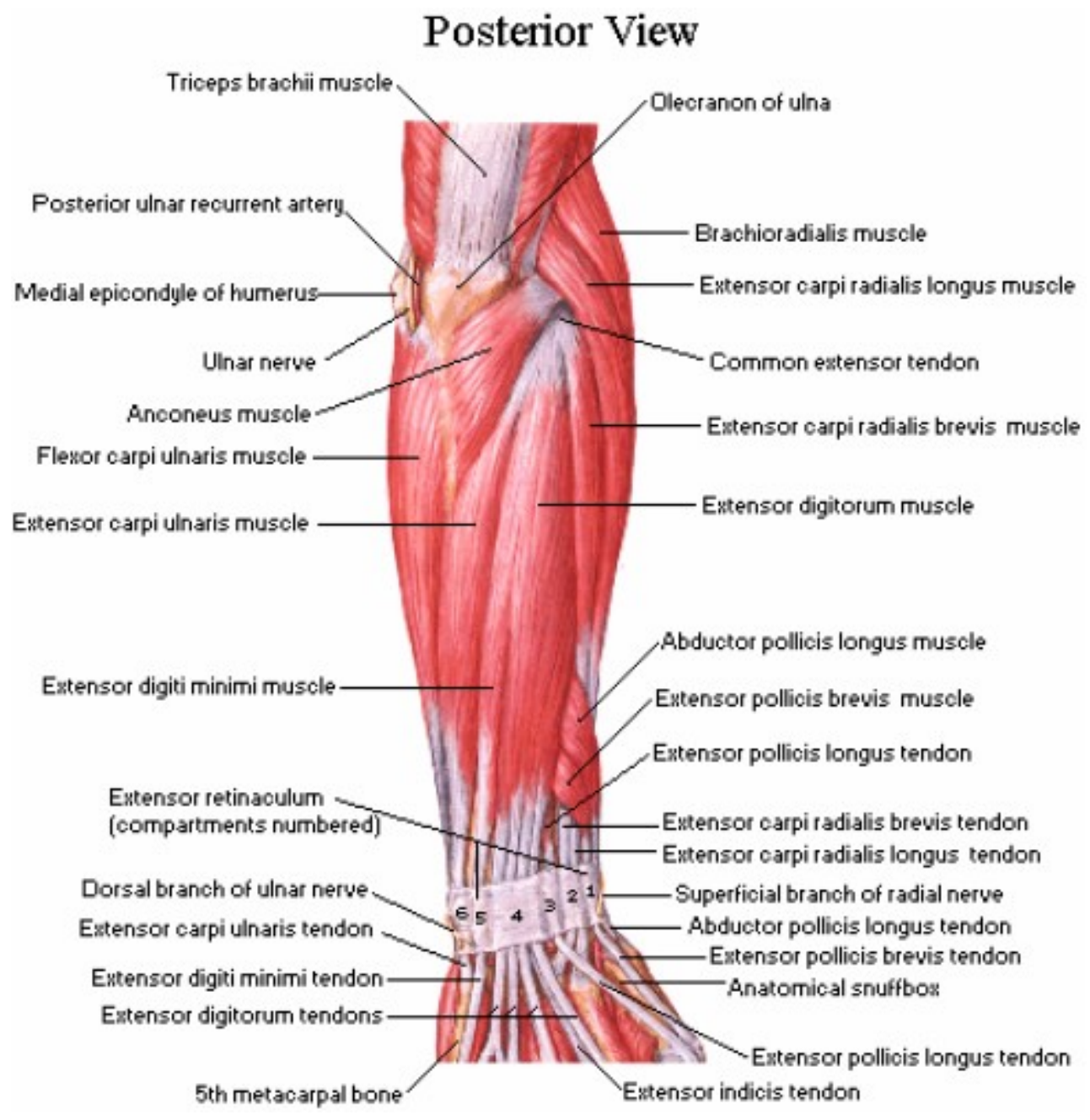

Figure 5.3: Muscle structures of the right forearm in posterior view [107]. 
Table 5.1: Forearm's component of tissues in anterior and posterior sides and tissue level [106-109].

\begin{tabular}{|c|c|c|}
\hline Forearm side & Level of the Muscle & Name of the Muscle \\
\hline \multirow{8}{*}{ Anterior } & \multirow{4}{*}{ superficial } & flexor carpi radialis \\
\hline & & palmaris longus \\
\hline & & flexor carpi ulnaris \\
\hline & & pronator teres \\
\hline & $\begin{array}{l}\text { superficial (or } \\
\text { intermediate) }\end{array}$ & $\begin{array}{l}\text { flexor digitorum superficialis } \\
\text { (sublimis) }\end{array}$ \\
\hline & \multirow{3}{*}{ deep } & flexor digitorum profundus \\
\hline & & flexor pollicis longus \\
\hline & & pronator quadratus \\
\hline \multirow{11}{*}{ Posterior } & \multirow{3}{*}{ superficial } & extensor carpi radialis longus \\
\hline & & extensor carpi radialis brevis \\
\hline & & extensor carpi ulnaris \\
\hline & \multirow{2}{*}{ intermediate } & extensor digitorum (communis) \\
\hline & & extensor digiti minimi (proprius) \\
\hline & \multirow{6}{*}{ deep } & abductor pollicis longus \\
\hline & & extensor pollicis brevis \\
\hline & & extensor pollicis longus \\
\hline & & extensor indicis (proprius) \\
\hline & & supinator \\
\hline & & anconeus \\
\hline
\end{tabular}


As seen in Table 5.1, there are three levels of depth of muscles in each side of the forearm: superficial, intermediate, and deep. The posterior side is mostly composed of extensor muscles. Since the ultrasonic sensor in this research was placed on the posterior side, anterior muscles are excluded from the possible muscles that could be under the ultrasonic sensor. As observed in the in-vivo experiment in Section 5.5, three tissue layers were detected by the ultrasonic sensor between the fat layer and the bone. These three tissue layers explain the three levels of the muscle in the forearm (superficial, intermediate, and deep). Therefore, layers detected from the in-vivo experiment can be defined as follow: $T S_{1}$ (fat), $T S_{2}$ (extensor carpi radialis longus, extensor carpi radialis brevis, or extensor carpi ulnaris), $T S_{3}$ (extensor digitorum, or extensor digiti minimi), $T S_{4}$ (abductor pollicis longus, extensor pollicis brevis, extensor pollicis longus, extensor indicis, supinator, or anconeus), and $T S_{5}$ (radius bone). The boundary echoes obtained in the in-vivo experiment (Section 5.5) could also be from blood vessels and/or the radial nerve. Identification of tissue types are beyond the scope this thesis. The tissue types can be determined more accurately using anatomical images taken by a medical ultrasonic imaging machine in the future study.

A wearable ultrasonic sensor was placed on the right proximal forearm close to elbow in a pronation position on the posterior side. Figure 5.4 demonstrates the location of the ultrasonic sensor on the proximal forearm in the pronation position (Figure 5.4a) and in the posterior view with superficial and deep level of muscles (Figures 5.4b and 5.4c respectively). Hence, From Figure 5.4, we can assume that tissues beneath the ultrasonic sensor are extensor digitorum muscle (Figure 5.4c 12), deep branch of radial nerve (Figure 5.4b 18), supinator muscle (Figures 5.4b 17 and a 3), and the radius bone 
(Figure 5.4b 5). The cross-sectional of a MRI image in Figure 5.5 shows the same tissue layer components as in Figure 5.4 (17: extensor digitorum muscle, 16: deep branch of radial nerve, 11: supinator muscle and 1: radius bone) [106]. 
Rotators of Radius - Pronation

a)

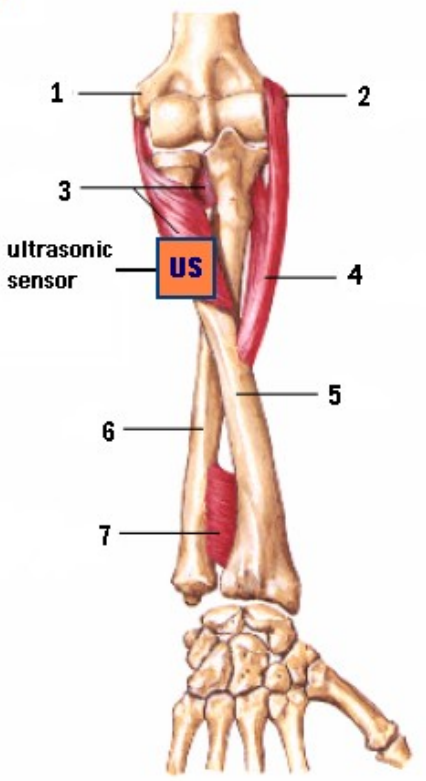

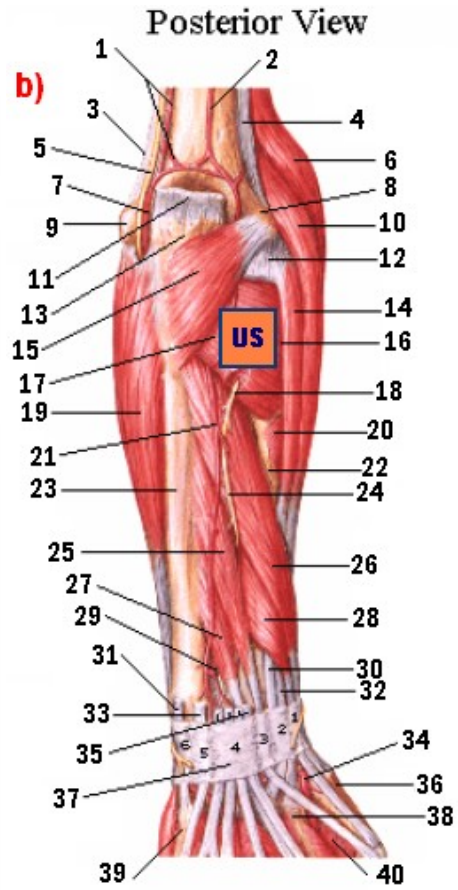

Figure 5.4: a) 1- lateral epicondyle, 2- medial epicondyle, 3- supinator muscle, 4pronator teres muscle, 5- radius, 6- ulna, and 7- pronator quadrates muscle. b) 1superior and inferior ulnar collateral branches of brachial artey, 2- middle collateral

branch of deep brachial artery, 3- medial intermuscular septum, 4- lateral intermuscular septum, 5- ulnar nerve, 6- brachioradialis muscle, 7- posterior ulnar recurrent artery, 8- lateral epicondyle of humerus, 9- medial epicondyle of humerus, 10extensor carpi radialis longus muscle, 11- triceps brachii tendon (cut), 12-common extensor tendon (partially cut), 13- olecranon of ulna, 14- extensor carpi radialis brevis muscle, 15- anconeus muscle, 16- supinator muscle, 17- interosseous recurrent artery, 18- deep branch of radia nerve, 19- flexor carpi ulnaris muscle, 20-pronator teres muscle (slip of insertion), 21- posterior interosseous artery, 22- radius, 23- ulna, 24posterior interosseous nerve, 25- extensor pollicis longus muscle, 26- abductor pollicis longus muscle, 27- extensor indicis muscle, 28- extensor pollicis brevis muscle, 29anterior interosseous artery (termination), 30- extensor carpi radialis brevis tendon, 31extensor carpi ulnaris tendon (cut), 32- extensor carpi radialis longus tendon, 33extensor digiti minimi tendon (cut), 34- radial artery, 35- extensor digitorum tendons (cut), 36- ${ }^{\text {st }}$ metacarpal bone, 37- extensor retinaculum, 38- $2^{\text {nd }}$ metacarpal bone, 39- $5^{\text {th }}$ metacarpal bone, and 40- $1^{\text {st }}$ dorsal interosseous muscle. c) 1 - triceps brachii muscle, 2olecranon of ulna, 3- posterior ulnar recurrent artery, 4- brachioradialis muscle, 5medial epicondyle of humerus, 6- extensor carpi radialis longus muscle, 7- ulnar nerve, 8- common extensor tendon, 9- anconeus muscle, 10- extensor carpi radialis brevis muscle, 11- flexor carpi ulnaris muscle, 12- extensor digitorum muscle, 13- extensor carpi ulnaris muscle, 14- abductor pollicis longus muscle, 15- extensor digiti minimi muscle, 16- extensor pollicis brevis muscle, 17- extensor retinaculum, 18- extensor pollicis longus tendon, 19- dorsal branch of ulnar nerve, 20- extensor carpi radialis brevis tendon, 21- extensor carpi ulnaris tendon, 22- extensor carpi radialis longus tendon, 23- extensor digiti minimi tendon, 24- superficial branch of radial nerve, 25extensor digitorum tendons, 26- abductor pollicis longus tendon, 27- $5^{\text {th }}$ metacarpal bone, 28- extensor pollicis brevis tendon, 29- extensor indicis tendon, 30- anatomical snuffbox, and 31- extensor pollicis longus tendon [107]. 


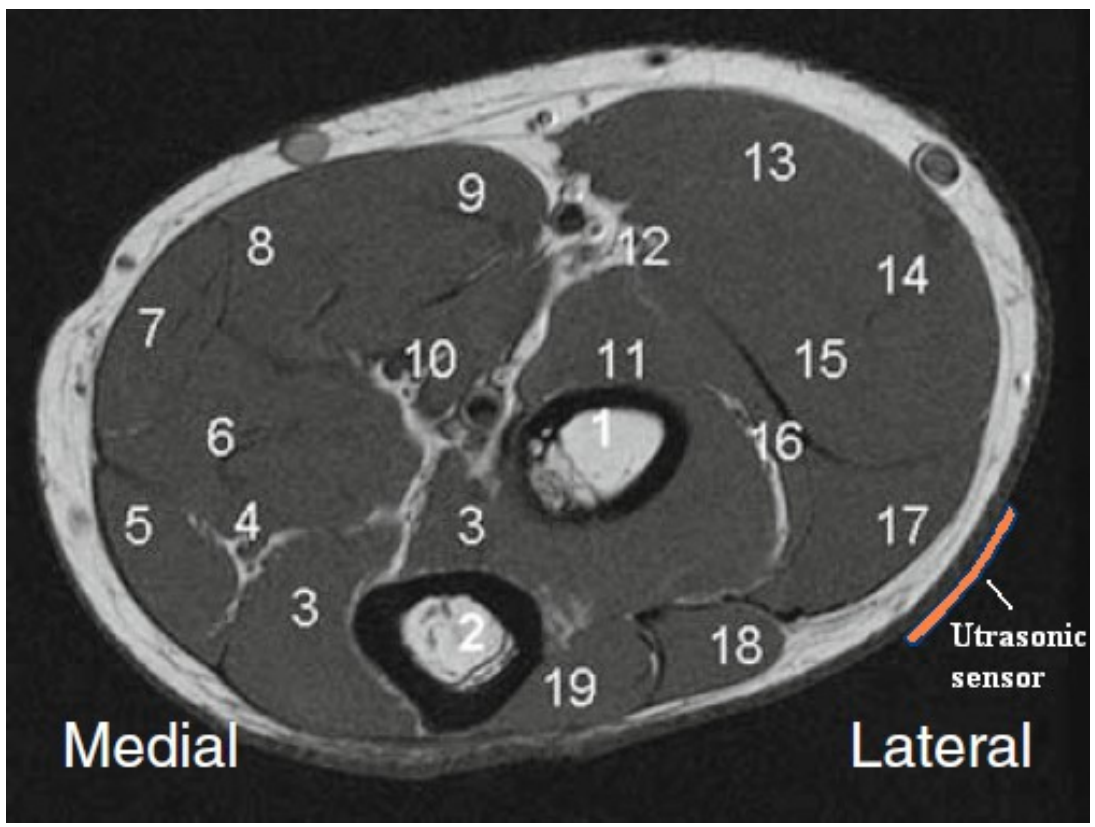

Figure 5.5: Cross-sectional MRI image anatomy of the proximal forearm: 1) radius, 2) ulna, 3) flexor digitorum profundus, 4) ulnar nerve, 5) flexor carpi ulnaris, 6) flexor digitorum superficialis, 7) Palmaris longus, 8) flexor carpi radialis, 9) pronator teres, 10) median nerve, 11) supinator, 12) radial nerve, superficial branch, 13) brachioradialis, 14) extensor carpi radialis longus, 15) extensor carpi radialis brevis, 16) radial nerve, deep branch, 17) extensor digitorum, 18) extensor carpi ulnaris, and 19) anconeus [106].

\subsection{Experimental Tools and Setup}

\subsubsection{Wearable Ultrasonic Sensor}

The conventional ultrasonic probe for medical imaging is bulky, rigid, and heavy.

A rigid and wide ultrasonic probe surface will not be perfectly attached to curvy body surfaces, which may leave gaps between the body surface and some areas of the probe. Moreover, pressing the probe onto the body surface applies force to the underlying 
tissues. Therefore, muscles cannot be freely contracted and their motion may be restricted causing to reduce the measurement accuracy. Furthermore, monitoring the muscle thickness changes often requires body movement by muscle dynamic contractions. It may be difficult to keep a steady and consistent contact between the bulky probe and body surface due to body movement. Hence, it was not suitable to use a conventional ultrasonic probe for muscle monitoring in this research. In additional to these limitations (bulky, rigid, and heavy), the conventional probe is expensive.

To overcome the limitations of the conventional ultrasonic probe mentioned above, we developed a wearable ultrasonic sensor using a piezoelectric polymer [7]. Piezoelectric polymers such as polyvinylidene fluoride (PVDF) are flexible and have acoustic impedance that matches human soft tissues [110]. Moreover, the PVDF is commercially available as a film. Figure 5.6 presents the schematic of the ultrasonic sensor. Figure 5.7 shows photographs of the ultrasonic sensor. The ultrasonic sensor was composed of three materials: the piezoelectric PVDF, the electrodes and the protection films. It had an active area of $15 \mathrm{~mm}$ by $15 \mathrm{~mm}$ which is the overlapped area of the top and bottom electrodes. The PVDF film was $110 \mu \mathrm{m}$ in thickness. The total thickness of the sensor was $200 \mu \mathrm{m}$ and its weight was $0.3 \mathrm{~g}$. Therefore, this ultrasonic sensor could fulfill the requirements in the experiments of muscle monitoring in this research. 


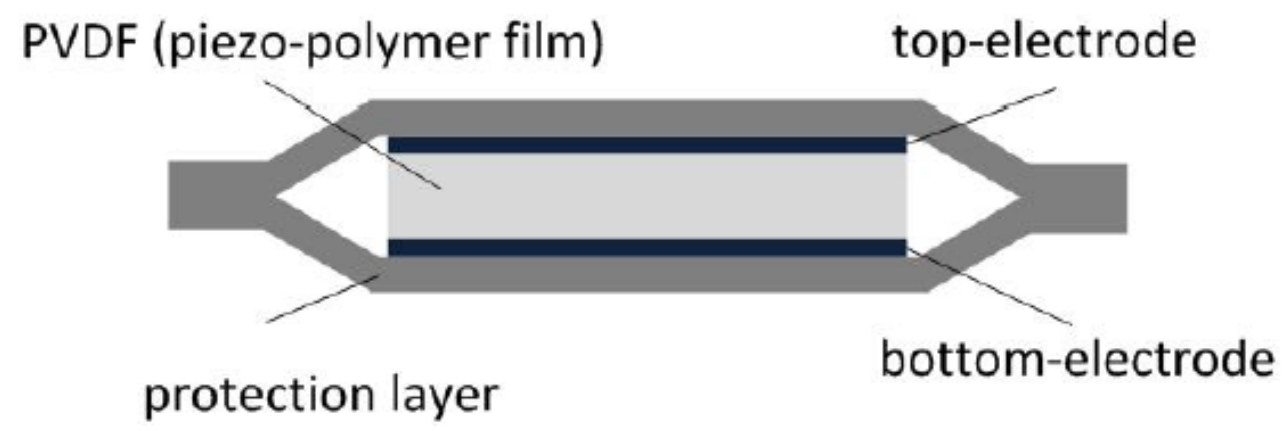

Figure 5.6: Schematic representation of the wearable ultrasonic sensor structure [111].
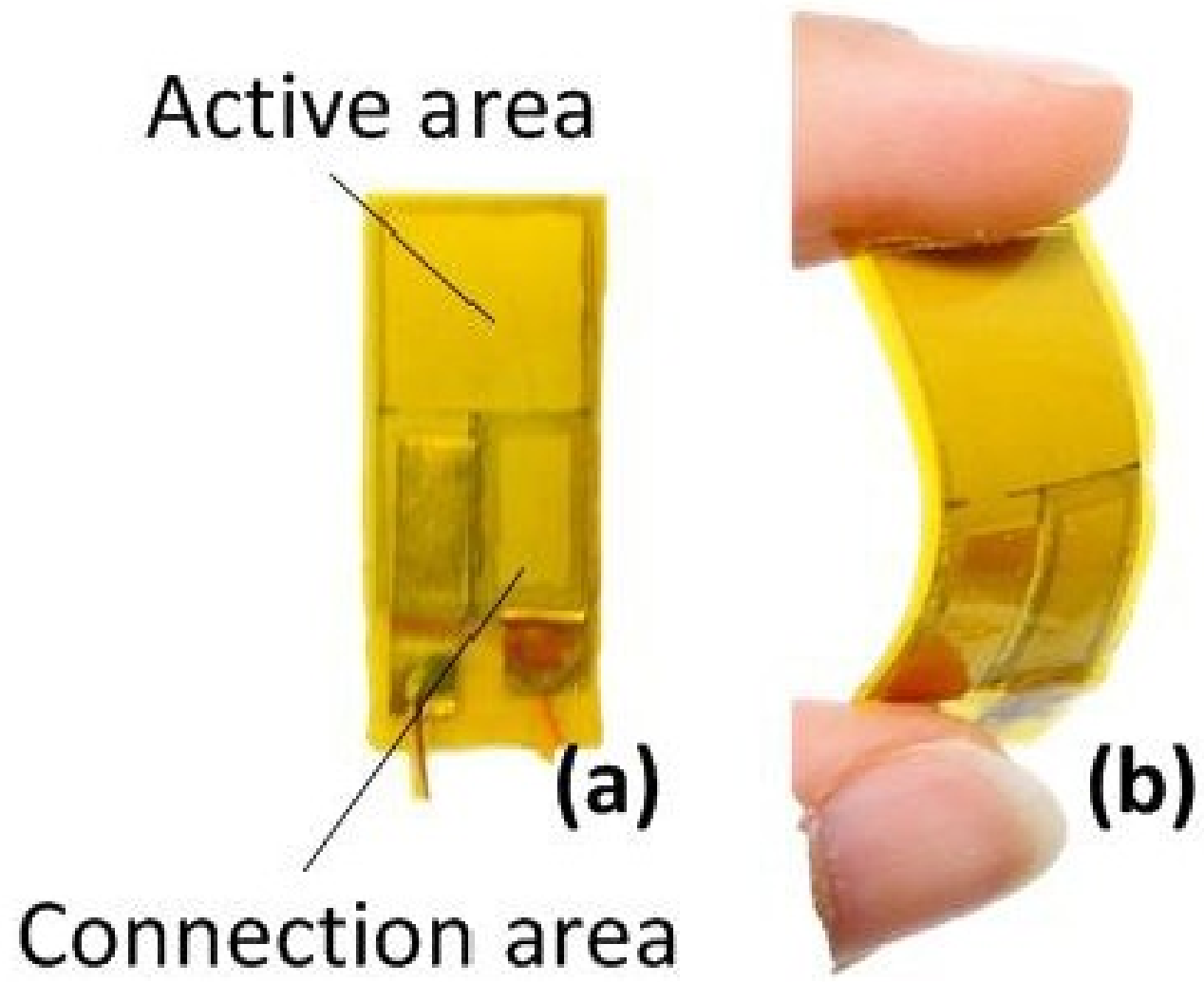

Figure 5.7: Photographs of the wearable ultrasonic sensor. A) top view, and b) side view while bending [111]. 


\subsubsection{Ultrasonic Pulser/Receiver}

Figure 5.8 shows the ultrasonic pulser/receiver (Model 5900PR) from Panametrics-Olympus (Waltham, MA, USA) used in the experiment. The pulser/receiver applies electric pulses to an ultrasonic sensor to generate ultrasound. The pulser/receiver also receives electric signals converted from ultrasound by the ultrasonic sensor.

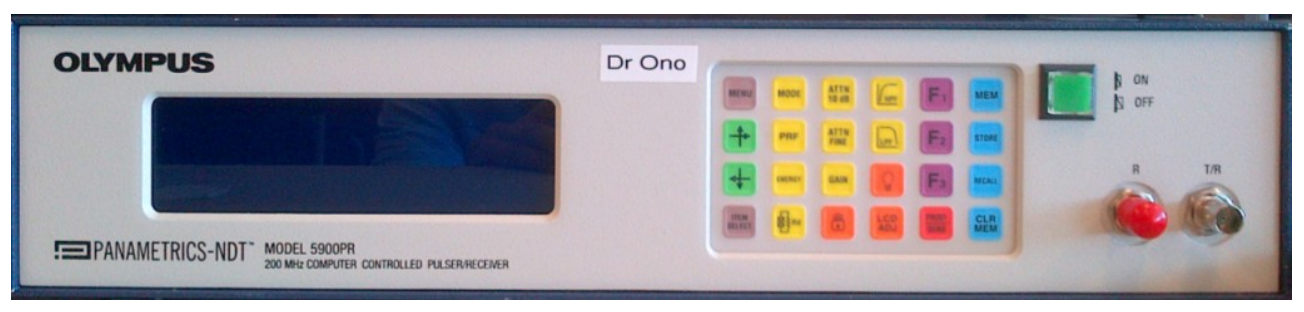

Figure 5.8: The used ultrasonic pulser/receiver device (NDT 5900PR).

\subsubsection{Data Acquisition System}

The data acquisition system contains two parts: PCI digitizer and acquisition program, as shown Figure 5.9. In this research, a PCI digitizer (Model ATS 460) from AlazarTech (Montreal, QC, Canada) was used. It has a 14-bit resolution and a sampling rate range from $10 \mathrm{kHz}$ to $125 \mathrm{MHz}$. An acquisition program was developed using the LABVIEW platform. 


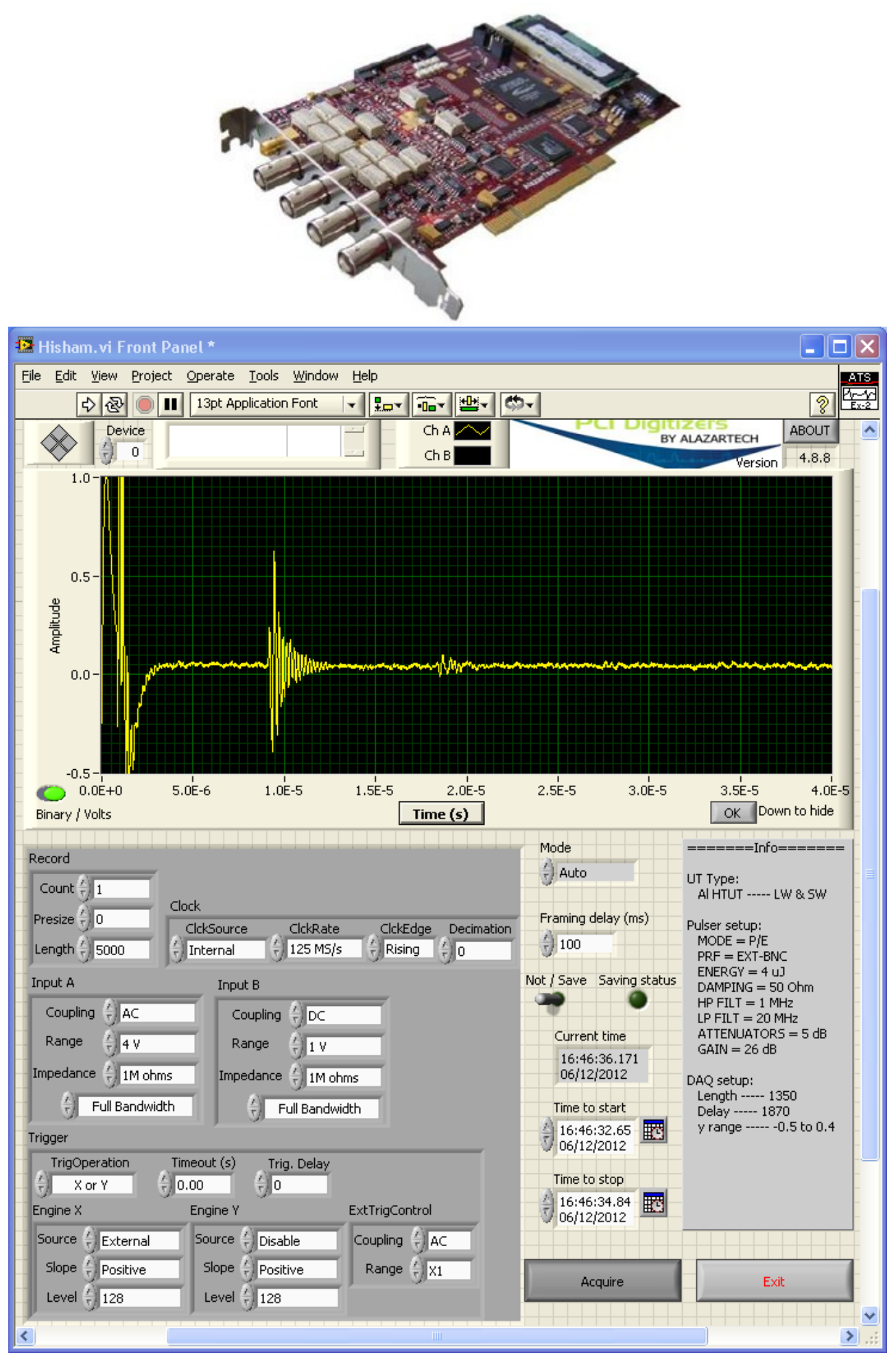

Figure 5.9: a) PCI Digitizer and b) acquisition program. 


\subsubsection{Signal Processing Tool}

MATLAB version 7.12.0.635 (R2011a) was used to analyse the acquired ultrasonic signals. It was also used for the numerical simulation experiments discussed in Chapter 4.

\subsubsection{In-vivo Experimental Setup}

The wearable ultrasonic sensor was attached on the right proximal forearm as seen in Figure 5.10. Figure 5.11 shows a block diagram of the experimental setup. The sensor was connected to the ultrasonic pulser/receiver to transmit and receive the ultrasonic waves. The RF output of the pulser/receiver was connected to the PCI digitizer to correct ultrasonic signals reflected from a body in the ultrasonic pulse-echo mode. As mentioned in Section 5.3, the arm was in the pronation position on the posterior side. .

a)

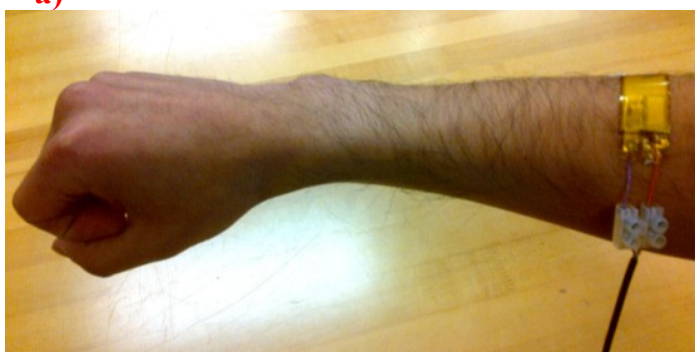

b)

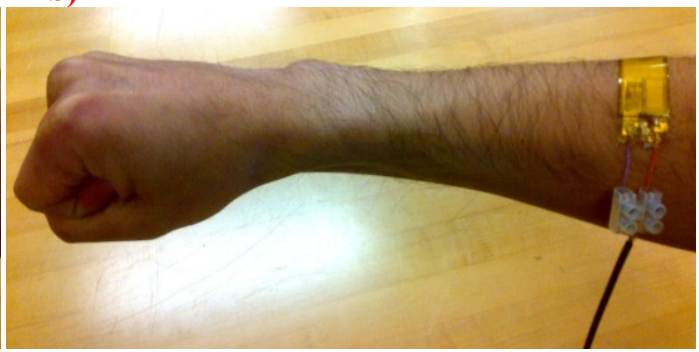

Figure 5.10: Wearable ultrasonic sensor attached on the right proximal forearm a) muscle relax b) muscle isometric contract. 


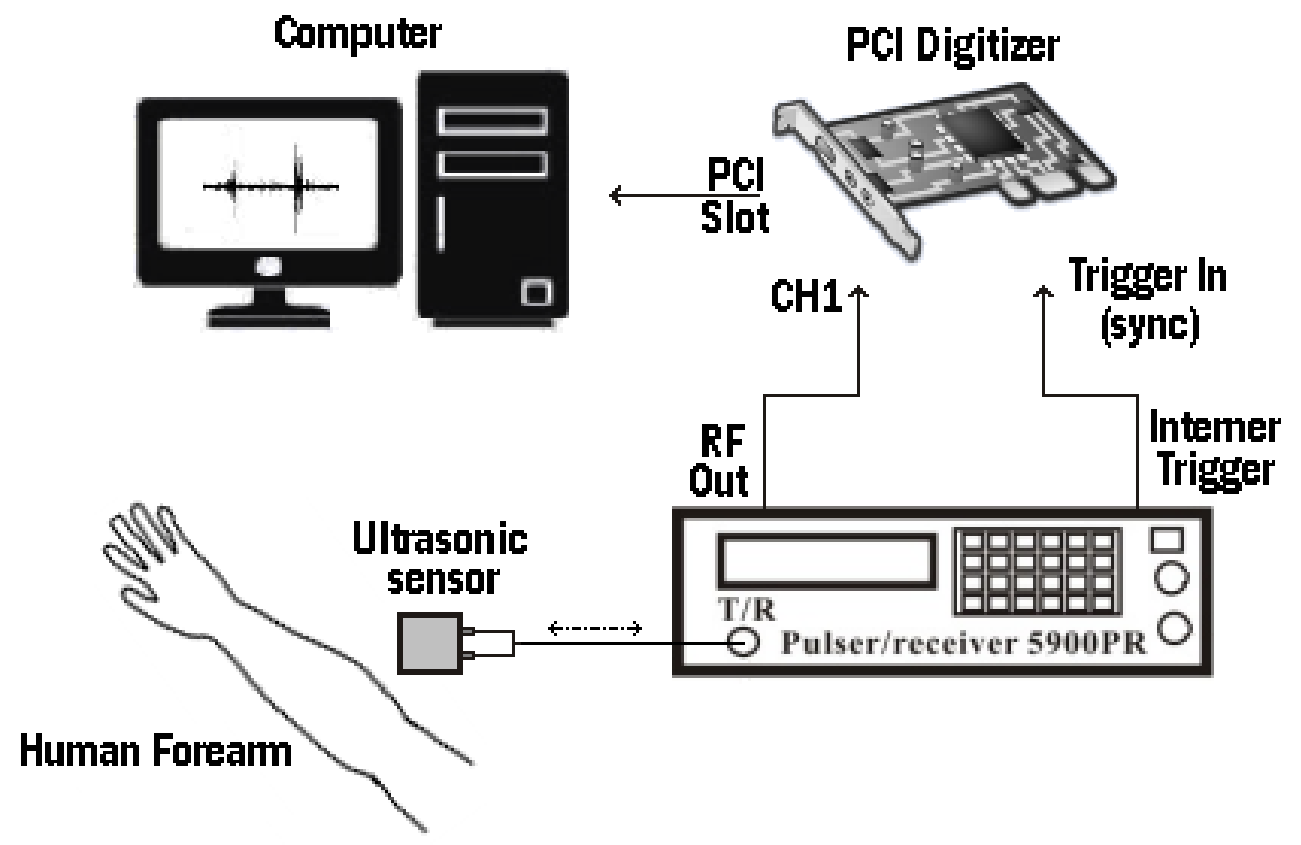

Figure 5.11: The experiment setup in the ultrasonic pulse-echo mode.

\subsubsection{Experiment}

The PCI digitizer was set up to acquire signals with sampling rate of $125 \mathrm{MHz}(8$ $\mu \mathrm{sec}$ ) and frame rate of $10 \mathrm{~Hz}$. The total sampling number of the acquired signals is 5000 points, which corresponds to $40 \mu \mathrm{sec}$. The 5000 sampling points correspond to the total depth of $32.6 \mathrm{~mm}$ with the ultrasound velocity of $1630 \mathrm{~m} / \mathrm{s}$. During the signal acquisition, isometric contraction of the forearm muscles beneath the sensor was performed by clenching and relaxing the right hand alternately for 10 seconds with the transition interval of 2 seconds. The signals were acquired for total 60 seconds in M-mode ultrasound measurement. 


\subsection{In-vivo Experiment Results}

In Chapter 4, the effect of the scattering echoes on the accuracy of tissue boundary motion tracking was investigated using the selected signal processing techniques with three cases of numerical simulation experiments. In this section, the techniques were used to track the tissue boundary motion during the isometric contraction in the in-vivo experiment. Tissue boundary depths were obtained by Equation 5.1. In this section, the subscript $j$ is omitted from the symbols.

\subsubsection{M-mode Ultrasonic Measurement Result}

The tissue boundary motion during the isometric contraction was observed qualitatively with M-mode ultrasonic measurement as seen in Figure 5.12. 


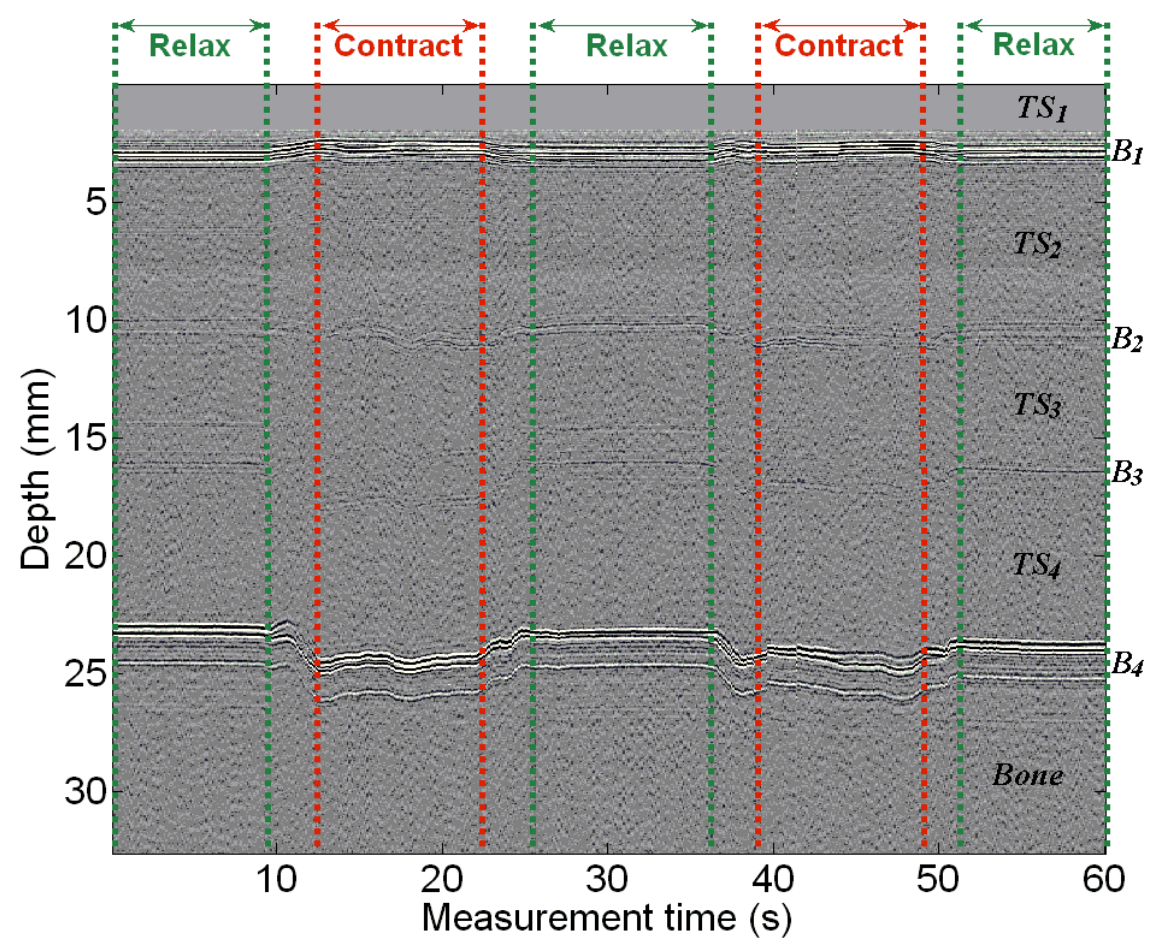

Figure 5.12: The experimental M-mode ultrasonic measurement image, where $T S_{i}$ is the tissue and $B_{i}$ is the tissue boundary.

In Figure 5.12, four boundary echoes, corresponding to tissue boundaries $\left(B_{1}, B_{2}\right.$, $B_{3}$ and $\left.B_{4}\right)$ and tissue layers $\left(T S_{1}, T S_{2}, T S_{3}\right.$ and $\left.T S_{4}\right)$, were observed. The depths of the boundaries changed during the isometric contraction. The relax and contract periods are indicated by the green (0-10 sec, 25-36 sec, and 51-60 sec) and red (12-22 sec and 39-49 sec) dotted lines, respectively. Figure 5.13 presents the acquired raw ultrasonic signals of all frames in $\mathrm{A}$-mode format. It can be seen that the echoes from $\mathrm{B}_{1}$ and $\mathrm{B}_{4}$ have good SNR values, while the echoes from $B_{2}$ and $B_{3}$ have very poor SNR values. The variation in the echo amplitude is because of the variation in the differences between two consecutive tissues acoustic impedance, i.e. the less difference, the less echo amplitude. In the following sections, each boundary echo are analysed to determine its boundary depth change using the signal processing techniques discussed in Chapters 3 and 4. 


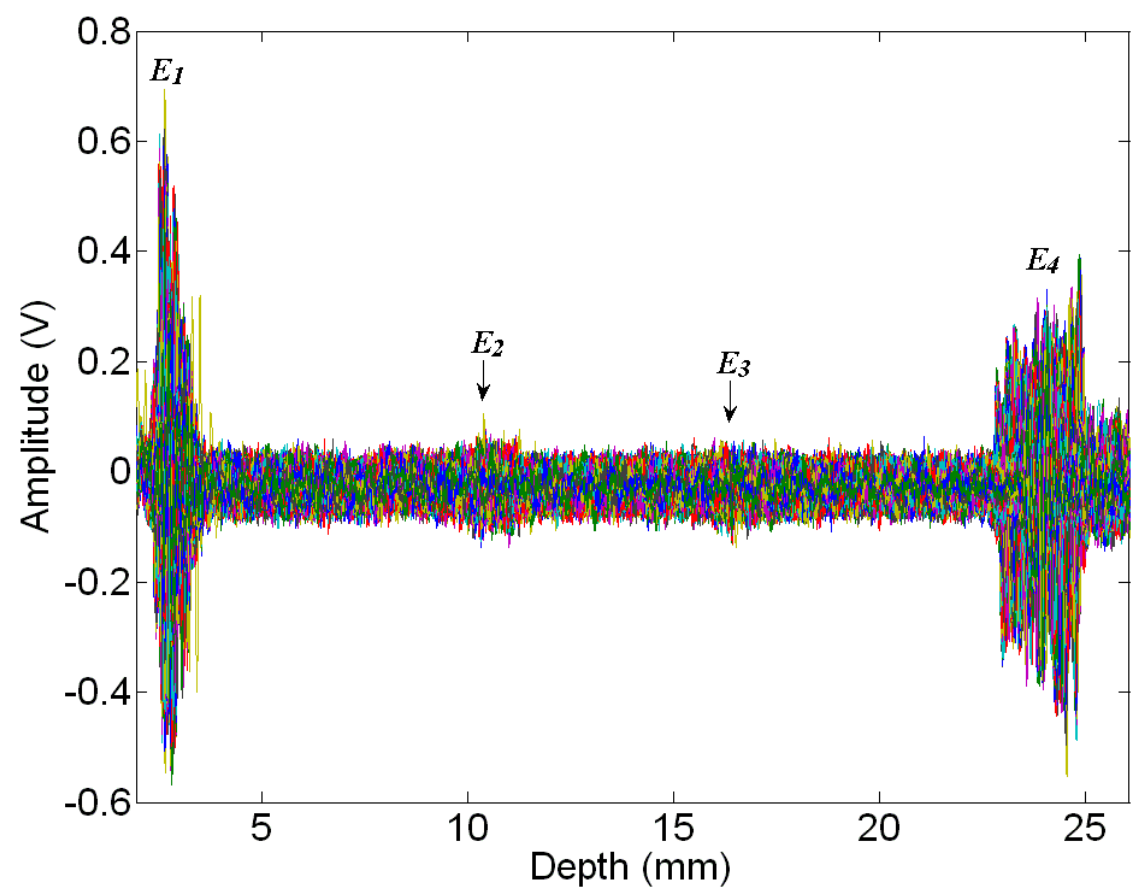

Figure 5.13: All frames raw signal showing the location of the tissue boundary echoes.

\subsubsection{Boundary \#1}

Figure 5.14 shows the M-mode image of the first boundary echo from $B_{1}$. 


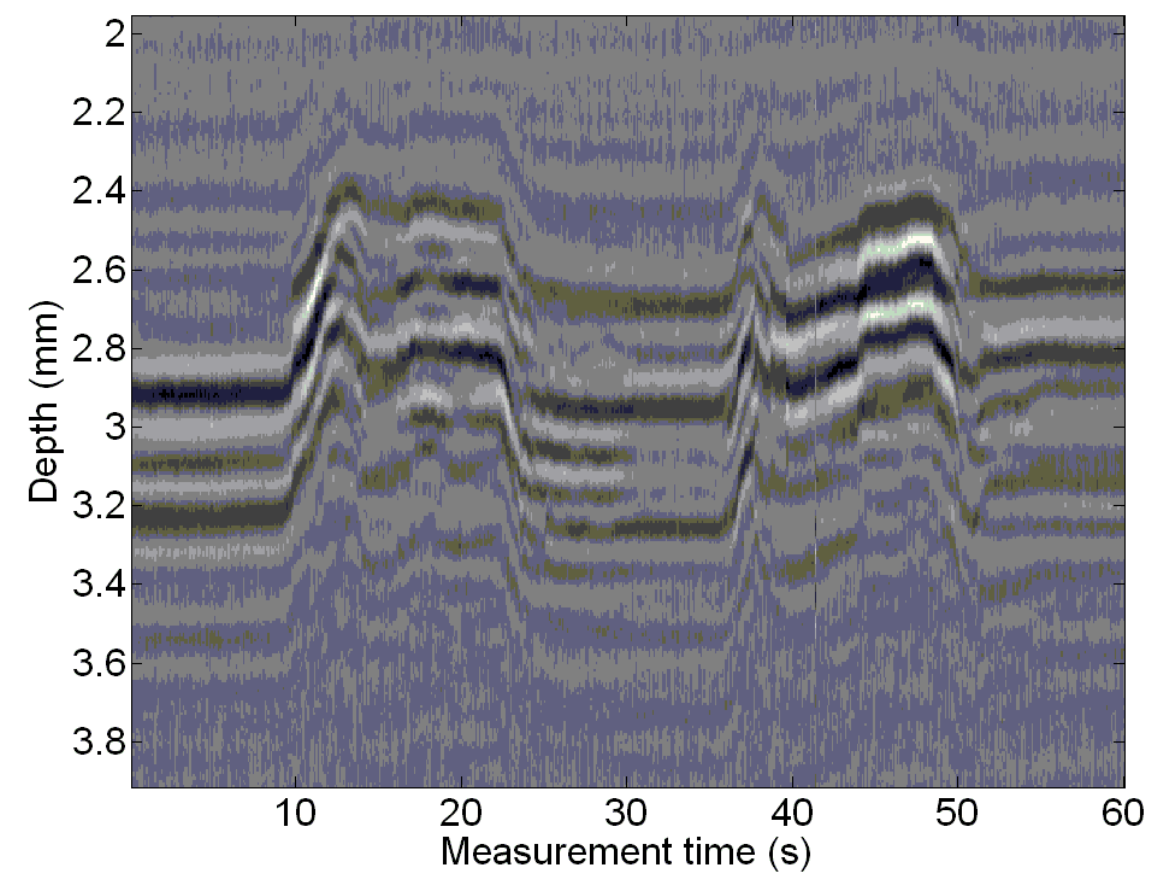

Figure 5.14: Boundary echo from $B_{1}$ in M-mode image.

As observed from Figure 5.12, the $B_{1}$ boundary depth was decreasing from $12 \mathrm{sec}$ to $22 \mathrm{sec}$ and from $39 \mathrm{sec}$ to $49 \mathrm{sec}$ during the contraction states. Since the first boundary depth $B_{1}$ variation is equivalent to the first tissue layer thickness $\left(T H_{1}\right)$ changes, this layer thickness decreased during the contraction. This tissue layer is probably composed of the skin and fat. It was squeezed by the muscle layers of which thickness increased during the contraction. In order to get the boundary depth changes quantitatively, ultrasonic echoes were analysed using the signal processing techniques discussed in Chapters 3 and 4.

To apply the techniques of the single frequency phase detection, mean of narrow band frequencies phase detection, and LQ-factorization, the frequency spectra of the first echoes from $B_{1}$ was obtained by discrete fast Fourier transform. The center frequency $(f c)$ 
was found to be $5.034 \mathrm{MHz}$ as shown in Figure 5.15. The narrow bandwidth was selected in the range between $2.936 \mathrm{MHz}(f l)$ and $7.131 \mathrm{MHz}(f h)$, which includes eleven frequencies with the center frequency in the middle.

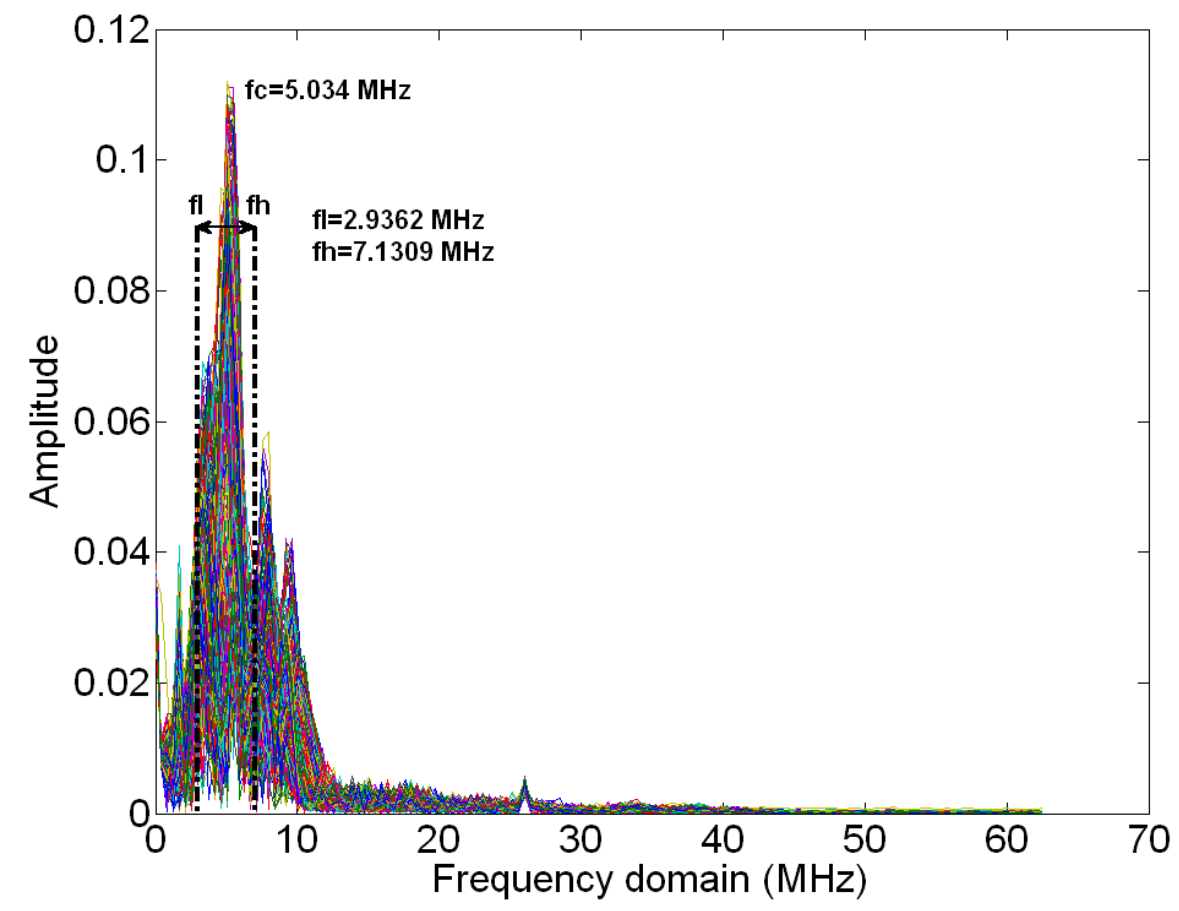

Figure 5.15: Frequency spectrum of $E_{1}$, where $f l$ is the low frequency of the narrow band, $f h$ is the high frequency, and $f c$ is the center frequency.

Figure 5.16 presents the results of the depth of the boundary $B_{1}$. It was observed that cross-correlation of two consecutive frames (c), single frequency phase detection (d), mean of narrow band frequencies phase detection (e), and LQ-factorization techniques (f) tracked the echo boundary $B_{1}$ motion better than a) echo peak detection and b) crosscorrelation between first frame and all frames. 


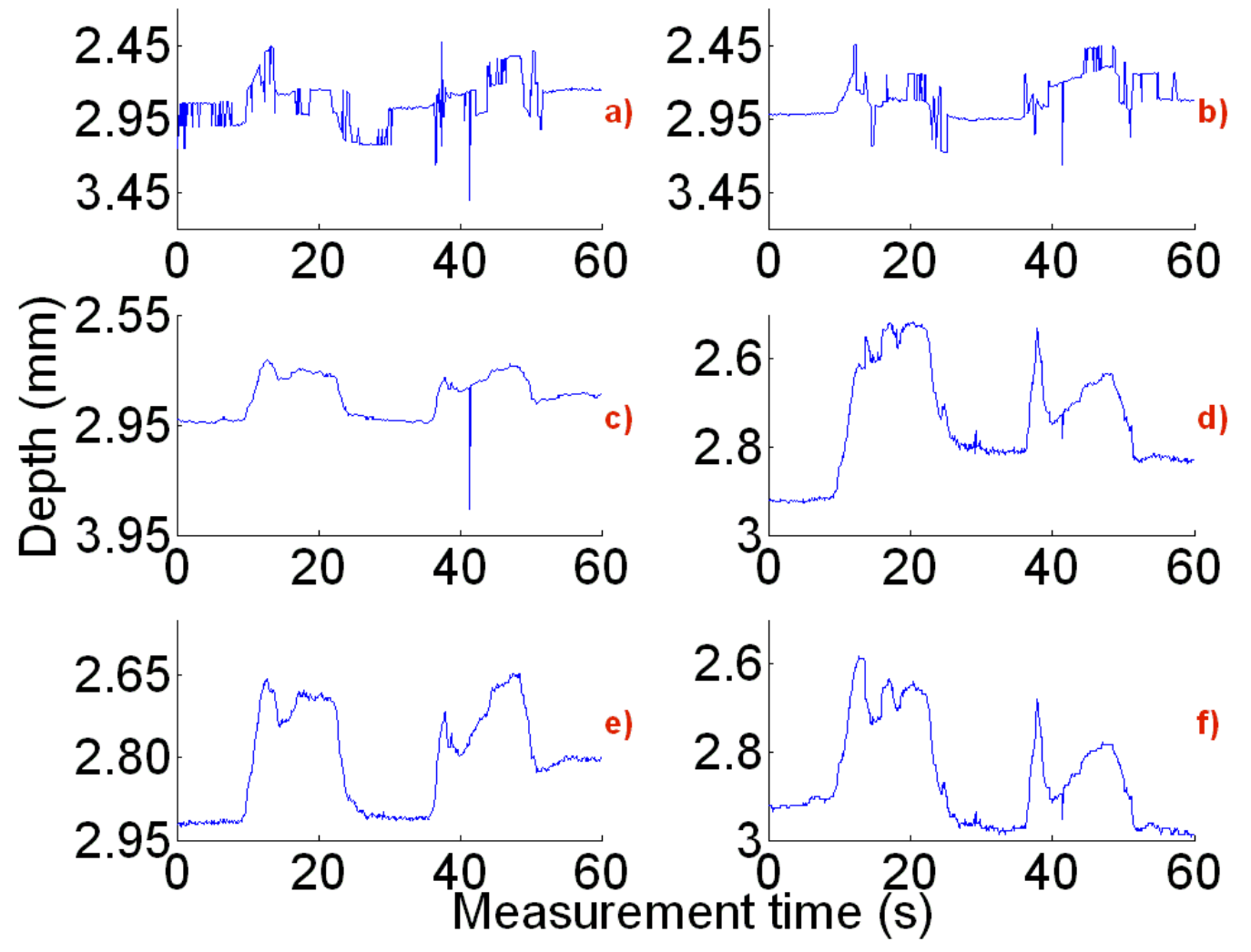

Figure 5.16: Depth of tissue boundary $B_{1}$ obtained by: a) echo peak detection, b) cross-correlation between first frame and all frames, c) cross-correlation between

two consecutive frames, $d$ ) phase detection using single frequency, e) phase detection using the mean of narrow band frequencies, and f) LQ-factorization.

\subsubsection{Boundary \#2}

Boundary echo from $B_{2}$ was difficult to observe in Figures 5.12 and 5.13 because of the low SNR. Figure 5.17 shows the enlarged M-mode image of the boundary echo from $B_{2}$. 


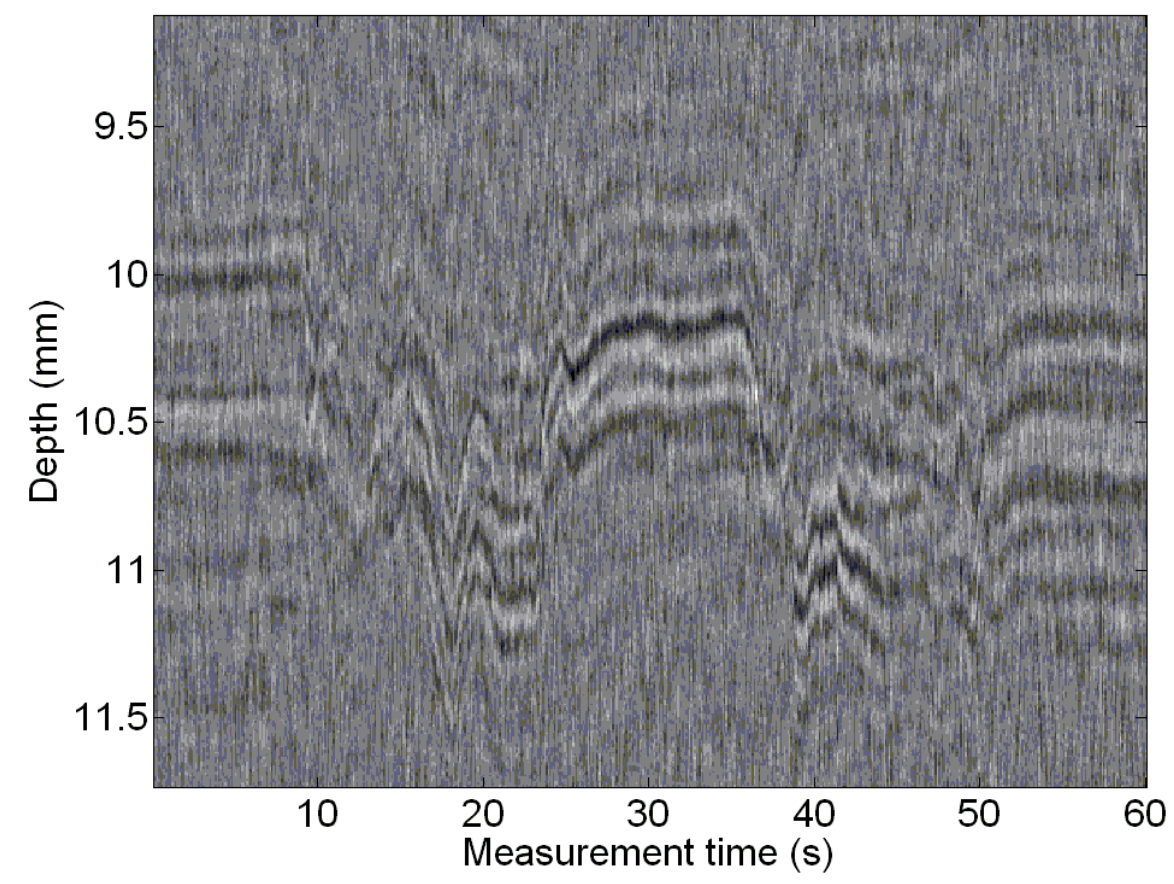

Figure 5.17: Boundary echo from $B_{2}$ in M-mode image.

From Figure 5.17, it can be seen that the depth of $B_{2}$ was increased during the contract states from $12 \mathrm{sec}$ to $22 \mathrm{sec}$ and from $39 \mathrm{sec}$ to $49 \mathrm{sec}$. The thickness of the second tissue layer $\left(T S_{2}\right)$ will be calculated and discussed in Section 5.5.7.

The frequency spectra of the echoes from $B_{2}$ are presented in Figure 5.18. The center frequency was found to be $5.339 \mathrm{MHz}$. The narrow bandwidth was selected in the range between $3.769 \mathrm{MHz}$ and 6.91 $\mathrm{MHz}$. The variation between each boundary echo frequency components are because of variations in the acoustic impedance differences of two consecutive tissues and the depth of each boundary tissue where high frequency waves attenuated more than lower frequency waves for a given distance. 


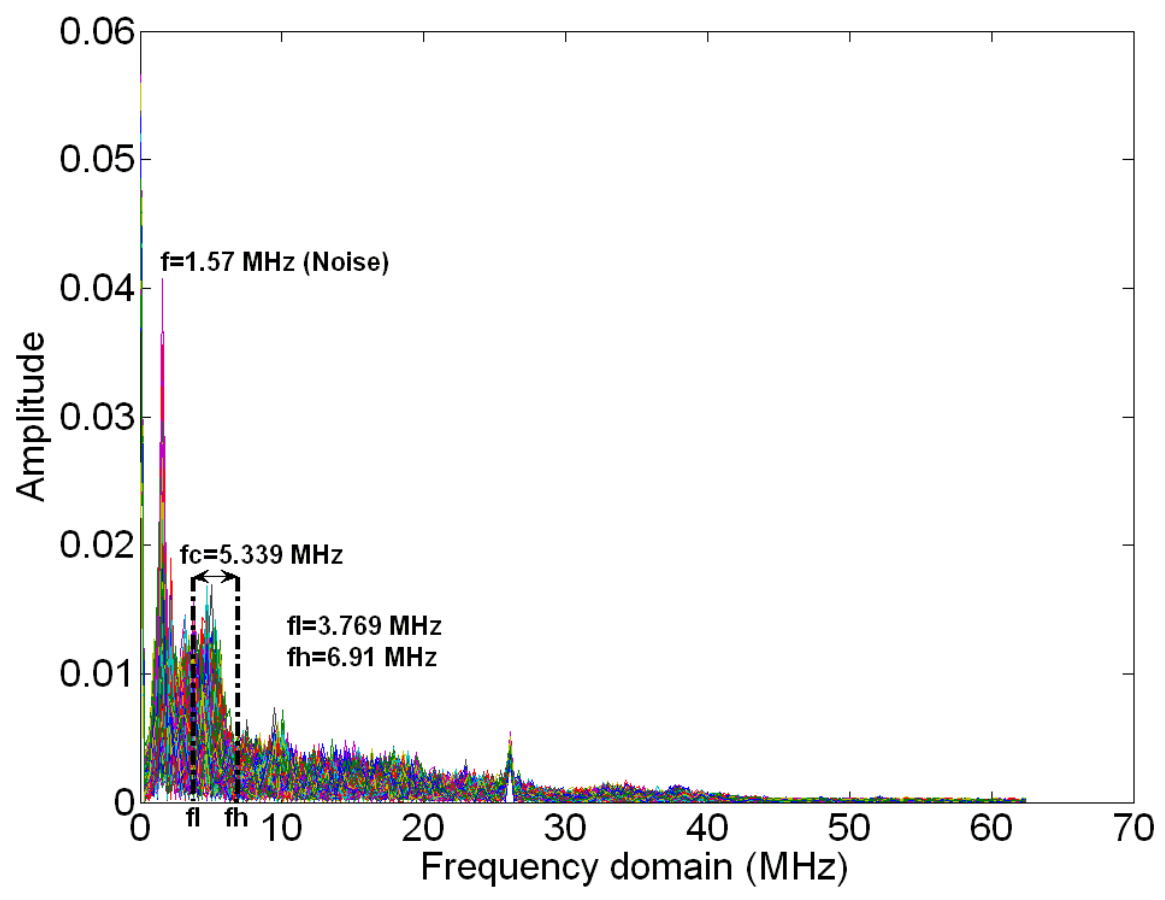

Figure 5.18: Frequency spectrum of $E_{2}$.

In Figure 5.18, a frequency of $1.57 \mathrm{MHz}$ appeared with the greatest amplitude. It was identified as electrical noises from electrical wire since it appeared even when the ultrasonic sensor was disconnected.

Unlike the results for the boundary $B_{1}$ shown in Figure 5.16, cross-correlation between two consecutive frames c) and mean of narrow band frequencies phase detection techniques e) in Figure 5.19 were not able to track the $B_{2}$ motion, while the single frequency phase detection d) and LQ-factorization f) tracked it successfully. 


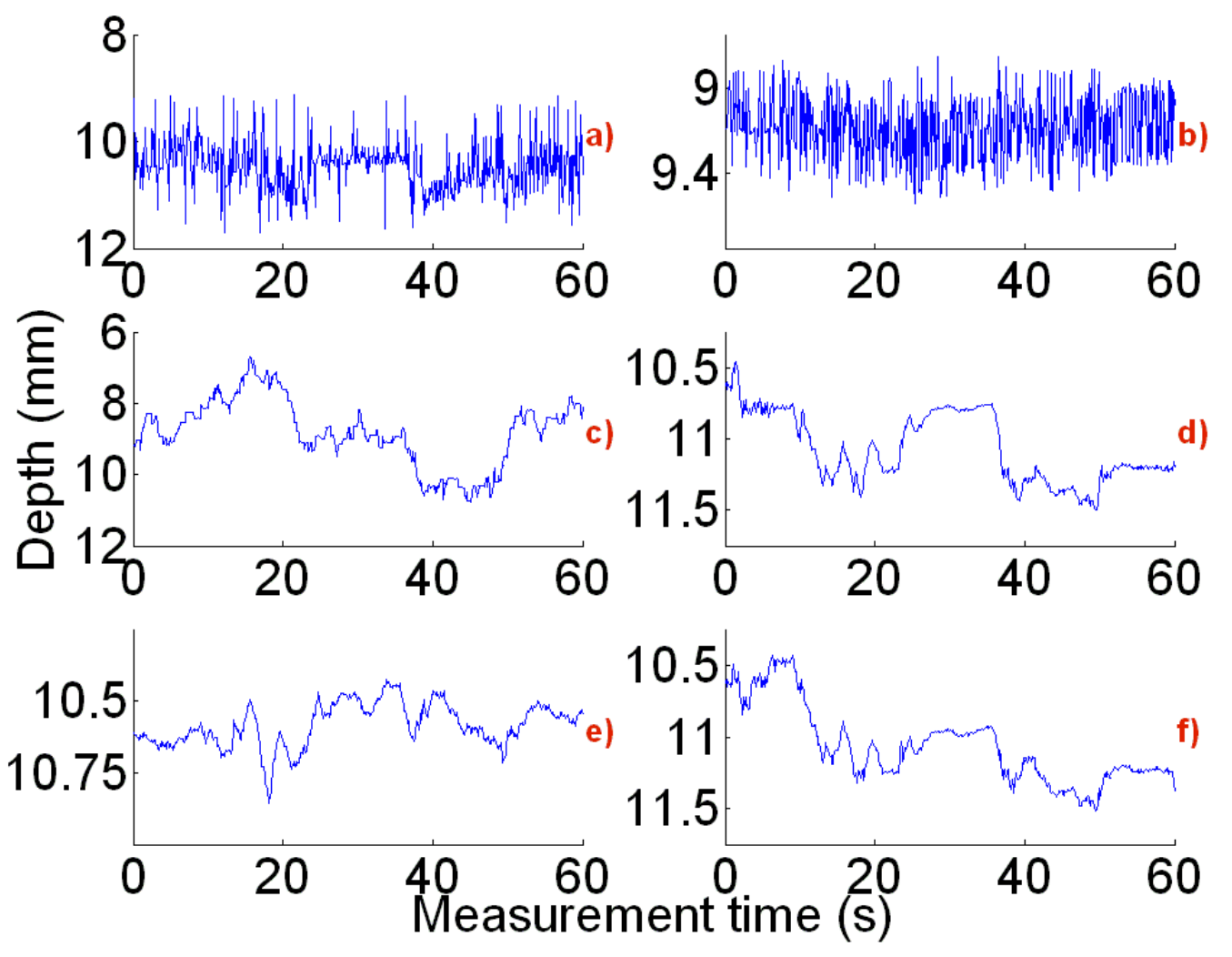

Figure 5.19: Depth of tissue boundary $B_{2}$ obtained by: a) echo peak detection, b) cross-correlation between first frame and all frames, c) cross-correlation between two consecutive frame, $d$ ) single frequency phase detection, e) mean of narrow band frequencies phase detection, and LQ-factorization techniques.

\subsubsection{Boundary \#3}

Boundary echo from $B_{3}$ has the weakest signal amplitude among all boundaries.

Figure 5.20 shows the M-mode format of $B_{3}$. 


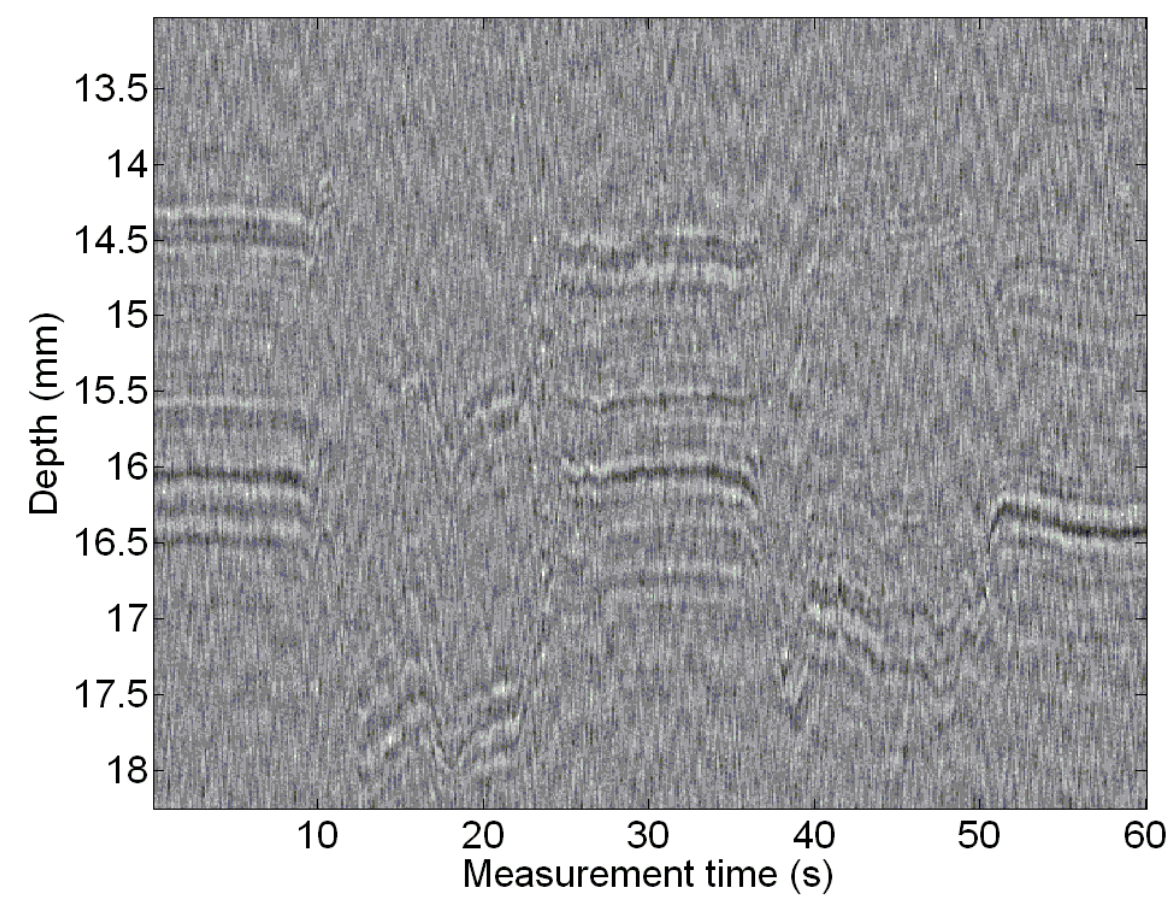

Figure 5.20: Boundary echo from $B_{3}$ in M-mode image.

From the M-mode image above, it was clear that the boundary depth was increasing from $12 \mathrm{sec}$ to $22 \mathrm{sec}$ and from $39 \mathrm{sec}$ to $49 \mathrm{sec}$. The third tissue thickness $\left(T S_{3}\right)$ will be calculated and discussed in Section 5.5.7.

As shown in Figure 5.21, the center frequency is $4.229 \mathrm{MHz}$. The narrow bandwidth of the mean of narrow band frequencies phase detection technique was in the range between $3.446 \mathrm{MHz}$ and 5.013 $\mathrm{MHz}$. 


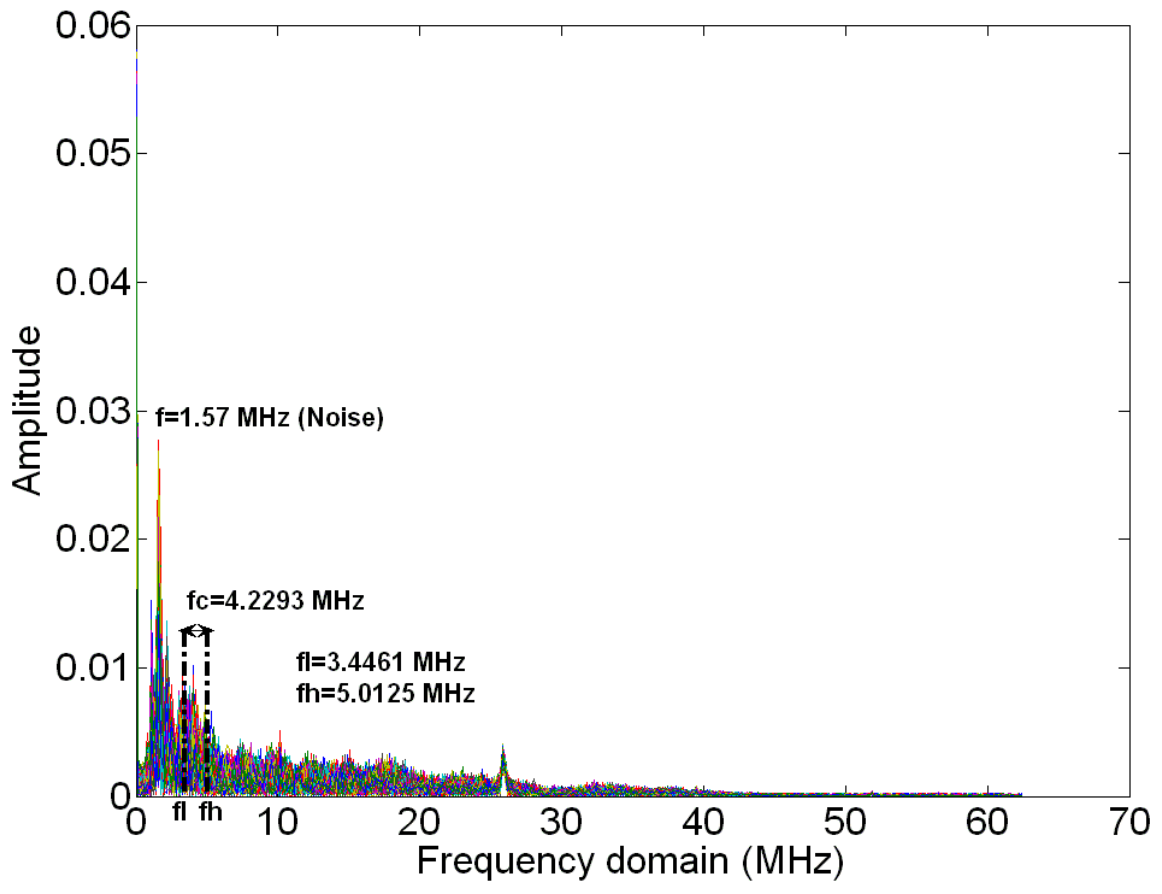

Figure 5.21: Frequency spectrum of $E_{3}$. 


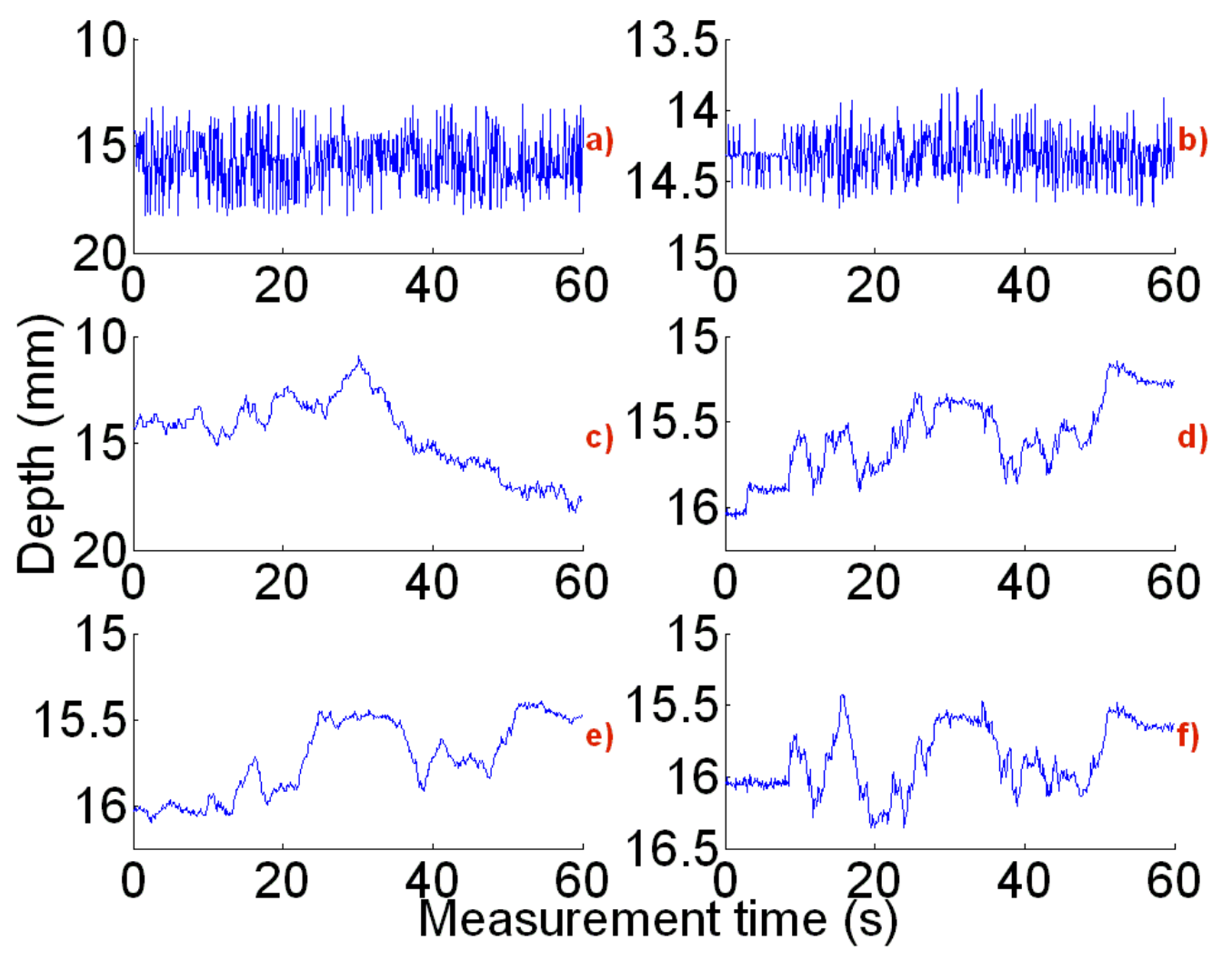

Figure 5.22: Depth of tissue boundary $B_{3}$ obtained by: a) echo peak detection, b) cross-correlation between first frame and all frames, c) cross-correlation between two consecutive frame, $d$ ) single frequency phase detection, e) mean of narrow band frequencies phase detection, and LQ-factorization techniques.

From Figure 5.22, d) single frequency phase detection, e) mean of narrow band frequencies phase detection, f) LQ-factorization techniques successfully tracked the $B_{3}$ motion compared to the M-mode image in Figure 5.20. However, the techniques d), e), and f) had difficulties tracking the boundary motion from $0 \mathrm{sec}$ to $18 \mathrm{sec}$. 


\subsubsection{Boundary \#4}

Finally, boundary echo from $B_{4}$ is the echo from the boundary between the fourth tissue and the bone. $B_{4}$ has good SNR as seen in Figure 5.13.

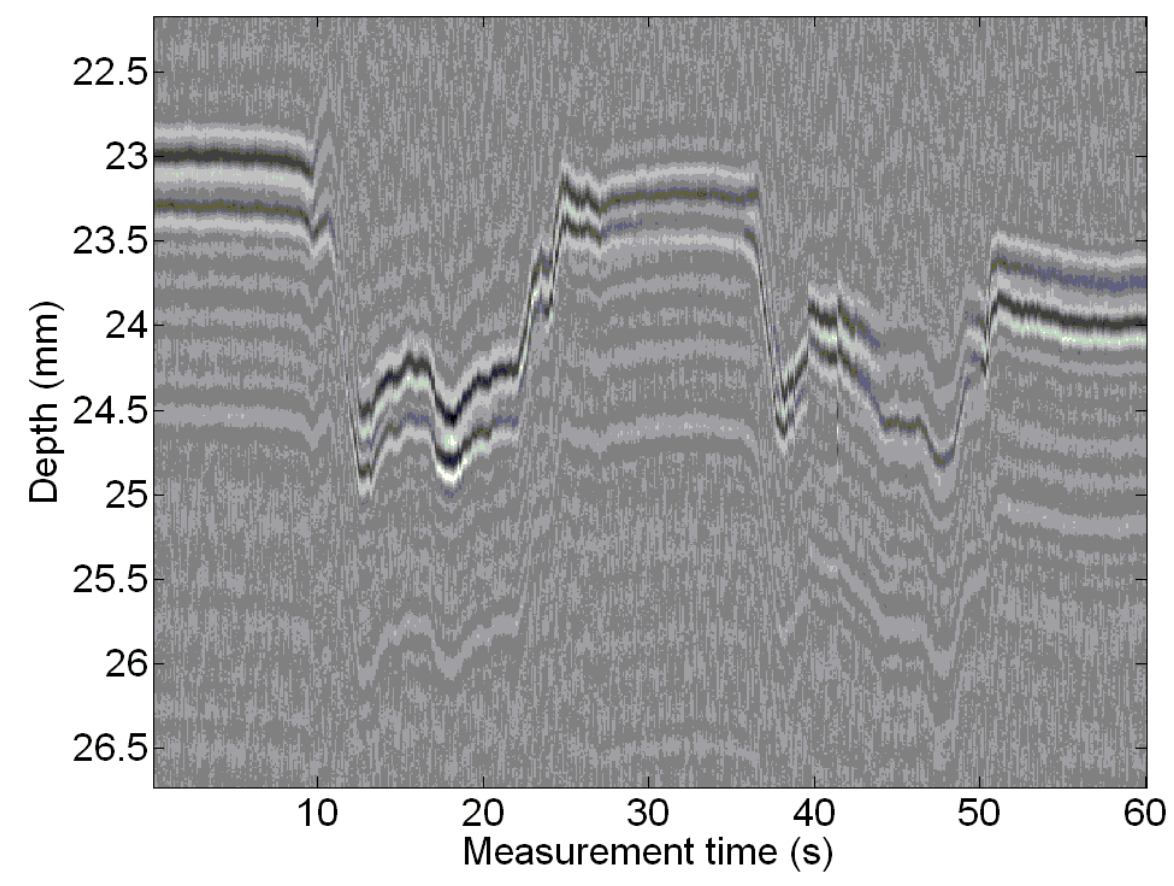

Figure 5.23: Boundary echo from $B_{4}$ in M-mode image.

In Figure 5.23, the depth of the boundary was increasing from $12 \mathrm{sec}$ to $22 \mathrm{sec}$ and from $39 \mathrm{sec}$ to $49 \mathrm{sec}$. The depth of $B_{4}$ is equivalent to the total thickness changes between the ultrasonic sensor and the bone. The thickness of tissue $4\left(\mathrm{TH}_{4}\right)$ and the total thickness between the ultrasonic sensor and the bone $\left(T H_{\text {total }}\right)$ are calculated and discussed in Section 5.5.7. 


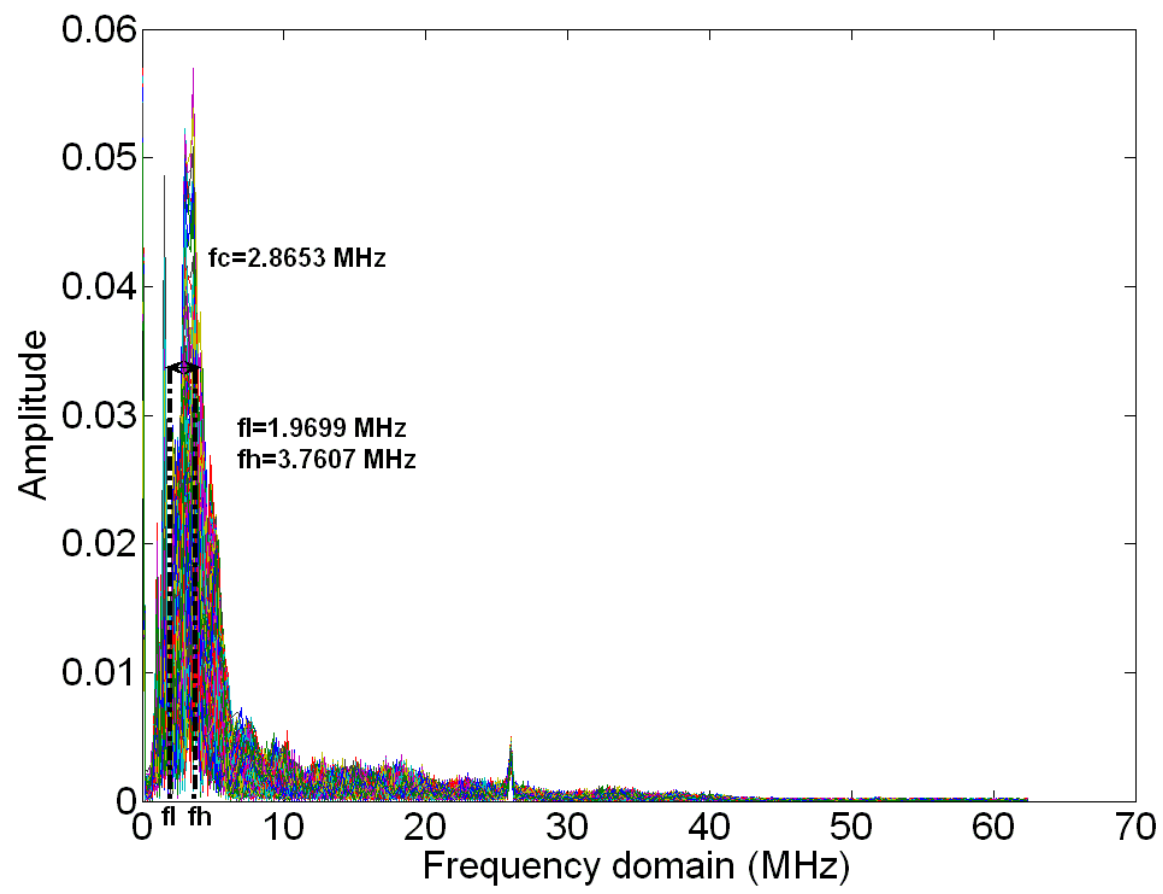

Figure 5.24: Frequency spectrum of $E_{4}$.

The center frequency for $B_{4}$ was $2.865 \mathrm{MHz}$ and the narrow bandwidth was in the range of $1.97 \mathrm{MHz}$ and $3.761 \mathrm{MHz}$, as can be seen in the above figure.

Figure 5.25 represents the results of the displacement of tissue $4\left(T S_{4}\right)$ and the total thickness change as well. All techniques discussed in Chapter 3 have successfully tracked the boundary motion, but with unsmooth tracking in a) echo peak detection and b) cross-correlation between first frame and all frames. 

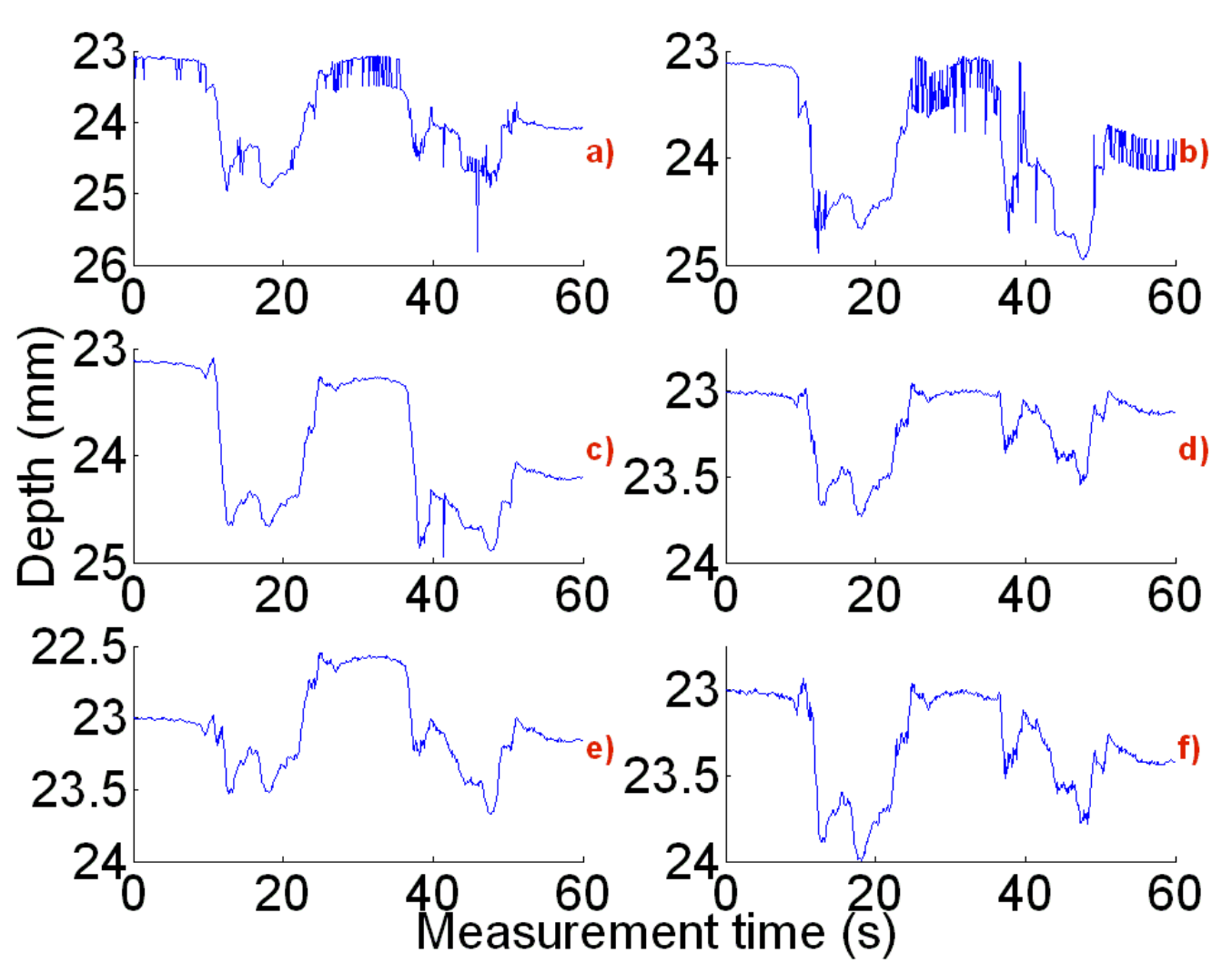

Figure 5.25: Depth of tissue boundary $B_{4}$ obtained by: a) echo peak detection, b) cross-correlation between first frame and all frames, $c$ ) cross-correlation between two consecutive frame, d) single frequency phase detection, e) mean of narrow band frequencies phase detection, and f) LQ-factorization techniques.

\subsubsection{Discussion}

Unlike what was observed from the simulation experiments in Chapter 4, crosscorrelation between two consecutive frames in the in-vivo experiment was more feasible than the cross-correlation between first frame and all frames. A reason for this was probably that the amplitude and waveform of the boundary echoes significantly changes 
during muscle contraction due to the geometrical configuration changes between the sensor and tissue layers in the in-vivo experiment. Hence, the correlation between the first frame and the other frames decreased as measurement time increased.

There were two boundary echoes from $B_{2}$ and $B_{3}$ with very low SNR values. It was very challenging to track these boundary echoes with low SNR. It was verified that the signal processing techniques in frequency domain (the phase detection and the LQfactorization) worked better than those in time domain (the each peak detection and the cross-correlation).

From the in-vivo experiment, the single frequency phase detection and the LQfactorization techniques were able to track reasonably all of the tissue boundary echoes. However, the LQ-factorization was more reliable for the in-vivo experiments as well as for the simulation experiments conducted in Chapter 4 with low RMSE values at all SNR values investigated in this study. Therefore, it is confirmed that the LQ-factorization technique was most accurate in tracking the boundary motion among the techniques investigated in this study.

\subsubsection{Thickness Measurements}

The LQ-factorization technique was proved effective in tracking the boundary echo. Hence, the results of boundary displacements obtained by the LQ-factorization technique in Figures 5.16, 5.19, 5.22, and 5.25 were used to calculate each tissue 
thickness variations during muscle contraction. Figure 5.26 shows the depth change of the tissue boundaries of $B_{1}, B_{2}, B_{3}$ and $B_{4}$ obtained by the LQ-factorization technique from Figures 5.16f, 5.19f, 5.22f, and 5.25f.

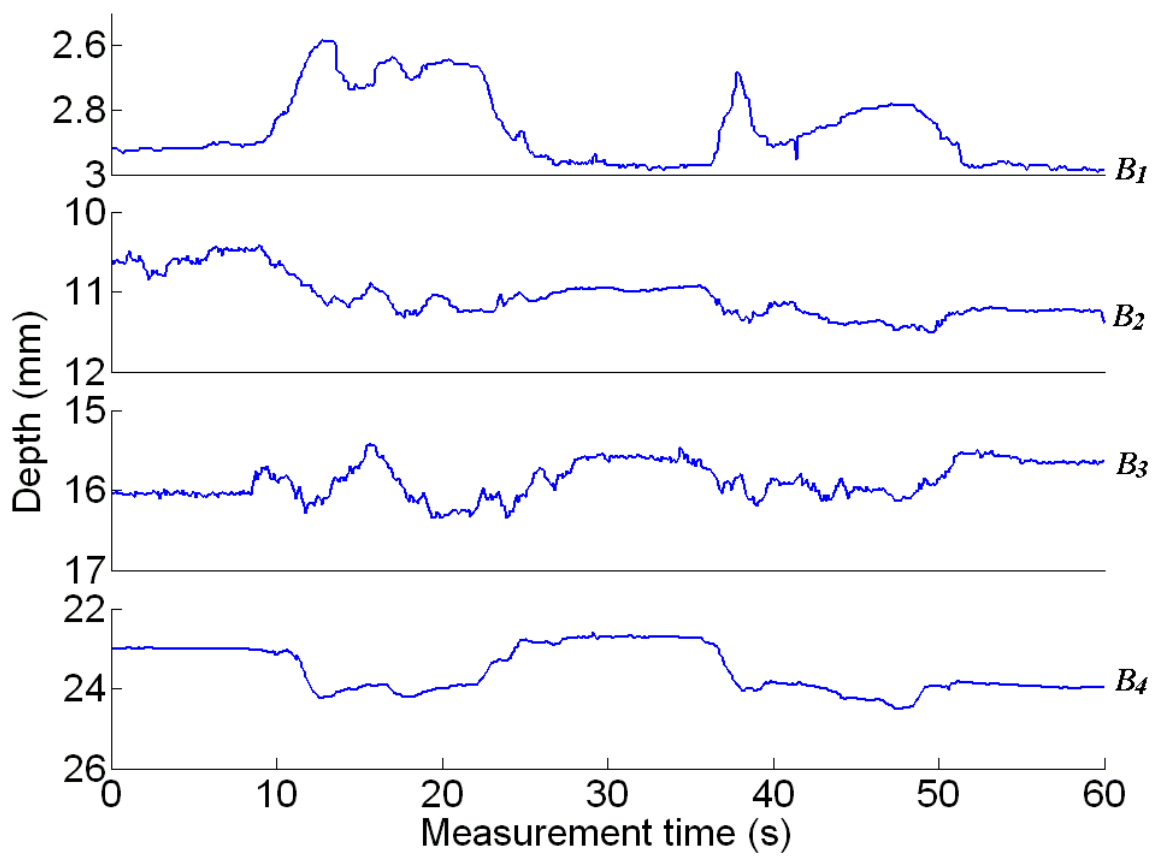

Figure 5.26: Tissue boundary depths obtained by the LQ-factorization technique.

The thickness of each tissue layer was calculated using the results in Figure 5.26 by Equation 3.3. Figure 5.31 presents the thickness changes of the tissue layers of $T S_{1}$, $T S_{2}, T S_{3}$ and $T S_{4}$. 


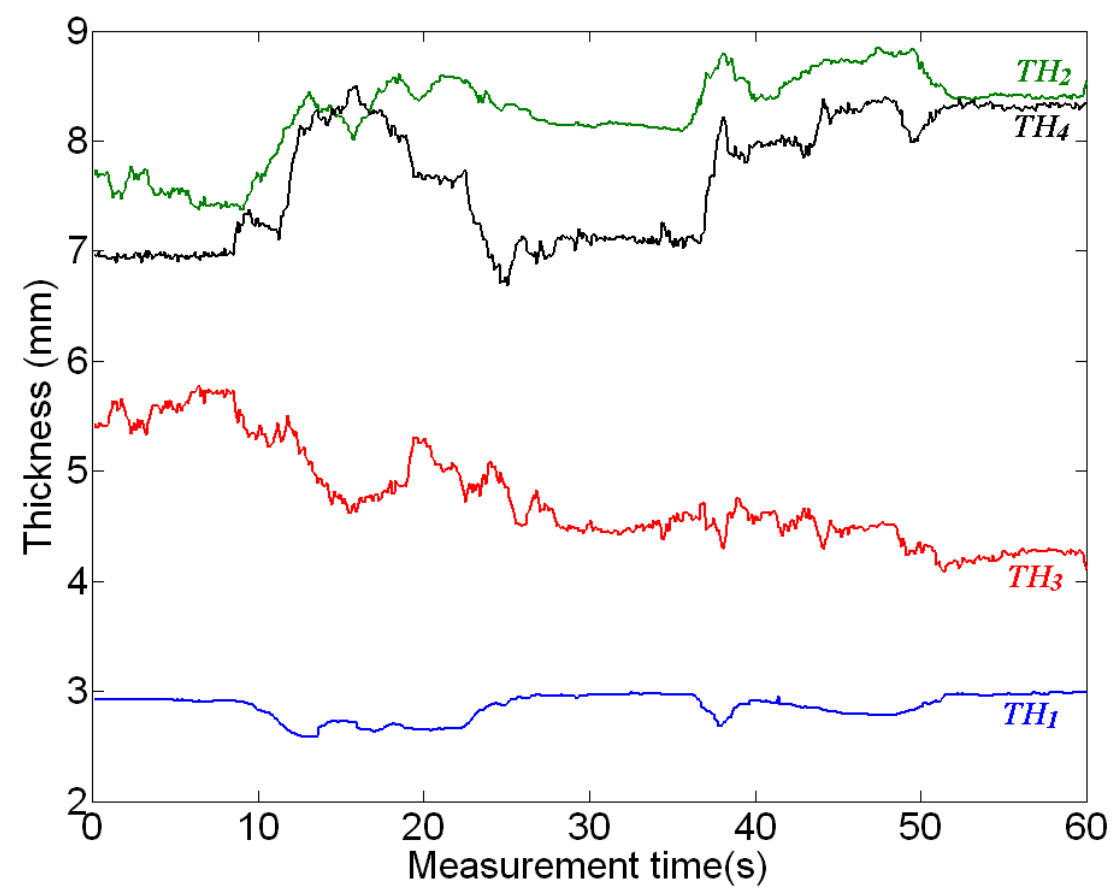

Figure 5.27: Thickness variations of each tissue layer due to muscle contraction.

From Figure 5.27, the thickness of $\mathrm{TH}_{1}$ (skin/fat) decreased during muscle contraction since the increase of the muscle layers caused the $T H_{1}$ to be compressed or squeezed. The thickness change ratio of each tissue was calculated. During the isometric muscle contraction, the thickness of the fat tissue layer $\left(T H_{1}\right)$ decreased by $0.41 \mathrm{~mm}$ (13.7\%). For $\mathrm{TH}_{2}$ and $\mathrm{TH}_{4}$, the total thickness increased by $1.47 \mathrm{~mm}(19.9 \%)$ and 1.81 mm $(27.1 \%)$ for tissue 2 and tissue 4 respectively. It can be observed that $\mathrm{TH}_{3}$ slightly increased during the muscle contraction. However, the total thickness of $\mathrm{TH}_{3}$ was gradually decreasing from $0 \mathrm{sec}$ to $60 \mathrm{sec}$. The reason of the gradual decreases of the $\mathrm{TH}_{3}$ is not clear at the moment. It could be due to the errors in the signal analysis. Further investigation is required to verify this in the future study. The accuracy of the tissue thickness measurement is predicted based on the LQ-factorization RMSE from the third 
numerical simulation experiment in Section 4.2.3. The accuracies are as follows: $1.823 \%$, $1.582 \%, 1.465 \%, 1.008 \%, 0.6025 \%, 0.4711 \%$, and $0.4369 \%$ for $0,5,10,15,20,25$, and $30 \mathrm{~dB}$ SNR respectively.

Figure 5.28 shows the total thickness variations between the ultrasonic sensor and the bone. The total thickness variations $\left(T H_{\text {total }}\right)$ is equivalent to the depth of the boundary located between the fourth tissue $\left(\mathrm{TH}_{4}\right)$ and the bone (Equation 5.4).

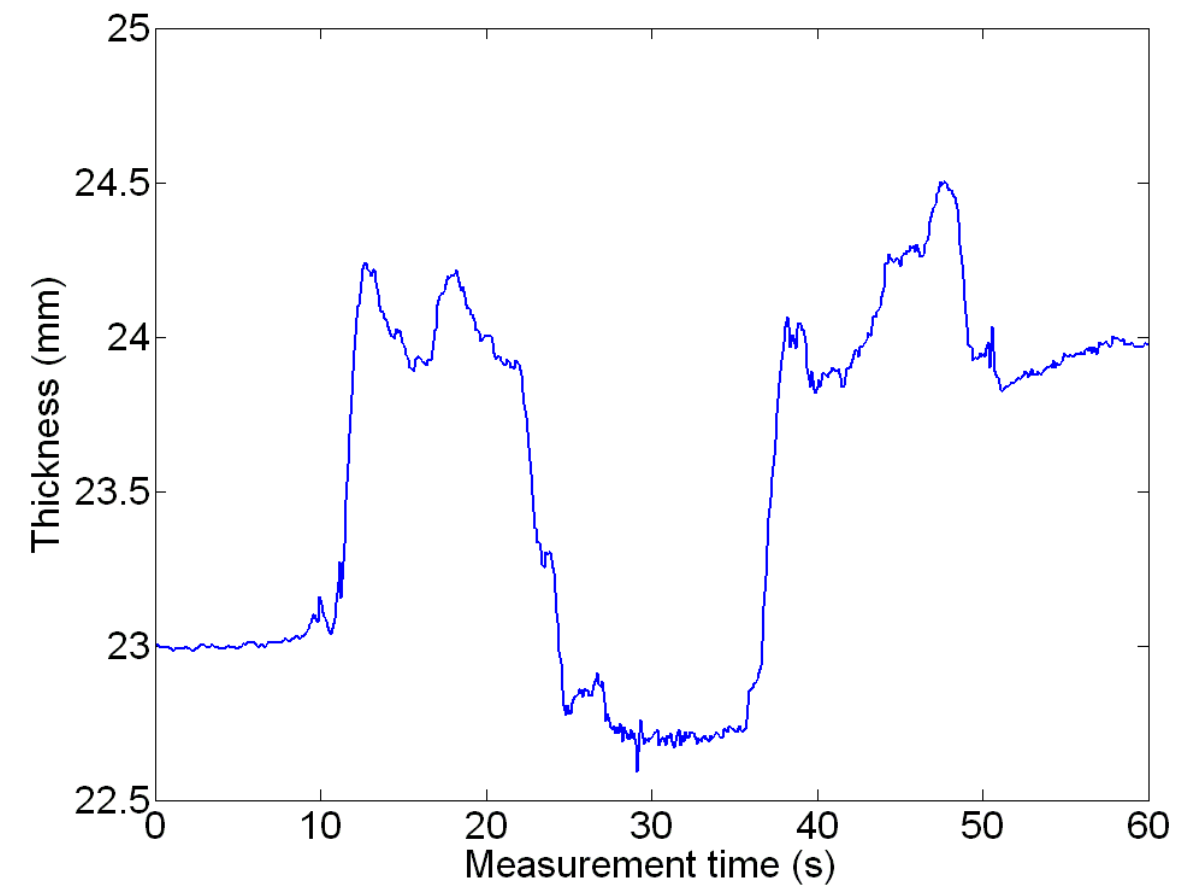

Figure 5.28: Total thickness changes between the ultrasonic sensor and the bone.

The total thickness increased by $0.97 \mathrm{~mm}(4.15 \%)$ during isometric muscle contraction. The largest thickness value of $24.5 \mathrm{~mm}$ was detected at sec 48 with, while the smallest thickness value was detected at sec 29 with the value of $22.6 \mathrm{~mm}$. The difference between the largest value and the lowest value is the total thickness change $(1.91 \mathrm{~mm})$. 


\section{Chapter 6}

\section{Conclusions and Future Works}

In this chapter, conclusions of this thesis study of continuous monitoring of the tissue boundary motion and thickness change due to muscle contraction using a wearable ultrasonic sensor are given. Future works are also discussed.

\subsection{Conclusions}

This research aimed to measure tissues thickness variation quantitatively during muscle contraction using ultrasound. It was very challenging to use a conventional ultrasonic probe for medical imaging due to its limitations (bulky, rigid, and heavy) on this study. However, an ultrasonic sensor developed in our research group is very thin, light, flexible and wearable. Thus, this wearable ultrasonic sensor could overcome the conventional ultrasonic probe limitations. The ultrasonic sensor used in this study was constructed by the PVDF piezoelectric polymer. The total thickness and weight of the sensor were $200 \mu \mathrm{m}$ and $0.3 \mathrm{~g}$, respectively. Its active element size was $15 \mathrm{~mm}$ by $15 \mathrm{~mm}$. Hence, this ultrasonic sensor can be stably attached to a curved and deformable human body surface without restricting the muscle motion underlying the sensor.

However, relatively weak piezoelectric properties and large dielectric/elastic losses of PVDF polymers result in small ultrasonic signal strength. In addition, the echo 
from tissue boundary of two consecutive soft tissues having the similar acoustic impedances had small amplitude. These two factors resulted in the poor SNR of the desired boundary echoes due to noise signals such as scattering (speckle) echoes and random environmental noises. Therefore, signal processing techniques suitable to extract the desired echoes from noise signals needed to be investigated.

Selected signal processing techniques including echo peak detection, crosscorrelation, phase detection, and LQ-factorization were tested by means of numerical simulation experiments to detect tissue boundary echoes to measure tissue thickness variation quantitatively. These signal processing techniques mainly differentiate in two categories: time domain analysis (echo peak detection and the cross-correlation) and frequency domain analysis (phase detection and LQ-factorization). The frequency domain techniques showed better performance than the time domain techniques to detect the boundary echoes and to obtain the boundary displacement. In particular, LQfactorization technique showed the best accuracy among all the techniques tested in the simulation experiments.

In the in-vivo experiment, the phase detection and the LQ-factorization techniques were able to track all of the tissue boundaries. Furthermore, the LQ-factorization technique provided most accurate results among the techniques tested in tracking the boundary motion even with a low SNR signals.

Tissue thickness changes were obtained based on the results from the LQfactorization technique. It was showed that the in-vivo experiment with our experimental configuration showed that that muscle thickness increased during isometric contraction. It 
was also shown that the skin/fat tissue layer thickness decreases during muscle contraction because the muscle layer thickness increased, which squeezed the skin/fat layer.

\subsection{Future Works}

The accuracy of tracking tissue boundary had improved using the signal processing techniques approach as seen in this study. In future work, more signal processing techniques will be considered such as quadrature-phase demodulation, frequencies filters, and adaptive filter.

The electrical and environmental noises should be identified to eliminate them from the measured signals in order to improve the signal processing techniques. It is suggested to search more deeply in the signal components of the boundary echoes and the scattering echoes and to find the differences between them. We are hoping to improve the measurement accuracy of tissue displacement and thickness further during muscle contractions using the wearable ultrasonic sensor, in order to develop medical and clinical applications such as diagnosis of neuromuscular disease, rehabilitation, therapy, and prosthesis control. 


\section{Appendix A}

\section{LQ-factorization Technique Frequency Spectrums}

In the LQ-factorization technique processes as applied in Chapter 5, the center frequency of the boundary echo was found in each boundary echo as follow: from $E_{1}$ $(f c=5.034 \mathrm{MHz})$, from $E_{2}(f c=5.339 \mathrm{MHz})$, from $E_{3}(f c=5.339 \mathrm{MHz})$, and from $E_{4}$ $(f c=4.119 \mathrm{MHz})$ as seen in Figures B.1, B.2, B.3, and B.4. These frequencies are the separated frequencies from the boundary echoes (only one frequency from each boundary echo). Figures B.1, B.2, B.3, and B.4 show the frequency spectrum of: a) the acquired first boundary echo signals, b) the separated signals at the center frequency, and c) the signals without the center frequency. 

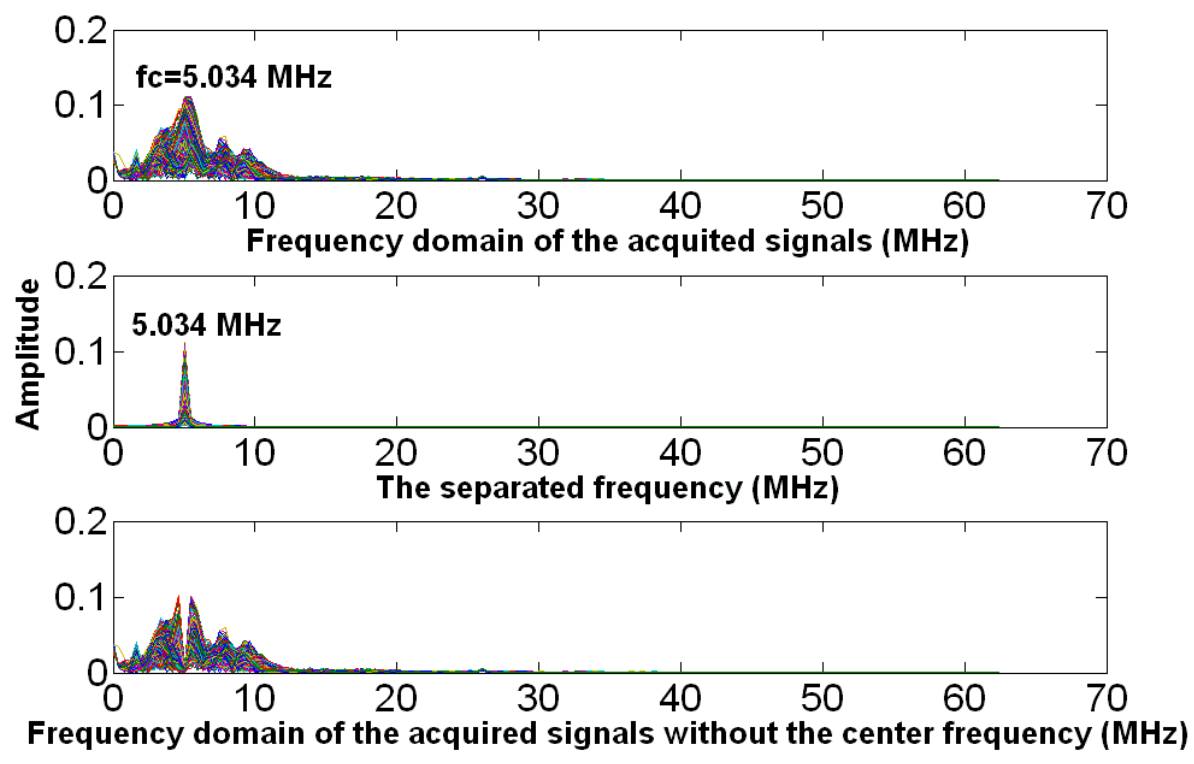

Figure A.1: The frequency spectrum of the $E_{1}$ a) the acquired signals, b) the separated signals at the center frequency, and c) the acquired signals without the center frequency.
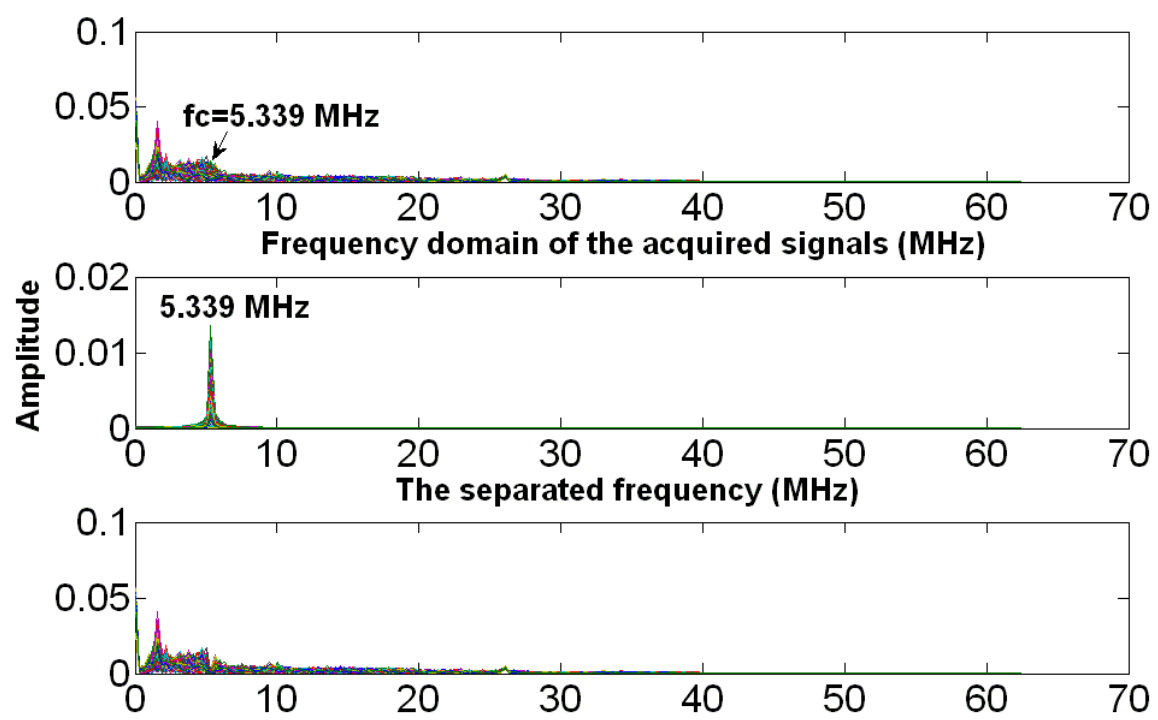

Frequency domain of the acquired singlas withouth the center frequency $(\mathrm{MHz})$

Figure A.2: The frequency spectrum of the $E_{2}$ a) the acquired signals, b) the separated signals at the center frequency, and c) the acquired signals without the center frequency. 

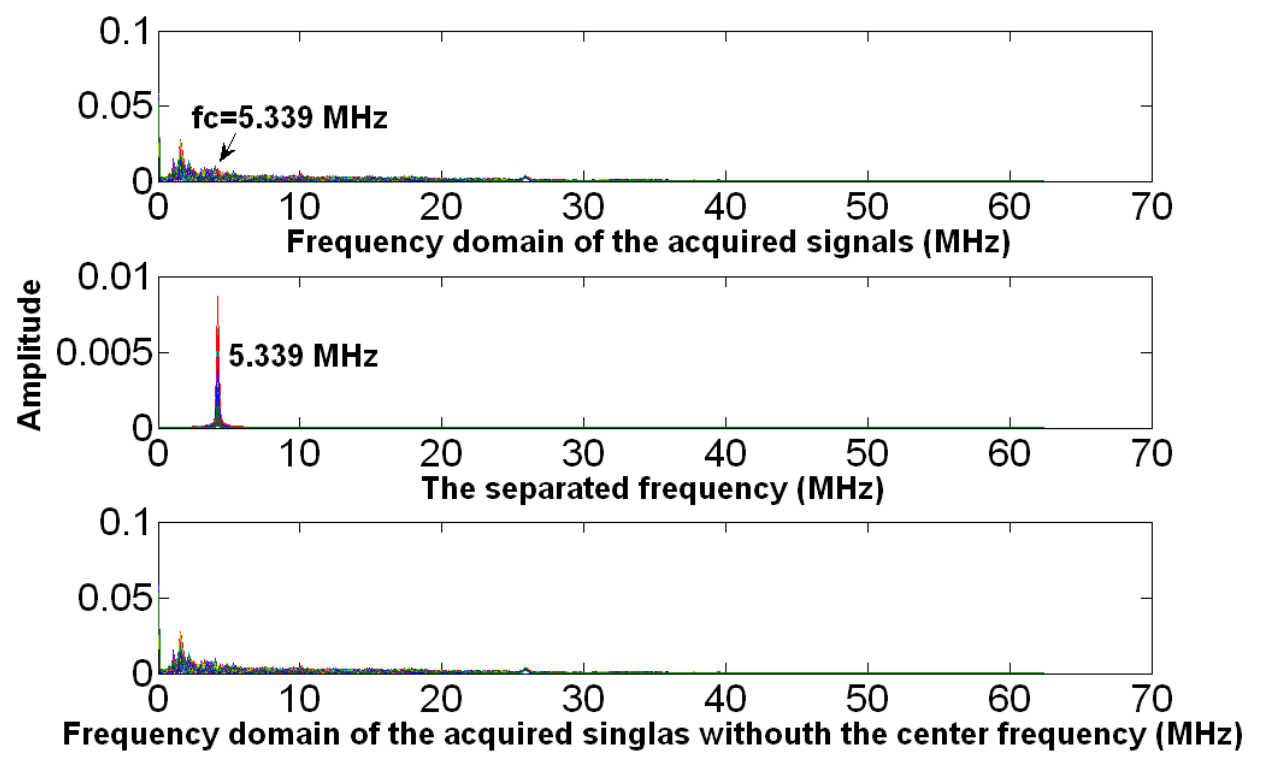

Figure A.3: The frequency spectrum of the $E_{3}$ a) the acquired signals, b) the separated signals at the center frequency, and c) the acquired signals without the center frequency.
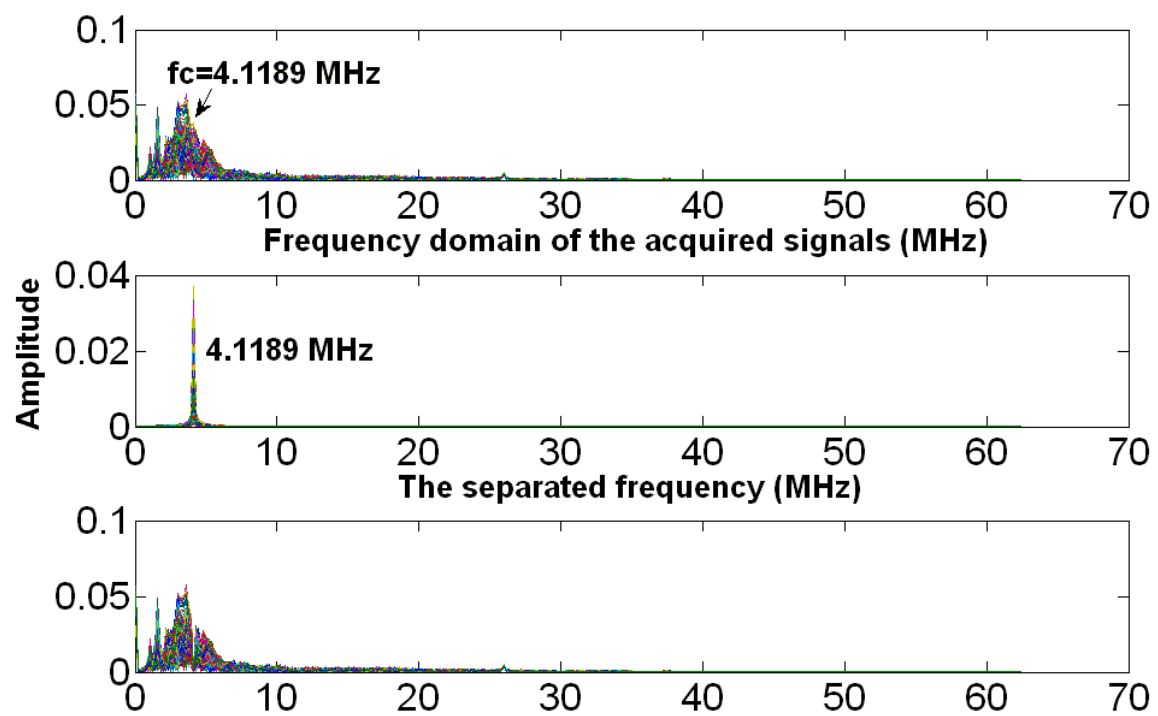

Frequency domain of the acquired singlas withouth the center frequency $(\mathrm{MHz})$

Figure A.4: The frequency spectrum of the $E_{4}$ a) the acquired signals, b) the separated signals at the center frequency, and c) the acquired signals without the center frequency. 


\section{References}

[1] S. Pillen, I.M.P. Arts, and M.J. Zwarts, "Muscle ultrasound in neuromuscular disorders," Muscle \& Nerve, vol. 37, pp. 679-693, 2008.

[2] C.D. Lee, Y. Song, A.C. Peltier, A.A. Jarquin-Valdivia, and P.D. Donofrio, "Ultrasound quantifies the rate of reduction of muscle thickness in amyotrophic lateral sclerosis," Muscle \& nerve, vol. 42, pp. 814-819, 2010.

[3] J.B. Woodhouse and E.G. McNally, "Ultrasound of skeletal muscle injury: An update," Seminars in Ultrasound, CT, and MR, vol. 32, pp. 91-100, 2011.

[4] J. Shi, Y.P. Zheng, X. Chen, and Q.H. Huang, "Assessment of muscle fatigue using sonomyography: Muscle thickness change detected from ultrasound images," Medical Engineering and Physics, vol. 29, pp. 472-479, 2007.

[5] T. Deffieux, J.-L. Gennisson, M. Tanter, and M. Fink, "Ultrafast imaging of in vivo muscle contraction using ultrasound," Appl. Phys. Lett., vol. 89, pp. 184107_1-3, 2006.

[6] J.-W.H. Korstanjea, R.W. Sellesa, H.J. Stama, S.E.R. Hovius, and J.G. Bosch, "Development and validation of ultrasound speckle tracking to quantify tendon displacement," J. Biomechanics, vol. 43, 2010. 
[7] I. AlMohimeed and Y. Ono, "Development of wearable ultrasonic sensor for continuous monitoring of muscle contraction," Proc. Ultrasonic Electronics Symp., vol. 33, pp. 255-256, 2012.

[8] L. F. Brown, "Design considerations for piezoelectric polymer ultrasound transducers," IEEE transactions on ultrasonics, ferroelectrics, and frequency control, vol. 47, pp. 1377-1396, Jan. 2000.

[9] P. Klavora, "Foundations of kinesiology: studying human movement and health," Sport Books Publisher, pp. 77-93, 2007.

[10] D. Pickerell, "Elastography: Imaging of tomorrow?," Journal of Diagnostic Medical Sonography, vol. 26, pp. 109-113, 2010.

[11] M. Linari, I. Doobie, M. Reconditi, N. Koubassova, M. Irving, G. Piazzesi and V. Lombardi, "The stiffness of skeletal muscle in isometric contraction and rigor: the fraction of myosin heads bound to actin," Biophysical Journal, vol. 74, pp. 24592473, 1998.

[12] K. Akataki, K. Mita, and Y. Itoh, "Relationship between mechanomyogram and force during voluntary contraction reinvestigated using spectral decomposition," Eur. J. Appl. Physiol, vol. 80, pp. 173-179, 1999.

[13] C. Orizio and M. Gobbo, "Mechanomyography," Wiley Encyclopedia of Biomedical Engineering, pp. 1-11, 2006. 
[14] C. Orizio, "Surface mechanomyography," in Electromyography: Physiology, Engineering, and Noninvasive Application, R. Merletti and P. A. Parker, Eds., New Jersey: Wiley-IEEE Press, pp. 305-322, 2004.

[15] J. C. Coggshall and G. A. Bekey, "EMG-Force dynamics in human skeletal muscle," Medical and Biological Engineering and Computing, vol. 8, no. 3, pp. 265-270, 2006.

[16] M. A. Mañanas, R. Jané, J. A. Fiz, J. Morera, and P. Caminal, "Study of myographic signal from sternomastoid muscle in patients with chroni obstructive pulmonary disease," IEEE Transactions on Biomedical Engineering, vol. 47, no. 5, pp. 674-681, 2000.

[17] P. K. Kasi, L. S. Krivickas, M. Meister, E. Chew, P. Bonata, M. Schmid, G. Karmen, L. Pu, and E. A. Clancy, "Characterization of motor unit behavior in patients with amyotrophic lateral sclerosis," Proceedings of the 4th International IEEE EMBS Conference on Neural Engineering, pp. 10-13, 2009.

[18] L. Hof, "EMG and muscle force: An introduction," Human Movement Science, vol. 3, pp. 119-153, 1984.

[19] F. Mannion and P. Dolan, "The effects of muscle length and force output on the EMG power spectrum of the erector spinae," Journal of Electromyography \& Kinesiology, vol. 6, pp. 159-168, 1996. 
[20] D. Farina and R. Merletti, "A novel approach for estimating muscle fiber conduction velocity by spatial and temporal filtering of surface EMG signals," IEEE Trans. Biomedical Engineering, vol. 50, pp. 1340-1351, 2003.

[21] M. Pozzo, E. Merlo, D. Farina, G. Antonutto, R. Merletti, and P.E.D. Prampero, "Muscle-fiber conduction velocity estimated from surface EMG signals during explosive dynamic contractions," Muscle Nerve, vol. 29, pp. 823-833, 2004.

[22] J.Y. Hogrel, "Clinical applications of surface electromyography in neuromuscular disorders," Clinical Neurophysiology, vol. 35, pp. 59-71, 2005.

[23] G. ÓLaighin, B. J. Broderick, M. Clarke-Moloney, F. Wallis, and P. A. Grace, "A technique for the computation of lower leg muscle volume from MRI images in the context of venous return," Proceedings of the 29th Annual International Conference of the IEEE EMBS, pp. 961-954, 2007.

[24] B. Juul-Kristensen, F. Bojsen-Møller, E. Holst, and C. Ekdahl, "Comparison of muscle sizes and moment arms of two rotator cuff muscles measured by ultrasonography and magnetic resonance imaging," Eur. J. Ultrasound, vol. 11, pp. 161-173, 2000.

[25] F. Mannion, N. Pulkovski, D. Gubler, M. Gorelick, D. O'Riordan, T. Loupas, P. Schenk, H. Gerber, and H. Sprott, "Muscle thickness changes during abdominal hollowing: an assessment of between-day measurement error in controls and patients with chronic low back pain," Eur. Spine J., vol. 17, pp. 494-501, 2008. 
[26] P. W. Hodges, L. H. M. Pengel, R. D. Herbert, and S. C. Gandevia, "Measurement of muscle contraction with ultrasound imaging," Muscle Nerve, vol. 27, pp. 682-692, 2003.

[27] M. E. Huxley, R. M. Simmons, A. R. Faruqi, M. Kress, J. Bordas and M. H. J. Koch, "Changes in the x-ray reflections from contracting muscle during rapid mechanical transients and their structural implications," J. Mol. Biol, vol. 169, pp. 469-506, 1983.

[28] G. F. Elliott, J. Lowy and B. M. Millman, "Low-angle x-ray diffraction studies of living striated muscle during contraction," J. Mol. Biol, vol. 25, pp. 31-45, 1967.

[29] H. E. Huxley, "Structural difference between resting and rigor muscle; evidence from intensity changing in the low-angle equatorial x-ray diagram," J. Mol. Biol, vol. 37, pp. 507-520, 1968.

[30] H. E. Huxley and W. Brown, "The low-angle x-ray diagram of vertebrate striated muscle and its behaviour during contraction and rigor," J. Mol. Biol, vol. 30, pp. 383434, 1967.

[31] H. E. Huxley, A. Stewart, H. Sosa and T. Irving, "X-ray diffraction measurements of the extensibility of actin and myosin filaments in contracting muscle," Biophysical Journal, vol. 67, pp. 2411-2421, 1994.

[32] J. Kim, Z. Wang, S. B. Heymsfield, R. N. Baumgartner and D. Gallagher, "Totalbody skeletal muscle mass: estimation by a new dual-energy x-ray absorptiometry method," The American Journal of Clinical Nutrition, vol. 76, pp. 378-383, 2002. 
[33] A. Pietrobelli, C. Formica, Z. Wang and S. B. Heymsfield, "Dual-energy x-ray absorptiometry body composition model: review of physical concepts," Am J Physiol Endocrinol Metab, vol. 271, pp. 941-951, 1996.

[34] Z. M. Wang, M. Visser, R. Ma, R. N. Baumgartner, D. Kotler, D. Gallagher and S. B. Heymsdield, "Regional skeletal muscle measurement: evaluation of new dualenergy x-ray absorptiometry model," J Appl Physiol, vol. 87, pp. 1163-1171, 1999.

[35] M. Swash, M. Brown and C. Thakkar, "CT muscle imaging and the clinical assessment of neuromuscular disease," Muscle \& Nerve, vol. 18, pp. 708-714, 1995.

[36] T. Pan, T. Lee, E. Rietzel and G. Chen, "4D-CT imaging of a volume influenced by respiratory motion on multi-slice CT," Medical Physics, vol. 31, pp. 333-340, 2004.

[37] A. Dupont, E. E. Sauerbrei, P. V. Fenton, P. C. Shragge, G. E. Loeb and F. J. Richmond, "Real-time sonography to estimate muscle thickness: Comparison with MRI and CT," Muscle \& Nerve, vol. 29, pp. 230-236, 2001.

[38] D. W. Townsend, T. Beyer and T. M. Blodgett, "PET/CT scanners: A hardware approach to image fusion," Seminars in Nuclear Medicine, vol. 33, pp. 193-204, 2003.

[39] T. Beyer, D. W. Townsend, T. Brun, P. E. Kinahan, M. Charron, R. Roddy, J. Jerin, J. Young, L. Byars and R. Nutt, "A combined PET/CT scanner for clinical oncology," Journal of Nuclear Medicine, vol. 41, pp. 1369-1379, 2000.

[40] D. W. Townsend, "Positron emission tomography/computed tomography," Seminars in Nuclear Medicine, vol. 38, pp. 152-166, 2008. 
[41] J. Gau, Y. Zheng, H. Xie and X. Chen, "Continuous monitoring of electromyography (EMG), mechanomyography (MMG), sonomyography (SMG) and torque output during ramp and step isometric contractions," Medical Engineering \& Physics, vol. 32, pp. 1032-1042, 2010.

[42] C. Lairamore, M. K. Garrison, W. Bandy, and R. Zabel, "Comparison of tibialis anterior muscle electromyography, ankle angle, and velocity when individuals post stroke walk with different orthoses," Prosthet Orthot Int, vol. 35, no. 4, pp. 402-10, 2011.

[43] R. Brunner and J. Romkes, "Abnormal EMG muscle activity during gait in patients without neurological disorders," Gait \&amp; Posture, vol. 27, no. 3, pp. 399-407, 2008.

[44] J. G. A. Cashaback, T. Cluff, and J. R. Potvin, "Muscle fatigue and contraction intensity modulates the complexity of surface electromyography," Journal of Electromyography and Kinesiology, 2012.

[45] Q. Zhou, Y. Chen, C. Ma, and X. Zheng, "Evaluation of upper limb muscle fatigue based on surface electromyography," SCIENCE CHINA Life Sciences, vol. 54, no. 10, pp. 939-944, 2011.

[46] E. Al-Zahrani, C. Gunasekaran, M. Callaghan, P. Gaydecki, D. Benitez, and J. Oldham, "Within-day and between-days reliability of quadriceps isometric muscle fatigue using mechanomyography on healthy subjects," Journal of Electromyography and Kinesiology, vol. 19, no. 4, pp. 695-703, 2009. 
[47] T. W. Beck, T. Housh, A. C. Fry, J. T. Cramer, J. Weir, B. Schilling, M. Falvo, and C. Moore, "MMG-EMG cross spectrum and muscle fiber type," Int J Sports Med, vol. 30, no. 07, pp. 538-544, 2009.

[48] L. Qi, J. M. Wakeling, and M. Ferguson-Pell, "Spectral properties of electromyographic and mechanomyographic signals during dynamic concentric and eccentric contractions of the human biceps brachii muscle," Journal of Electromyography and Kinesiology, vol. 21, no. 6, pp. 1056-1063, 2011.

[49] S. R. Perry-Rana, T. J. Housh, G. O. Johnson, A. J. Bull, J. M. Berning, and J. T. Cramer, "MMG and EMG responses during fatiguing isokinetic muscle contractions at different velocities," Muscle \& Nerve, vol. 26, no. 3, pp. 367-373, 2002.

[50] J.-Y. Guo, Y.-P. Zheng, H.-B. Xie, and X. Chen, "Continuous monitoring of electromyography (EMG), mechanomyography (MMG), sonomyography (SMG) and torque output during ramp and step isometric contractions," Medical Engineering \&amp; Physics, vol. 32, no. 9, pp. 1032-1042, 2010.

[51] E. L. Finanger, B. Russman, S. C. Forbes, W. D. Rooney, G. A. Walter, and K. Vandenborne, "Use of skeletal muscle MRI in diagnosis and monitoring disease progression in duchenne muscular dystrophy," Physical Medicine and Rehabilitation Clinics of North America, vol. 23, no. 1, pp. 1-10, 2012.

[52] A. Magot, F. Caillon, J. M. Mussinia, A. David, and Y. Pereon, "D.P.17 whole body muscle MRI in three patients suffering from XMEA: A peculiar involvement pattern," Neuromuscular Disorders, vol. 22, no. 9-10, p. 823, 2012. 
[53] M. Reconditi, "Recent improvements in small angle x-ray diffraction for the study of muscle physiology," Rep. Prog. Phys., vol. 69, pp. 2709-2759, 2006.

[54] T. Helgason, P. Gargiulo, F. Johannesdottir, P. Ingvarsson, S. Knutsdottir, V. Gudmundsdottir and S. Yngvason, "Monitoring muscle growth and tissue changes induced by electrical stimulation of denervated degenerated muscles with $\mathrm{CT}$ and stereolighographic 3D modeling," Artificial Organs, vol. 69, pp. 440-443, 2005.

[55] P. Gargiulo, T. Helgason, P. J. Reynisson, B. Helgason, H. Kern, W. Mayr P. Ingvarsson and U. Carraro, "Monitoring of muscle and bone recovery in spinal cord injury patients treated with electrical stimulation using three-dimensional imaging and segmentation techniques: Methodological assessment," Artificial Organs, vol. 35, pp. 275-281, 2011.

[56] I. D. Loram, C. N. Maganaris, and M. Lakie, "Use of ultrasound to make noninvasive in vivo measurement of continuous changes in human muscle contractile length," J Appl Physiol, vol. 100, no. 4, pp. 1311-23, 2006.

[57] A. C. Gellhorn, D. C. Morgenroth, and B. Goldstein, "A novel sonographic method of measuring patellar tendon length," Ultrasound in Med. \& Biol, vol. 38, no. 5, pp. 719-726, 2012.

[58] C. Kasai, K. Namekawa, A. Koyano, and R. Omoto, "Real time two-dimensional blood flow imaging using an autocorrelation technique," IEEE Transactions on Sonics and Ultrasonics, vol. SU-32, no. 3, pp. 458-464, 1985. 
[59] T. Loupas, J. T. Powers, and R. W. Gill, "An axial velocity estimator for ultrasound blood flow imaging, based on a full evaluation of the Doppler equation by means of a two-dimensional autocorrelation approach," IEEE Transaction on Ultrasonics, Ferroelectrics and Frequency Control, vol. 42, no. 4, pp. 672-688, 1995.

[60] X. Lai, H. Torp, and K. Kristoffersen, "An extended autocorrelation method for estimation of blood velocity," IEEE Transaction on Ultrasonics, Ferroelectrics and Frequency Control, vol. 44, no. 6, pp. 1332-1342, 1997.

[61] T. Deffieux, J. Gennisson, M. Tanter, and M. Fink, "Ultrafast ultrasonic imaging of in vivo muscle contraction," IEEE Ultrasonics Symposium, pp. 1001-1004, 2006.

[62] J.-Y. Guo, Y.-P. Zheng, Q.-H. Huang, and X. Chen, "Dynamic monitoring of forearm muscles using one-dimensional sonomyography system," Journal of Rehabilitation Research and Development, pp. 187-196, 2008.

[63] M. Rana and J. M. Wakeling, "In-vivo determination of 3D muscle architecture of human muscle using free hand ultrasound," Journal of Biomechanics, vol. 44, pp. 2129-2135, 2011.

[64] J. Shi, Y. P. Zheng, X. Chen, and Q. H. Huang, "Assessment of muscle fatigue using sonomyography: Muscle thickness change detected from ultrasound images," Medical Engineering \& Physics, vol. 29, pp. 472-479, 2007.

[65] J. Shi, J.-Y. Guo, Y.-J. Zhou, and Y.-P. Zheng, "Fatigue-induced continuous changes in muscle pennation angle during isometric contraction," 4th International 
Conference on Bioinformatics and Biomedical Engineering (iCBBE 210), pp. 1-4, 2010.

[66] A. Nordez, T. Gallot, S. Catheline, A. Guével, C. Cornu, and F. Hug, "Electromechanical delay revisited using very high frame rate ultrasound," Journal of Applied Physiology, vol. 106, no. 6, pp. 1970-1975, 2009.

[67] M. N. K. Kristiansen, J. C. Djurhuus, and H. Nygaard, "Design and evaluation of an ultrasound-based bladder volume monitor," Medical and Biological Engineering and Computing, vol. 42, pp. 762-769, Nov. 2004.

[68] C. O'Sullivan, J. Meaney, G.Boyle, J. Gormley, and M. Stokes, "The validity of Rehabilitative Ultrasound Imaging for measurement of trapezius muscle thickness," Manual Therapy, vol. 14, pp. 572-578, 2009.

[69] O. Vasseljen, H. H. Dahl, P. J. Mork, and H. G. Torp, "Muscle activity onset in the lumbar multifidus muscle recorded simultaneously by ultrasound imaging and intramuscular electromyography," Clinical Biomechanics, vol. 21, pp. 905-913, 2006.

[70] S. Brown and S. McGill, "A comparison of ultrasound and electromyography measures of force and activation to examine the mechanics of abdominal wall contraction," Clinical Biomechanics, vol. 25, pp. 115-123, 2010.

[71] S. Sipila and H. Suominen, "Muscle ultrasonography and computed tomography in elderly trained and untrained women," Muscle \& Nerve, vol. 16, pp. 294-300, 1993. 
[72] S. M. Bunce, A. D. Hough and A. P. Moore, "Measurement of abdominal muscle thickness using M-mode ultrasound imaging during functional activities," Manual Therapy, vol. 9, pp. 41-44, 2004.

[73] M. Stokes and A. Young, "Measurement of quadriceps cross-sectional area by ultrasonography: A description of the technique and its applications in physiotherapy," Physiotherapy Practice, vol. 2, pp. 31-36, 1986.

[74] Y. Zheng, M. Chen, J. Shi, X. Chen and Q. Huang, "Sonomyography: Monitoring morphological changes of forearm muscles in actions with the feasibility for the control of powered prosthesis," Medical Engineering \& Physics, vol. 28, pp. 405415, 2006.

[75] T. Koo, C. Wong and Y. Zheng, "Reliability of sonomyography for pectoralis major thickness measurement," J. Manipulative Physiol. Ther., vol. 33, pp. 386-394, 2010.

[76] N. Talbott and D. Witt, "Ultrasound imaging of the serratus anterior muscle at rest and during contraction," Clin. Physiol. Funct. Imaging, vol. 33, pp. 192-200, 2013.

[77] N. R. Grubb, A. Fleming, G. R. Sutherland, and K. A. Fox, "Skeletal muscle contraction in healthy volunteers: assessment with Doppler tissue imaging," Radiology, vol. 194, no. 3, pp. 837-842, 1995.

[78] T. Ikezoe, N. Mori, M. Nakamura and N. Ichihashi, "Age-related muscle atrophy in the lower extremities and daily physical activity in elderly women," Archives of Gerontology and Geriatics, vol. 53, pp. 153-157, 2011. 
[79] M. Rho, T. Spitznagle, L. V. Dillen, V. Maheswari, S. Oza and H. Prather, "Gender differences on ultrasound imaging of lateral abdominal muscle thickness in asymptomatic adults: A pilot study," PM\&R, vol. 5, pp. 374-380, 2013.

[80] L. Ghamkhar, M. Emami, M. A. Mohseni-Bandpei and H. Behtash, "Application of rehabilitative ultrasound in the assessment of low back pain: A literature review," Journal of Bodywork \& Movement Therapies, vol. 15, pp. 465-477, 2011.

[81] C. O'Sullivan, U. M. Persson, C. Blake and M. Stokes, "Rehabilitative ultrasound measurement of trapezius muscle contractile states in people with mild shoulder pain," Manual Therapy, vol. 17, pp. 139-144, 2012.

[82] A. Tieleman, A. Vinke, N. van Alfen, J. van Dijk, S. Pillen and B. van Engelen, "Skeletal muscle involvement in myotonic dystrophy type 2. A comparative muscle ultrasound study," Manual Therapy, vol. 22, pp. 492-499, 2012.

[83] F. Blais and M. Rioux, "Real-time numerical peak detector," Signal Processing, vol. 11, pp. 145-155, 1986.

[84] F. Scholkmann, J. Boss and M. Wolf, "An efficient algorithm for automatic peak detection," Signal Processing, vol. 5, pp. 588-603, 2012.

[85] H. Watzig, "Peak recognition technique by a computer program copying," Chromatographia, vol. 33, pp. 218-224, 1992.

[86] S. M. Tonissen and Y. Bar-Shalom, "Maximum likelihood track-before-detect with fluctuating target amplitude," IEEE Transactions On Aerospace and Electronic Systems, vol. 34, pp. 796-809, 1998. 
[87] T. Amemiya, "Introduction to statistics and econometrics," Harvard University Press Cambridge, pp.1-371, 1993.

[88] N. Sturmel, C, d' Alessandro and F. Rigaud, "Glottal closure instant detection using lines of maximum amplitudes LOMA," ICASSP IEEE, pp.4517-4520, 2009.

[89] J. Stewart, "Calculus: Early transcendental," Brooks/Cole Cengage Learning, 2012.

[90] S. Klimenko, G. Mitselmakher and A. Sazonov, "A cross-correlation technique in wavelet domain for detection of stochastic gravitational waves," University of Florida, pp. 1-15, 2002.

[91] J. Hansen, "Selected approaches to estimation of signal phase," CiteSeer, pp. 1-15, 2003.

[92] J. A. Putman, "Signal processing tTechniques," EEG Info., pp. 1-16, 1989.

[93] J. W. Cooley and J. W. Tukey, "An algorithm for the machine calculation of complex fourier series," American Mathematical Society, pp. 297-301, 1989.

[94] B. V. Dun and M. Moonen, "Multi-channel wiener filtering based auditory steadystate response detection," ICASSO IEEE, vol. 2, pp. 929-932, 2007.

[95] B. V. Dun, J. Wouters and M. Moonen, "Improving auditory steady-state response detection using independent component analysis on multichannel EEG data," Biomedical Engineering IEEE Transactions, vol. 54, pp. 1220-1230, 2007.

[96] A. H. Sayed, "Adaptive filters," John Wiley \& Sons, pp. 12-24, 2011. 
[97] J. L. Prince and J. M. Links, "Medical imaging signals and systems," Pearson Prentice Hall, 2006.

[98] Y. Shmaliy, "Continuous-time signals," Springer, pp. 241-249, 2006.

[99] M. B. Rhudy, "Real time implementation of a military impulse noise classifier," Pennsylvania State University, 2006.

[100] R. Demirli and J. Saniie, "Model-based estimation of ultrasonic echoes. Part I: Analysis and algorithms," Ultrasonics, Ferroelectrics and Frequency Control, IEEE Transactions on, vol. 48, no. 3, pp. 787-802, 2001.

[101] Q. Zhang, G. Yang, and P. Que, "Ultrasonic signals processing base on parameters estimation," Russian Journal of Nondestructive Testing, vol. 45, no. 1, pp. 61-66, 2009.

[102] R. J. Hyndman, and A. B. Koehler, "Another look at measures of forecast accuracy," International Journal of Forecasting, vol. 22, pp. 679-688, 2006.

[103] J. S. Armstrong and F. Collopy, "Error measures for generalizing about forecasting method: Empirical comparisons," International Journal of Forecasting, vol. 8, pp. 69-80, 1992.

[104] R. C. Chivers and R. J. Parry, "Ultrasonic velocity and attenuation in mammalian tissues," J. Acoust. Soc. Am., vol. 63, pp. 940-953, 1978.

[105] T. Koch, S. Lakshmanan, S. Brand, M. Wicke, K. Raum and D. Morlein, "Ultrasound velocity and attenuation of porcine soft tissues with respect to structure and composition: I. Muscle," Meat Science, vol. 88, pp. 51-58, 2011. 
[106] J. O’Neill, "The forearm, musculoskeletal ultrasound," Springer, pp. 103-109, 2008.

[107] F. H. Netter, "Atlas of human anatomy," Saunders Elsevier, 1998.

[108] A. Arthur, F. Dalley, and K. L. Moore, "Clinically oriented anatomy," Lippincott Williams \& Wilkins, a Wolters Kluwer Business, 1980.

[109] G. J. Tortoa and B. Derrickson, "Principles of anatomy \& physiology," John Wiley \& Sons Inc., Wolters Kluwer Business, 2012.

[110] E. N. Marieb and K. Hoehn, "Human anatomy and physiology," Pearson, 2007.

[111] I. AlMohameed, "Development of wearable ultrasonic sensors for monitoring muscle contraction," MASc thesis in Biomedical Engineering, Carleton University, 2013. 